(C) [2012]

Jo Ann F. Cummings

ALL RIGHTS RESERVED 


\title{
AN ETHNOGRAPHY OF THE CULTURE OF PAIN IN A NON-PEDIATRIC EMERGENCY DEPARTMENT
}

\author{
By \\ JO ANN F. CUMMINGS \\ A Dissertation submitted to the \\ Graduate School-Newark \\ Rutgers, The State University of New Jersey \\ In partial fulfillment of the requirements \\ For the degree of \\ Doctor of Philosophy \\ Graduate Program in Nursing \\ Written under the direction of \\ Elise L. Lev \\ and approved by \\ Newark, New Jersey
}

May 2012 


\begin{abstract}
OF THE DISSERTATION
An Ethnography of the Culture of Pain in a Non-Pediatric Emergency Department By JO ANN F. CUMMINGS

Dissertation Director:

Elise L. Lev

Rationale for the Study: Despite the exponential growth in pharmacological and nonpharmacological methods for the treatment of pediatric procedural pain there remains a gap between available interventions and actual practice in the emergency department clinical setting. Researchers have recommended the examination of unit culture since culture is proposed to be a major factor that affects the implementation of pain management practices.
\end{abstract}

Method: Ethnography was employed to gain access to the beliefs and practices of healthcare participants in a Northeastern non-pediatric emergency department. Data were collected for 5 months with over 100 hours of observation. Six key informants were interviewed and 44 pediatric procedural interactions with 27 healthcare providers during the treatment of children two to eight years of age undergoing procedures were observed. Other information gathered included documents from the institution, and pain related information from the patient's medical record. All interviews were recorded and transcribed verbatim. Data were managed using the Ethnograph 6.0 and analyzed using the procedure developed by Leininger and McFarland (2006).

Results: Seven major themes with categories were discovered that included assessment, 
procedural pain, treatment of pain, communication related to comfort, fragmentation of care in the emergency department, healthcare provider attitudes, and environmental issues and policies.

Conclusion: The results of this study suggest that practice environment of the emergency department and organizational culture exerted a significant degree of influence over healthcare providers' pediatric pain management practices. Nurses' did not use pain measurement tools as they were intended to be used and instead improvised measurement by observing the child's behavior. The management of children during painful procedures consisted of an array of behaviors and practices. The use of physical restraint during painful procedures was a common practice. Participants continue to hold many assumptions and misconceptions about pediatric pain assessment and management. The findings of this study provide insight to the everyday practice of emergency department healthcare providers for pediatric pain in a non-pediatric setting. 


\section{Acknowledgments and Dedication}

I would like to thank my family who helped to watch over my children and gave me peace of mind, especially during the long hours of field work. Maura and Aidan, my children, whose patience and understanding have been deeply appreciated. I am grateful to my husband Joseph for his constant loving support and encouragement through this academic journey. Thanks are also due to my colleagues; Annemarie DowlingCastronovo and Tresa Dusaj whose discussions throughout the research process help navigate me stay on course. I owe a debt of gratitude to my dissertation chair Elise Lev, and committee members Mary Ann Scoloveno, Ganga Mahat, and Geri Dickson for their guidance and mentorship. Lastly, thank you to all my professors in the graduate faculty at the College of Nursing who challenged my intellect and shaped me into the person I am today.

This work is dedicated to my grandmother Rufina who taught me the value of an education and to always reach for the stars. 


\section{Table of Contents}

Notice of Copyright

Title page

Abstract $\quad$ ii

Acknowledgement and Dedication $\quad$ iv

Table of Contents $\quad$ V

List of Tables

Chapter I. Introduction and Theoretical Perspective 1

Chapter II. Literature Review 12

$\begin{array}{ll}\text { Chapter III. Methods } & 37\end{array}$

Chapter IV. Contexts and Informants $\quad 54$

$\begin{array}{ll}\text { Chapter V. Description and Discussion of Themes } & 67\end{array}$

Chapter VI. Discussion of Findings 121

$\begin{array}{ll}\text { Chapter VII. Conclusion } & 162\end{array}$

$\begin{array}{ll}\text { References/Bibliography } & 173\end{array}$

Appendix A: IRB Approval Form 209

Appendix B: Key Informant Demographics 213

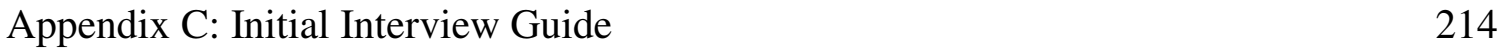

$\begin{array}{ll}\text { Appendix D: IRB Approval Form } & 215\end{array}$

Appendix E: Informed Consent Forms 217

Appendix F: Table 1: General Age Distribution of Emergency Patients 219

$\begin{array}{ll}\text { Appendix G: Code Case Study } & 220\end{array}$ 
Appendix H: Table 2: Communication Behaviors

Appendix I: Table 3: Demographic Data Key Informants

Appendix J: Final Interview Guide

Appendix K: Audio Consent Form

Appendix L: Memo Emergency Department

Appendix M: Memo Reflective and Analytic

Appendix O: Restraint Policy

Appendix P: Hospital Closure New Jersey

Appendix Q: Management of Patient with Pain General Hospital Policy 


\section{List of Tables}

Table 1: General Age Distribution of Emergency Department Patients 219

Table 2: Communication Behaviors 223

Table 3: Demographic Data Key Informants 224 


\section{Chapter I}

It was always Stewart on the table and not us. This procedure had my son surrounded by the strong arms of nurses in the great lock and shout down, straight from the eighteenth century. Maybe our senses were heightened, to hear little Stewart ever so faintly, screaming through the thick wall that separated his mother and me from him. We suffered with him and wondered why, in the year of our Lord 1992, Stewart had to be awake for this. (Baxter,1993, p.1018).

The above excerpt came from a letter sent to the editor of a pediatric journal. Baxter was the father of a 4-year-old child who had underwent a painful procedure. Over a decade has passed since the initial letter was written, and despite the exponential growth in the treatment options for pediatric procedural pain, standard clinical practice in the emergency setting continues to inadequately address prevention and treatment of procedural pain in children (American Academy of Pediatrics \& American Pain Society, 2001; Bhargava \& Young, 2007).

For the past four decades researchers in the area of pain management have contributed to the extensive literature which includes issues on patient characteristics, pain measurement, efficacy of treatments, physiology, and psychosocial issues. An area which has gained more attention by clinicians and researchers is that of procedural pain. As a category of acute pain, children have identified diagnostic and monitoring procedures as the most feared and painful of events (Cummings, Reid, Finley, McGrath, \& Ritchie, 1996; Hester, 1995; Kortesluoma \& Nikkonen, 2004; Liossi, 2002). The most common source of procedural pain has been documented to be needle pain (Blount, Piira, \& Cohen, 2003; Cohen, 2002; Rogers \& Ostrow, 2004; Uman, Chambers, McGrath, \& Kisley, 2006).

During 2005, pediatric patients represented $21 \%$ of emergency department visits in the United States (Nawar, Niska, \& Xu, 2007). Studies have continuously shown pain 
management to be deficient in pediatric patients treated in the emergency department setting (Alexander \& Manno, 2003; Bhargava \& Young, 2007; Brown, Klein, Lewis, Johnston, \& Cummings, 2003; Cimpello, Khine, \& Avner, 2004; Cordell, et al., 2002; Drendel, Brousseau, \& Gorelick, 2006; MacLean, Obispo, \& Young, 2007; Probst, Lyons, Leonard, \& Esposito, 2005; Rupp \& Delaney, 2004). For example, Bhargava and Young (2007) documented only $38 \%$ of pediatric patients were provided with some form of pharmacological pain management for intravenous catheter placement, and MacLean, Obispo, and Young (2007) found that less than $1 \%$ of patients received pain management for venipuncture or intravenous line placement.

\section{Concern to be Addressed}

The advances of treatment options for pediatric procedural pain continue to be underutilized by healthcare providers in the emergency department unit. While there are a multitude of research based evidence that has examined the efficacy of using both pharmacological (Cregin et. al., 2008; D’Arcy, 2007; Meunier-Sham, \& Ryan, 2003) and non-pharmacological (Carlson, Broome, \& Vessey, 2000; D’Arcy, 2007; Lassetter, 2006) interventions, there remains a gap between available pediatric procedural pain management and actual practice in the emergency department clinical setting (MacLean et al., 2007; Ramponi, 2009).

When pediatric pain is inadequately treated, it can lead to physical and psychological consequences (American Academy of Pediatrics \& American Pain Society, 2001; Agency for Health Care Policy and Research, 1992). Multiple researchers have documented both the short-term and long-term effects from inadequately treated pediatric pain, especially in younger children (Blount et al., 2003; Schechter, Berde, \& Yaster, 
2003). Short-term consequences to the patient include suffering during the procedure, increased distress, hypoxemia, and altered metabolic stress response (Pokela, 1994; Young, 2005). Consequences for the healthcare provider include prolongation of the procedure (Cohen, Bernard, Greco, \& McClellan, 2002), the assistance of more staff during the procedure, and the potential for more than one attempt due to initial failure. Long term consequences include mortality (Demir, 2007a; Reynolds, 2001), avoidance of medical care during young adulthood, exaggerated pain response and behaviors during future procedures (Garg, Narsinghani, Bhutta, Rovnaghi, \& Anand, 2003; Hermann, Hohmeister, Demirakca, Zohsel, \& Flor, 2006; Pate, Blount, Cohen, \& Smith, 1996; Schechter et al., 2003).

Evidence suggests that early painful stimuli might permanently alter the neuronal circuits that process pain in the spinal cord. For example, Melzack (2001) proposed in his neuromatrix theory of pain that pain is a multidimensional experience produced by characteristic neurosignature patterns of nerve impulses generated by a widely distributed neural network. The neurosignature output pattern is determined by multiple influences that converge on the neuromatrix. The neurosignature pattern is modulated in part by sensory inputs but also by cognitive events, such as psychological distress (Melzack, 2001).

\section{Phenomenon of Concern}

The phenomenon of concern in this study is to discover the role of unit culture on pediatric procedural pain management in a non-pediatric emergency department. For this study, culture is defined as the acquired knowledge and shared meaning healthcare providers use to interpret experience and generate behavior (Spradley \& McCurdy,1972; 
Spradley, 1980 ) as they go about their assessment and management of pediatric procedural pain.

In general, emergency departments are viewed as chaotic and frightening for young children. Healthcare workers may justify holding the child down and performing the procedure as the most compassionate alternative (Read, 1994; Young, 2005). Younger children especially those aged 1 to 7 -years of age are considered vulnerable. Researchers have purported that the gold standard of pain measurement is the child's self-report of pain (Broome \& Huth, 2003); however, self-report instruments are appropriate for children who are able to complete simple seriation tasks or conceptualize things in order of magnitude (Hunt, 2006). The young child's limited ability to communicate pain requires the healthcare professional to use behavioral observation; therefore, the healthcare providers assumptions related to the meaning of the behavior should be carefully examined (American Academy of Pediatrics \& American Pain Society, 2001). An exploration of unit culture in a non-pediatric emergency department may provide some insight into the less than optimal treatment of pain in the young pediatric patient.

\section{Purpose}

The purpose of this research is to describe the unit culture of the non-pediatric emergency department, explore the ways healthcare providers evaluate and treat pediatric procedural pain and explore attitudes of healthcare providers towards treating pediatric procedural pain. Little is known about how pediatric procedural pain is managed by healthcare providers in a non-pediatric emergency department unit. The lack of change in pain management for the pediatric population indicates the importance of conducting research in the clinical practice setting (Ely, 2001). Researchers have recommended that 
further investigations examine the culture of the unit where care is provided since culture is assumed to be a major factor that affects the implementation of pediatric procedural pain management practices (Alley, 2001; Ely, 2001; Foster, 1991; Hemmelgarn, Glisson, \& Dukes, 2001; Idell, Grant, \& Kirk, 2007). Improvement in pain management within healthcare requires a focus on systems of care, including the establishment of processes to support, reinforce, and reward appropriate and effective pain management (Gordon et al., 2002). Therefore, the use of a qualitative approach and focused ethnography is the most appropriate choice to addressing the purposes of this study. The main research question is formulated as: What social and cultural factors influence healthcare providers' evaluation and treatment of pediatric procedural pain in a non-pediatric emergency department?

\section{Guiding Questions}

The study was guided by the following questions:

1. How are healthcare providers pediatric procedural pain management behaviors organized within the non-pediatric emergency department?

2. How is pediatric procedural pain management perceived by healthcare providers in the non-pediatric emergency department?

3. What are the unwritten rules for managing pediatric procedural pain in the nonpediatric emergency setting?

4. How are these unwritten rules for managing pediatric procedural pain in the nonpediatric emergency setting perceived by members of the emergency staff?

5. What are the pediatric procedural pain management practices of emergency staff in a non-pediatric emergency department? 
6. What are the beliefs regarding pediatric procedural pain management of emergency staff in the non-pediatric emergency department?

7. What tools are in place to assist the emergency staff in managing pediatric procedural pain in the non-pediatric emergency setting?

\section{Foundational Assumptions}

The first assumption of the researcher is that social factors represent defining features of human environments and are powerful sources of variation (Craig \& Pillai Riddell, 2003) in how emergency departments operate.

The second assumption of the researcher is that emergency staff will be focused on carrying out the technical aspects of children's care, and comforting children will not be a major activity (Byrne, Morton, \& Salmon, 2001).

The third assumption of the researcher is that pain assessment of preoperational children are based on nurses' intuition of what the child is experiencing without the use of any standardized method of interpreting and quantifying pain behaviors (Manworren \& Hynan, 2003).

The fourth assumption of the researcher is that simple non-pharmacological methods such as distraction will not be used with any regularity. Researchers have reported that despite nurses stating that they used non-pharmacological methods to relieve children's pain, the implementation of non-pharmacological methods remains low (Twycross, 2007a; Woodgate \& Kristjanson, 1996a).

The fifth assumption of the researcher is that healthcare providers in the nonpediatric emergency department will have a negative and non-supportive attitude towards pediatric procedural pain management. The impact of attitudes, beliefs, and knowledge 
on the effectiveness of pain management, and the under treatment of patients by healthcare providers has been well documented (Comely \& Banks, 2000; Hennes, Kim, \& Pirrallo; 2005; Pack-Mabien, Labbe, Herbert, \& Haynes, 2001).

The sixth and final assumption of the researcher is that human behavior is shaped in the context of a sociocultural milieu and that every human event is culture-bound (Shimahara, 1984).

\section{Definition of Terms}

Culture. Culture is defined as the acquired knowledge and values healthcare providers use to interpret experience and generate behavior (Spradley \& McCurdy, 1972; Spradley, 1980). It consists of the shared meanings that guide principles of action and organization with regard to pediatric procedural pain management, including values, beliefs, and rules of conduct (Wolcott, 2008).

Non-Pediatric Emergency Department. Non-pediatric emergency department is a unit located within the hospital which is specifically equipped and staffed to evaluate, treat, and provide rapid care to individuals from birth to geriatrics with sudden and acute illnesses or trauma (Smith \& Feied, 1999), without a specific pediatric focus.

Non-Pharmacological Therapy. Non-pharmacological therapies are considered nonallopathic interventions used in a holistic approach to promote the physical and psychological comfort of children in pain (Lassetter, 2006).

Pre-operational Stage. The pre-operational stage is based on Piaget's cognitive theory of development and includes children from 2 to 7 -years of age (Lowenthal, 1978). In this phase, children have a limited sense of seriation and therefore have difficulty 
conceptualizing things in order of magnitude. This is an important aspect as most selfreport scales are based on the concept of seriation (Hunt, 2006).

Procedural Pain. Procedural pain is an unpleasant sensory and emotional experience (International Association for the Study of Pain, 1979) which is the result of routine medical and nursing procedures used to obtain diagnostic information and/or perform therapeutic interventions in the emergency department.

\section{Significance}

\section{Critical Problems}

This study is significant for nursing as pain management is one of the 433 interventions identified as a critical nursing function in The Nurse Intervention Classification System (McCloskey \& Bulechek, 1996). The increase use of health care technology by nurses in the emergency department such as intravenous cannulation, venipuncture, and nasogastric tube insertion provides a rationale for addressing this clinical problem. The increased demands on nurses to be flexible and avoid unfavorable cultural imposition practices may contribute to the suffering of children and their under treatment of pain (Leininger, 1995). For example, if a nurse's view of pain expression is not consistent with a child's view of pain then the likelihood is that care decisions and actions may not be initiated to alleviate that child's pain (Leininger, 1995).

\section{Clinical Practice}

Pain management has traditionally been a nursing responsibility (Ludwig-Beymer, 2008). The management of pain is important to nursing because nurses in the context of their practice frequently encounter people either experiencing or anticipating pain (Ludwig-Beymer, 2008; Villarruel, 1995). Nurses have the most frequent and closest 
contact with children presenting for non-emergency illness and injury care and spend more time with patients in pain than any other healthcare professional (Carlson et al., 2000).

\section{New Insights}

Culture has long been recognized in nursing practice and research as a factor that influences a groups' response to pain (Ludwig-Beymer, 2008). Based on this premise an ethnographic study may reveal new insights about pediatric procedural pain management practices within a non-pediatric emergency unit. Ethnography is not only a method of data analysis, but also a way of conceptualizing and looking at a particular group or culture (Fetterman, 1998; Spradley \& McCurdy, 1972; Spradley, 1980; Wolcott, 2008). A focused ethnography will be structured around a distinct problem pediatric procedural pain management practices, and the problem is examined within a specific context; the non-pediatric emergency unit (Roper \& Shapira, 2000).

\section{The Researcher's Perspective}

I am a Board Certified Emergency Nurse since 1997. For the first six years of my practice I worked at the bedside in various suburban non-pediatric emergency departments. During my time in the emergency department I assisted with many medical procedures on children such as suturing, fracture reduction, and lumbar puncture while also conducting various nursing procedures such as intravenous cannulation, venipuncture, intramuscular injections, nasogastric tube insertions, and uretheral catheterizations. Although I strove to address the pain needs of children, I often felt frustrated by the lack of attention and marginalization given to the pain experienced by children. 
After finishing my graduate studies as a pediatric nurse practitioner, I realized that there are a multitude of pharmacological and non-pharmacological interventions available to assist healthcare providers. It has been eight years since I last worked at the bedside in an emergency department, but I continue to hear stories from both families and nurses about the under treatment of children's procedural pain. My question is, why with the advances in pain management technology are children still not treated for procedural pain in the emergency department setting?

As an emergency nurse, I recognize that I have my personal views pertaining to the research question. Ethnographic researchers must first make specific biases explicit (Fetterman, 1998; Roper \& Shapira, 2000). An ethnographer begins with biases and preconceived notions about how people behave and what they think, when controlled biases can focus and limit the research effort (Fetterman, 1998). The use of self in ethnographic research dictates a high degree of reflexivity. Reflexivity refers to researchers being part of, rather than separated from the data and using self-awareness as a source of insight (Aamodt, 1982; Lipson, 1991). My decision to conduct this study is derived from both my personal experiences as an emergency department nurse and my professional concerns.

\section{Summary}

Despite the advances of treatment options for pediatric procedural pain the implementation of those interventions remains underutilized by healthcare providers in the emergency department (ED) setting. This study will describe the unit culture of the non-pediatric ED, explore the ways healthcare providers evaluate and treat pediatric procedural pain, and explore attitudes of healthcare providers towards treating pediatric 
procedural pain. The findings of this study will provide a picture of how providers in the non-pediatric ED setting assess and manage pediatric pain. Understanding healthcare provider views, and the manner that care is delivered may be useful to those professionals in the organization planning to design and implement change for improved pediatric pain management practices. 


\section{Chapter II}

\section{Literature Review}

In qualitative research, review of the literature is used to demonstrate knowledge of previous work in the area, provide the basis for research, and the context for interpreting findings (Meadows, 2003; Ryan, Coughlan, \& Cronin, 2007). According to Sandelowski and Barroso (2002) the literature review may show contradiction logic where writers emphasize the inconsistencies in knowledge and point to a purpose that will help to resolve this contradiction. The research literature on pediatric pain management is extensive. The intent of this literature review is to situate the study, to establish gaps in knowledge of the phenomenon, provide a foundation for the significance of using an ethnographic approach, and to formulate study questions. This literature review will be expanded as appropriate throughout the data analysis process.

\section{Historical Perspective}

Pain management was brought to the forefront of healthcare in the 1990's. As a result of the under treatment of pain and the variability of treatments, the Agency for Health Care Policy and Research (AHCPR, 1992) developed the first comprehensive clinical practice guidelines for acute pain management (Jacox, Ferrell, Heidrich, Hester \& Miaskowski, 1992). In late 2000, Congress passed into law a provision declaring the tenyear period that began January 2001, as the Decade of Pain Control and Research (Hamdy, 2001). The following year, in January 2001, the Joint Commission on Accreditation of Healthcare Organization (JCAHO, 1999) released new standards for pain. The performance standards of pain have been emphasized by referring to it as the fifth vital sign (Polomano, Dunwoody, Krenzischek, \& Rathmell, 2008). The importance 
of pain in patient outcomes has led to standards that require the assessment, reassessment, and documentation of pain on a regular basis, and evaluation of pain following interventions to manage pain (Phillips \& JCAHO, 2000; Polomano et al., 2008; Stumbo, 2002).

Researchers at the World Health Organization noted there was a lack of priority given for pain management during interventional procedures in very young children, recognized pain as a problem in its own right not just an indicator of underlying disease or injury, and proper treatment of pain as an important public health concern (Kumar, 2007). Critical times when patients were most likely to experience gaps in analgesia have been reported to be during and after painful procedures (Polomano et al., 2008).

\section{Demographics}

In the United States, the vast majority of children are cared for in non-pediatric hospital emergency departments, emergency departments that are located in general hospitals rather than in hospitals dedicated to the care of children (American Academy of Pediatrics, Committee on Pediatric Emergency Medicine, 2007; Gausche-Hill, Schmitz, \& Lewis, 2007).

In 2007, a survey which assessed the degree of pediatric preparedness of emergency departments in the United States showed that $89 \%$ of pediatric visits occurred in nonchildren's hospitals, $26 \%$ of visits occurred in rural or remote facilities and $75 \%$ of emergency departments saw less than 7000 children per year (Gausche-Hill et al., 2007). Researchers have concluded that because pediatric patients' represent the minority age group in general emergency departments, emergency department staff may lack familiarity with pediatric emergencies, lack sufficient opportunities to regularly practice 
the cognitive and technical skills necessary for providing emergency pediatric care (American Academy of Pediatrics, Committee on Pediatric Emergency Medicine, 2007; Gausche-Hill et al., 2007; Probst, Lyons et al., 2005).

A major area of focus over the past two decades has been the identification of factors affecting pain assessment and management. A review of the empirical research on the barriers affecting pain management have been categorized by the Agency for Health Care Policy and Research (1992) to include healthcare system related, healthcare provider related, and patient related areas. To gain knowledge of cultural meanings of pain management practices by clinicians' in the emergency department, the review of the literature is organized around the foundational assumptions mentioned in chapter one which will include sociocultural and environmental contexts of the phenomenon being studied.

\section{Social Influence}

In general, adaptation to social factors represents a significant source in emergency department differences of how pediatric procedural pain is addressed. Agents of socialization include the physicians, nurses, and allied health personnel. The healthcare provider learns how to respond to a painful procedure indirectly by observing and imitating behaviors of others more experienced in the unit, and the approval or disapproval of a specific behavior will determine the specific behavior's maintenance or extinction (Abu-Saad, 1984). For example, research studies with surgical and pediatric emergency nurses reported although nurses' were in a position to determine the best methods of pain reduction for patients, the implementation of pain free strategies was thwarted by the inability to obtain physician order (Meunier-Sham \& Ryan, 2003; 
Woodgate \& Kristjanson, 1996a). This process has indicated changes to new or novice nurses attitudes towards care itself. Parts of this process appeared to be largely negative, with a reduction in the importance of care, the adoption of personally cynical attitudes and willingness to make allowances for environmental and circumstantial constraints (Mackintosh, 2006).

Collegial consultation and support are an essential component of the unit milieu and influence nurses' pain management practices (Broome \& Huth, 2003). Lack of communication between nurses' and physicians' was identified as a barrier to effective pain management practices (Allcock, McGarry, \& Elkan, 2002; Kaasalainen, et al., 2007). The nurse-physician collaboration is especially fundamental to the pharmacological treatment of pain. Schmidt and Svarsdtad (2002) reported a positive correlation between the quality of pharmacological management and the quality of nursephysician collaboration. In a survey of 1,015 registered nurses examining the barriers to pain management resulted in $63.6 \%$ identified poor cooperation by physicians in relation to nurses' suggestion, $69.9 \%$ identified inadequate prescription of analgesic medications, and $66 \%$ related physicians' knowledge and perceptions of pain as barriers (Van Niekerk \& Martin, 2003).

Researchers have supported the argument that the values, attitudes, and skills that nurses learn as they are socialized into the nursing culture serve to impede attempts to improve patient handling techniques (Kneafsey, 2000). For example, researchers have reported that nurses adapt their behavior to conform to the prevailing ward culture (Kneafsey, 2000; Mooney, 2007; Philpin, 1999), so that if it is not common practice on a 
unit to use pain assessment tools, even nurses who know how pain should be managed may not do so (Twycross, 2007a).

Taylor (1997) utilized participant observation and interviews to examine the cognitive processes involved in carrying out nursing procedures. The results revealed that novices were not problem solving but copying a role model's previous performance. The researcher concluded the determining influence of novice behavior was the desire to become a member of the unit by carrying out procedures in a like manner. This finding was supported by Twycross (2007b) who explored the relationship between nurses' theoretical knowledge and the quality of their practices by using a pain management checklist reflective of current best practice pain management guidelines against observed practice. Results revealed that nurses' appeared to copy the behaviors of more senior staff. The quality of a nurse's practice was dependent on the practices of the role-model. If a role-model did not assess pain or use non-drug methods of pain-relief the novice nurse adopted a similar approach. The research findings discussed support that even when a nurse has a good level of theoretical knowledge or considers a pain management task important, it is unlikely to be applied in practice (Twycross, 2007b, 2008).

Research findings have supported the need for new staff to fit in, comply with the rules, and follow the routines of the unit (Wilson, McCormack, \& Ives, 2005). Teamwork is fundamental to the smooth operation of the emergency department. All healthcare providers essentially work as one unit and it is this cohesion that creates a feeling of camaraderie and friendship between them. This comfortable atmosphere allows for easy communication on the job and in swapping shifts, patients and or tasks (Wolf, Evans, Hewitson, \& Perez, 2003). The lack of adoption to unit norms leads to lack of cohesion, 
inconsistency in working relationships, no pathway for bringing one's own ideas or ways of working and tensions to challenging decisions of staff (Dalton, et al., 2001; Mooney, 2007; Wilson et al., 2005).

Some researchers have concluded that the socialization process of new or novice nurses to practice is ritualistic and non-patient-focused (Mooney, 2007). Failure to adopt behaviors has been documented to lead to negative sanctions which are used to ensure role learning such as being yelled at in front of peers, picked on, and excluded if nurses failed to conform to the area's norms (Philpin, 1999).

\section{Technical Aspects of Care}

Researchers have found that increased specialization and technology have decreased the more nurturing aspects of nursing (Byrne \& Heyman, 1997; Carter et al., 2008; Woodgate \& Kristjanson, 1996a,1996b), and the work culture of most settings are organized on a task oriented basis (Carter, et al., 2008; Philpin, 1999). The increased demand of technology continues to diminish the independent role of nursing removing the priority of providing comfort, support, and education to patients and families and placing priority on checking orders, starting intravenous lines, mixing medications, and monitoring equipment (Brown, 1992). This conjecture is supported by the results of various studies (Byrne \& Heyman, 1997; Carter, et al., 2008; Chambliss, 1996; Fisher \& Peterson, 1993; Olive, 2003; Philpin, 1999; Woodgate \& Kristjanson, 1996a, 1996b) which reported that nurses tend to focus on skills and tasks while minimizing the nursepatient interaction. Results from qualitative studies that explored experiences and values of nurses reported that unit emphasis on technological interventions inhibited nurses' ability to provide the holistic, individual care advocated in current nursing theory (Olive, 
2003; Philpin, 1999). The totality of patient care was broken down into specific tasks performed by a number of different nurses and support workers (Olive, 2003; Philpin, 1999).

The completion of work and accomplishment of tasks remains a powerful underpinning of work culture in more work settings (Carter, et al., 2008). Nurses seem to focus on tasks they must accomplish during the day such as medications, the technology, and the process that work as a nurse consists of activities on a check list which must be accomplished (Carter, et al., 2008; Woodgate \& Kristjanson, 1996a). Observations accounts have reported technology as a ubiquitous part of the ER (Wolf et al, 2003). Nursing entails a great number of specific technical skills which fills the nurses' day, routine tasks done dozens of times (Chambliss, 1996; Fisher \& Peterson, 1993). The repeatability of procedures and tasks distances the nurse and fosters an attitude of detachment and unconcern for the patient (Chambliss, 1996).

Detachment and procedural focus has been reported in the attitudes of surgeons, surgical and emergency nurses. A qualitative study exploring surgeons' behaviors reported that physicians detached themselves emotionally to be able to focus on the technical aspects of the procedure (Fisher \& Peterson, 1993). In a qualitative study, nurses in the accident and emergency department discussed their perceptions of their work and how it influenced patient communication and care (Byrne \& Heyman, 1997). Despite the relative rarity, caring for major trauma patients was identified as an aspect of emergency work valued by almost all emergency nurses. An important feature of major trauma patients was that they allowed nurses to exercise certain practical, often technical skills that provided nurses with the opportunity to feel both technically expert and 
rewardingly useful. This result was similar in a sociological study where nurses in areas of high acuity and skill needed a crisis fix to work with the desperately ill or injured and thrived on the excitement of the crash room and code (Chambliss, 1996). The researchers concluded that the emphasis on major trauma may have contributed toward the limited attention provided to non-urgent patients (Byrne \& Heyman, 1997).

Surgical nurses reported detachment served a functional purpose, the utility of desensitizing themselves emotionally and maintaining a professional distance allowed surgical nurses to perform more efficiently (Fisher \& Peterson, 1993). The deindividualization of patients has been reported in other studies (Byrne et al., 2001; Chambliss, 1996) were patients were viewed as procedures rather than as people. Bryne, Morton, and Salmon (2001) reported that nurses denoted children as the body part that had been operated upon, children's attempts to negotiate with the nurse were negated, and individuality was negated by routinization of care, particularly in the timing of common procedures. Chambliss (1996) noted that the patient becomes an object of looking and talking, and the extensive division of labor exposes patients to the process production of the unit routines in which each member of the staff has some particular job to do on them. Interview results of patients' experiences of being a non-urgent emergency department patient reported that they had feelings of detachment and experienced themselves being considered as an object by the nurses and not as an individual (Nyström, Nydén, \& Petersson, 2003).

\section{Nurses' Intuition}

Previous research indicates pain rating scales for children are not widely used (Jacob \& Puntillo, 1999; Margolius, Hudson, \& Michel, 1995; Schmidt, Holiday, Kleiber, 
Peterson, \& Phearman, 1994), and that pain scores appeared to represent nurses' intuition of a child's pain with no clear parameters of how pain intensity was assessed (Byrne et al., 2001; Twycross, 2007a).

Chambliss (1996) noted that in the hospital the nurses and doctors are in charge, so their view of what is right prevails. Research studies support that nurses' and physicians' rely on their value judgment of patients pain to guide treatment (Byrne et al., 2001; Howell, Butler, Vincent, Watt-Watson, \& Stearns, 2000; Matthews \& Malcolm, 2007; McCaffery, Ferrell, \& Pasero, 2000; Twycross, 2007a).

Byrne et al. (2001) used structured and unstructured observation to assess how nurses managed post-operative pain in children and whether practices adhered to best practice guidelines. They reported that nurses viewed and managed children according to a schedule of objective behavioral milestones that denied the reality of urgency of children's pain and distress. McCaffery, Ferrell, \& Pasero (2000) utilized surveys to examine nurses' decisions about assessment and treatment of pain in patient situations. Survey results revealed that nurses' personal opinions about the patients' pain, rather than their recorded assessment influenced choice of opioid dosage. Howell, Butler, Vincent, Watt-Watson, and Stearns (2000) used survey and chart audit to examine the effect of education intervention in changing the knowledge, attitudes, and practice behaviors of registered nurses' in the assessment and management of cancer pain. They found while nurses acknowledged patient's self-report as the most accurate measure physician and nurse pain ratings were more valid.

Several studies have reported that healthcare providers' personal opinion of a patients' pain intensity is an inaccurate indicator, and frequently underestimates the 
patients' pain experience (Guru \& Dubinsky, 2000; Kotzer, 2000; Maciocia, et al., 2003;

Puntillo, Neighbor, O’Neil, \& Nixon, 2003; Romsing, Moller-Sonnergaard Hertel, \& Rasmussen, 1996; Singer, Richman, Kowalska, \& Thode, 1999). Singer, Richman, Kowalska, and Thode (1999) used observation and survey to compare patient and practitioner assessments of pain associated with commonly performed emergency department procedures. They reported practitioners' predictions correlated poorly with the patient's own assessments regarding the amount of pain experienced. Maciocia et al (2003) used observation and survey to compare patient, guardian, and professional assessment of acute pain in children presenting to an emergency department. The results revealed professionals scored pain lower in comparison with children and guardians. The researchers' concluded professionals' greater experience of children in pain enabled them to prescribe the appropriate level of analgesia required for each child.

In an observational study of emergency physicians' assessment and treatment of pain, physicians were documented to make no inquiry about the quantity of pain, yet recorded pain severity on the patients' chart, and treatment was prescribed for the obviously painful conditions (Chisholm, Weaver, Whenmouth, Giles, \& Brizendine, 2008). Guru and Dubinsky (2000) examined how emergency department caregivers interpret and treat acute pain. Pain ratings of patient, nurse, and physician were obtained independently. They reported that both physicians and nurses perceived the patients' pain level to be lower than the patients' own rating of their pain. Nurses' ratings were found to be lower than physician ratings of the same patient. 


\section{Non-Pharmacological Methods}

Some researchers have criticized the use of non-pharmacological methods. In a guest editorial, pain researcher McCaffery (2002) wrote due to the continued lack of clinicians' assessment and reassessment of patients' pain, and the inefficient use of opioids, spending time and money to incorporate non-drug methods for pain relief into treatment is hardly justifiable.

Several observational studies of post-operative nurses' pain management practices reported non-drug methods were not routinely used pain-relieving methods (Twycross, 2002, 2007a, 2008; Woodgate \& Kristjanson, 1996a, 1996b) and when nurses had the opportunity to interact with children nurses' were observed on many occasions to sit around the nurses' station (Twycross, 2002, 2007a). This finding is inconsistent with other studies which reported nurses' identify lack of time to provide psychological support as a common barrier (Pack-Mabien et al., 2001). Meunier-Sham and Ryan (2003) developed developmentally appropriate strategies to decrease the pain associated with routine and emergency pediatric procedures. Results revealed that staff believed non-pharmacological methods were helpful but inadequate. Other researchers' who examined pain management practices through chart review were not able to ascertain whether non-pharmacological methods were implemented due to lack of documentation of such practices (Cohen, et al., 2003; Todd et al., 2007).

Twycross (2007b) proposed that nurses hold a one-sided view of pain management seeing the nurse's role in pain management as synonymous with the administration of analgesic drugs. Using observation and interviews, Woodgate and Kristjanson (1996a) explored how parents and nurses responded to hospitalized young children experiencing 
pain from surgical interventions. They found that analgesic administration to be the most frequent practice carried out by nursing staff.

Nurses' knowledge deficit in the use of non-pharmacological pain relieving techniques has been found to potentially impede implementation of such practices. Results from two surveys reported that nurses' did not know that non-drug interventions could be beneficial in the treatment of severe pain, as well as mild to moderate pain, and that heat and cold could be effective when applied to a non-painful area (Comley \& Banks, 2000; Matthews \& Malcolm, 2007).

Research findings support that despite nurses stating that they use nonpharmacological methods such as distraction, positioning and preparatory information to relieve child's pain, non-pharmacological methods were not found to be regularly implemented (Hamers, Abu-Saad, Halfens, \& Schumacher, 1994; Twycross, 2007a; Woodgate and Kristjanson, 1996a, 1996b). An exploratory study which examined the factors influencing nurses' pain assessment and decision-making revealed distraction as the most used non-pharmacological intervention; however, the use of other nonpharmacological strategies were not implemented (Hamers et al., 1994).

\section{Attitudes towards Pediatric Pain Management}

A belief system can be defined as the basic set of values and assumptions that a person holds to be true regarding pain, suffering, treatment, and analgesia which may or may not be based on fact (Greipp, 1992). Researchers have proposed that attitudes and personal beliefs about pain and pain relief influence pain management practices (Broome \& Huth, 2003). In a joint position statement, the American Academy of Pediatrics and the American Pain Society (2001) identified that barriers to the treatment of pain in children 
include the myths that addressing pain in children takes too much time and effort, children do not feel pain the same way adults do, and there are no negative consequences to untreated pain.

An extensive body of research is available on practitioner pain management attitudes, knowledge, and beliefs (Comley \& Banks, 2000; Jacob \& Puntillo, 1999; PackMabien et al., 2001; Tse \& Chan, 2004; Visina, Chen, Gerthoffer, Biggs, \& Ting, 2003; Zanolin, et al., 2007). Clinicians' with incorrect assumptions about pain in children and in pain-denigrating attitudes will interpret pain behaviors with that framework in mind (Schechter, 1989). Survey results of community hospital physicians' and nurses' attitudes about pain management employed by Visina, Chen, Gethoffer, Biggs, and Ting (2003) reported that clinicians' believed patients should tolerate low amounts of pain without medication, staff believed that they were adequately managing their patients, and satisfied with pain management outcomes. This is evident from studies in which nurses' reported pain was unavoidable and over quickly (Meunier-Sham \& Ryan, 2003), and the use of analgesia was not justified when it was considered to add another poke (Brown, 2002; MacLean et al., 2007). Read (1994) utilized a survey to examine the perceptions of pain and pain management by nurses and physicians who cared for pediatric emergency room patients. The results reported incongruence between physicians and nurses responses on pain management items on the questionnaire then with the actual practice.

Another factor which has been reported to affect the healthcare providers' interpretation of pain is the medical diagnosis. Research findings support that there is a perceived hierarchy of suffering identified based on diagnosis (Chisholm et al., 2008; Hamers et al., 1994; Woodgate \& Kristjanson, 1996a). Therefore, patients who have 
undergone more extensive procedures or have perceived painful injuries are more likely to receive higher pain ratings by healthcare providers, and more pain relieving treatments. Hamers, Abu-Saad, Halfens, and Schumacher (1994) reported that nurses' attached a great deal of importance to the medical diagnosis, and the presence of a medical diagnosis for which pain is expected seemed to allow and justify the patient being in pain.

Several studies have documented that clinicians' tend to rate patients pain based on overt pain behaviors (Byrne et al., 2001; Comely \& Banks, 2000; Hamers et al., 1994; Matthews \& Malcolm, 2007; McCaffery et al., 2000; Tse \& Chan, 2004). Brown (2002) reported registered nurses' felt professional judgment and observational assessment were sufficient determinants to define patient need for pain management. Survey results of nurses' attitudes toward pain assessment tools conducted by Young, Horton, and Davidhizar (2006) reported nurses’ believed pain assessment tools as subjective, inaccurate, and preferred assessing mannerisms as the better alternative. Survey results of nurses' knowledge and attitudes conducted by Simpson, Kautzman, and Dodd (2002), reported nurses' and other staff persisted in their beliefs that vital signs were a more reliable indicator of pain than the patient's own self-report even after 2 years of staff education. The use of pain assessment tools has been reported by clinicians' to be unimportant, not beneficial, and useless (Drendel et al., 2006; Young, Horton, \& Davidhizar, 2006). Pain assessment tools have been criticized for being time-consuming and impractical for providers in a busy emergency department (Drendel et al., 2006). Clinicians' in the emergency department setting believed that prolonged time taken for pain management techniques disrupted patient flow (MacLean et al., 2007). 


\section{Contextual Influences}

Pain management practices are influenced by the context of environmental demands such as high patient loads, legitimate needs of other patients, economic constraints and the workplace priority placed on pain management (Walker, Tan, \& George, 1995). Reasons cited in the empirical literature for not using pain-relieving techniques have included no options available, need for another staff member to help, time consumption, heavy workloads, and patient turnover rate (Broome \& Huth, 2003; Meunier-Sham \& Ryan, 2003; Pölkki, Laukkala, Vehviläinen-Julkunen, \& Pietilä, 2003; Schafheutle et al., 2001).

Workload and lack of time have been identified as barriers to optimal pain management practices (Dalton et al., 2001; Kaasalainen et al., 2007; Pack-Mabien et al., 2001; Schafheutle et al., 2001). Kaasalainen et al. (2007) explored the decision-making process of physicians and nurses in pain management for older adults with cognitive impairment. The results revealed that nurses were constantly working under immense pressure to meet the demands of their workload and had difficulty including time to adequately assess patients' pain or provide psychosocial support. Puls-McColl, Holden, and Buschmann (2001) reported the greatest barrier of floor nurses to be the responsibility of caring for other acutely ill patients, and the lack of time to adequately assess and control pain.

The unpredictable pace of the emergency department has been reported to be a contributing factor to what structures the focus of patient care (Byrne \& Heyman, 1997; Smith \& Feied, 1999; Wolf et al., 2003). Unlike any other unit in a hospital setting, the emergency department is a self-contained complex unit open 24 hours of the day every 
day with patients continuously waiting to be seen and treated (Smith \& Feied, 1999). The main purpose of the emergency department has been identified as saving lives and issues related to emotional or mental health have been categorized as trivial (Hemmelgarn et al., 2001). In one study, accident and emergency department nurses viewed their priority as the provision of urgent physical care which left insufficient time to deal with each patient individually (Byrne \& Heyman, 1997). This was supported by statements from interviews with non-urgent patients in the emergency department that nursing personnel had little time to care for lowly prioritized non-urgent patients (Nyström, Nydén et al., 2003).

Emergency department nurses and physicians have been identified to experience various time pressures to act, driven by how patient conditions unfold or present themselves, variables which cannot be predicted in advance (Smith \& Feied, 1999). The uncertainty of diagnosis has been reported to influence pain management care. One study of pediatric emergency physicians reported that providing analgesia for children with acute abdominal pain was strongly influenced by pediatric surgeons' strong opposition to provide analgesia before surgical intervention (Kim, Galustyan, Sato, Bergholte, \& Hennes, 2003). The uncertainty of events such as ambulance calls, major trauma, and resuscitation codes have been reported to represent situations where accuracy and time could not be compromised (Wolf et al., 2003).

Patient volume, workload, and competing priorities have been reported to be contributing factors (Byrne \& Heyman, 1997; Ellis et al., 2007; Olive, 2003; Probst et al., 2005; Puls-McColl et al., 2001; Smith \& Feied, 1999) to the under management of pain. Byrne and Heyman (1997) reported that nurses felt it was more important to move patients through the emergency department. Wolf, Evans, Hewitson, and Perez (2003) 
reported that nurses pressured physicians to clear the emergency department to keep the ER open.

The lack of priority given to pain management has been reported to affect care. Woodgate and Kristjanson (1996a) reported that many other symptomatic states experienced by children received more attention than pain symptoms. Twycross (1999) reported that nurses perceived pain management to be a less critical aspect of the nursing role than their clinical role. Hemmelgarn, Glisson and Dukes (2001) examined the role played by organizational culture in the emphasis that emergency room staff place on the emotional support component of family-center care by studying four different emergency room cultures. They reported that staff descriptions of the emphasis placed on emotional support to the families of children who received emergency care was found to be a function of the ER in which staff worked and not of clinicians' experience, profession, or sex. Meehan, McRae, Rourke, Eisenringer, and Imperial (1995) reported if nurses’ worked in an environment that valued the importance of pain management, were encouraged by supervisors and peers, then nurses' were more likely to improve pain management practices.

\section{Conclusion/ Major Gaps}

In summary, the practice of emergency medicine is quite different from other areas of practice and some of the reasons for the under management of pain may also be distinctive (Johnson, 2005). Because of the limited empirical research on obstacles to effective pain management in the emergency department (Byrne \& Heyman, 1997; Olive, 2003; Tanabe \& Buschmann, 2000), rationales given for the phenomenon in emergency medicine emerge from the intuition and experience of emergency providers (Smith \& 
Feied,1999) or are extrapolated from the limited studies conducted in the emergency department setting that exist (Johnson, 2005).

Few studies were located which examined pediatric procedural pain management practices in the emergency department setting. Of those studies located, examination of pediatric procedural pain management in the emergency department has been limited to emergency departments located in pediatric hospitals (Bhargava \& Young, 2007; MacLean et al., 2007; Meunier-Sham \& Ryan, 2003). The majority of studies conducted in the emergency department setting (Alexander \& Manno, 2003; Cimpello et al., 2004; Fry, Holdgate, Baird, Silk, \& Ahern, 1999; Johnston, Bournaki, Gagnon, Pepler, \& Bourgault, 2005; Kim et al., 2003; Todd, et al., 2007; Wong, Chan, Rainer, \& Ying, 2007) examined practice patterns of clinicians' treatment of acute pain as a primary complaint such as abdominal pain, or fracture.

One article was located which examined how nurses and physicians perceived and managed procedural pain in children in the emergency department setting. Read (1994) used survey which examined attitudes and perceptions of nurses and physicians regarding premedication practices with pediatric patients undergoing procedures. The researcher reported responses on the pain management items of the questionnaire were incongruent with the actual practice; while over $95 \%$ indicated they would medicate the children described in the vignettes presented, in actual practice clinicians treated 5-6 children per day not medicated for acute pain. Based on those findings, the concern is raised that information obtained by surveys may reflect a social desirability response. None of the previous research has observed how clinicians manage procedural pain of pre-operational children in the non-pediatric emergency setting. 
Much of the empirical research literature that has influenced pain management practices has been formulated under the assumption that an individual's attitudes and beliefs influence pain treatment practices. While an individual's attitudes and beliefs may account for some pain management practices, other researchers have argued for the concept of culture (Foster, 1991) and suggest that groups make meaningful adaptations to their environments (Schein, 1992). Based on this argument pain management practices may be driven by situational contingencies that arise from the immediate external environment (Schein, 1992). Many of the studies reviewed failed to take into consideration the cultural and psychosocial affects of the unit culture. Given this information, further research is required. All of the above mentioned areas relate to a larger paradigm where ritual, values, and behaviors bind together into a coherent whole called culture (Schein, 1992).

\section{Theoretical Foundations of Ethnography}

Ethnography is located within the approach of naturalism where the intention is to collect data from real life situations which are undistorted by the researcher, in a way which conveys the subjective reality of the interior world of the participants (Pole \& Morrison, 2003). Matza (1969) characterized naturalism as the philosophical view that remains true to the nature of the phenomenon under study. Naturalism proposes that the social world should be studied in its natural state undisturbed by the researcher, and in order to understand people's behavior an approach which gives access to the meanings that guide behavior are required (Hammersley \& Atkinson, 1983).

Ethnography is not only a method of data analysis, but also a way of conceptualizing and looking at a particular group or culture (Fetterman, 1998; Spradley \& McCurdy, 
1972; Spradley, 1980; Wolcott, 2008). As a research process, ethnography is the systematic collection, description, and analysis of data to discover the underlying knowledge system a group of people have learned and apply to organize their behavioral patterns (Spradley \& McCurdy, 1972; Spradley, 1980). Ethnography consists of the process of observing human behavior in a holistic cultural context where the domain of observation is culture as manifested by the native's verbal and nonverbal behavior (Shimahara, 1984). The methodological orientation of ethnography consists of assumptions about group culture which include the observation of events relevant to the framework of an immediate research setting and relationships to a broader sociocultural milieu where events cannot be isolated from the context in which it originates (Pole \& Morrison, 2003; Shimahara, 1984).

Shimahara (1984) identifies three paradigmatic premises which underlie the qualitative orientation of ethnography: (1) ethnographic research emphasizes the integration of particular events into a coherent and qualitatively meaningful pattern where the relationship of events is established, (2) cultural events are understood and categorized in terms of the cultural actor's definition of human events where attention is focused on how they define their reality, and (3) ethnographer's focus on ongoing settings in sociocultural contexts where events occur as human interaction takes place, these settings are natural in the sense that they are not contrived or modified by the observer.

Aamodt (1982) provides a description of four assumptions of ethnographic research. The first assumption reads cultural rules inform human social behavior, that is, human behavior is derived in part from a cultural system. The cultural systems all human beings 
are socialized into provide a framework for human activity in multiple cultural scenes of day-by-day activities. Without a cultural system to inform us chaos would ensue. Cultural behavior is generated by learning and following the instructions of cultural rules to behave in a particular way. The rules of a particular cultural system tell the participant culturally appropriate ways to behave.

The second assumption reads that cultural data derives from abstractions of behavior about what people do and what they say they do (Aamodt, 1982). Culture as a behavioral system and culture as a cognitive system represent two major theoretical approaches of cultural anthropologists. Behavioral conceptualizations focus on the observable patterns of behavior among members of a social group. Cognitive conceptualization of culture focuses on the ideational system of a society. Most ethnographers use both approaches in data collection and analysis to learn about what people do and what people say they do. Abstractions of this information are made by ethnographers who organize their view of cultural system in a form usable by members of the clinical nursing research community (Aamodt, 1982).

The third assumption states the ethnographers are active participants in both data collection and data analysis (Aamodt, 1982). Ethnographers incorporate the influence of their participation in what they are observing with the conceptual base of the investigation.

The fourth assumption states the analytical processes in doing ethnography focus on generating categories and discovering relationships between theses categories (Aamodt, 1982). The intent of ethnography is to provide a systematic, baseline detail grounded in the culture concept for the continuing generation of hypotheses and theory. For nursing, 
this means an opportunity to identify and describe concepts and constructs that are culturally relevant in caretaking scenes of self and others (Aamodt, 1982).

Ethnography is an invaluable method to explicate nursing phenomena (Leininger, 1995). Ethnography attempts to learn what knowledge people use to interpret experience and mold their behavior within the context of their culturally constituted environments (Aamodt, 1991). The goal for nurse ethnographers is to continually seek out the meanings in the native's view in cultural and multicultural settings and search for ways of linking these understandings with concepts, processes and models for intervention in nursing care delivery systems (Aamodt, 1982).

Ethnography is selected because the method is a systematic attempt to learn cultural meanings and motives which lie at the base of social actions. Ethnography is the study of culture and provides detailed, descriptive data that can be used as background information for understanding human behavior by practicing nurses and clinicians (Aamodt, 1982). The knowledge and theory base of nursing needs to be expanded by research which specifically aims to investigate the cultural context associated with pediatric procedural pain management in the non-pediatric emergency department unit. Ethnography is a research approach eminently suited to this purpose.

In ethnography, researchers are the instruments for data collection (Roper \& Shapira, 2000). An ethnographer is a human instrument who must discriminate among different types of data and analyze the relative worth of one path over another while in the field (Fetterman, 1998). The human instrument is a most sensitive and perceptive data gathering tool who relies on all senses, thoughts, and feelings (Fetterman, 1998). Immersion of the researcher through direct observation, participation, interviews and 
examination of documents provides the researcher with the ability to write a thick description of what has been heard and seen within the framework of the social group's view of reality (Fetterman, 1998; Morse, 1991; Roper \& Shapira, 2000; Spradley \& McCurdy, 1972; Spradley, 1980).

Culture exits at the explicit and tacit levels (Spradley \& McCurdy, 1972; Spradley, 1980). Explicit cultural knowledge is more overt and easily communicated. Tacit knowledge is outside of a person's awareness. As a participant observer, the ethnographer is introduced to the linguistic expressions that convey the meaning of objects and events (Aamodt, 1991). Ethnography is an inclusive research endeavor which involves the researcher as a resident of the group over an extended period of time where participant observation is requisite to obtain first-hand knowledge of the native's organizations and functions (Fetterman, 1998; Shimahara, 1984; Spradley \& McCurdy, 1972; Spradley, 1980). The perspectives of the observer and the observed are intertwined in the generation of questions and hypotheses (Shimahara, 1984). Ethnographers' commitment is to immerse themselves in the host culture, remain detached as intense observers inquiring into cultural phenomena, maintain neutrality and have a multidimensional vision of both observing and interacting with research participants (Shimahara, 1984). This suggests that the researcher has to focus on the entire group and wide range of informants representing the group (Shimahara, 1984).

Triangulation of the data uncovers the tacit knowledge, the hidden principles of the way of life, and the taken for granted ideas and practices (Lofland, Snow, Anderson, \& Lofland, 2006; Spradley, 1980) that influence the way pediatric procedural pain management is delivered and constructed in the non-pediatric emergency setting. The 
centrality of culture as knowledge and value orientations has been argued to be of fundamental importance in motivating behavior because the emphasis is placed more on the meaning behind action, events, beliefs, and values (Kluckhohn \& Strodbeck, 1973; Spradley \& McCurdy, 1972; Spradley, 1980). In this study, ethnography goes beyond patterns of observed behavior and strives to inquire about the meaning or underlying value of the behavior. Through the examination of both what people say and what people do may make it possible to understand the everyday routines and conditions (Fetterman, 1998; Spradley \& McCurdy, 1972; Spradley, 1980) under which healthcare providers conduct themselves in the non-pediatric emergency department, and the constraints and pressures to which such conduct is subject (Emerson, Fretz, \& Shaw, 1995).

This ethnography will provide a comprehensive and contextualized description of the non-pediatric emergency department, and discover the meaning of observed patterns of behavior in pediatric procedural pain management. The description and analysis of the unit culture of the non-pediatric emergency department and how the non-pediatric emergency department manages pediatric procedural pain may provide a useful baseline against which changes in unit culture can be evaluated and hypothesis generated. This information may be valuable to healthcare professionals, nurses, and physicians who want to change practice patterns, and better pediatric procedural pain management in the non-pediatric emergency department.

The limitation of conducting ethnography in a single non-pediatric emergency department may limit the generalizability of the data. Brewer (2000) argues because ethnography involves a restricted setting, ethnographic research is best at making theoretical inferences than at applying data to a wider population. Wolcott (2008) 
encourages ethnographers to share generalizations with the important point to state the basis on which generalizations have been formed and the extent to which they include impressions, emotions, and whatever other personal resources have been drawn upon.

Another limitation of ethnography may include the presence of the researcher in the natural setting. The ethnographer's presence may affect the behavioral conduct of research subjects by making them feel self-conscious and perform better than normal, also referred to as the Hawthorne effect (Polit \& Beck, 2008). Twycross (2007a, 2007b) reported that with prolonged engagement nurses' on the pediatric floor habituated to the presence of the observer and that pain management practices obtained during the study were probably reflective of true practice.

The formal literature review will be conducted during the data analysis and interpretation phase of the study (Munhall, 2007). The literature review will be grounded in the collected data so that findings can be compared with previous literature, and incorporate conceptual framework(s) that facilitate understanding of pediatric procedural pain management within the non-pediatric emergency department. 


\section{Chapter III}

\section{Method}

The purpose of this research was to describe the unit culture of the non-pediatric emergency department, explore the ways healthcare providers evaluate and treat pediatric procedural pain, and explore attitudes of healthcare providers towards the treatment of procedural pain in the pre-operational child. This includes a conceptual description of how clinicians interact in terms of what they say, what they do, and the shared inferred expectations they hold for pediatric procedural pain management in the pre-operational child (Wolcott, 2008). To achieve this purpose, ethnography was adopted as the research design.

Ethnography is a research process fundamentally rooted to the disciplines of anthropology and sociology. The shared commonalities between these two disciplines are focused on the task of cultural description (Brewer, 2000). Leininger (1970) defined the use of ethnography in nursing as the systematic process of observation, detail, documentation and analysis of particular patterns within a culture or group in order to grasp the patterns of the people in their familiar environment.

Ethnography as a method is a means for gaining access to the beliefs and practices of a culture by establishing the trust necessary for informants or actors to reveal material which they might be hesitant to share in other research situations, and for understanding the emic point of view (Robertson \& Boyle, 1984). The emic perspective represents the way members or insiders' of a particular group see, interpret, and understand the world around them (Brewer, 2000; Pole \& Morrison, 2003; Roper \& Shapria, 2000). This is in contrast to the etic perspective that represents the outsiders' interpretation of viewed 
experiences from the observed culture (Brewer, 2000; Pole \& Morrison, 2003; Roper \& Shapria, 2000). For this ethnography, both the emic and etic perspectives were utilized.

\section{Research Questions}

In ethnography, questions formulated prior to actual fieldwork are subject to change as the study progresses (Morse, 1991; Roper \& Shapira, 2000). Although the researcher was guided by specific questions, there were no minimal preconceived notions about the outcomes of the research (Roper \& Shapira, 2000). The main research question was formulated as: What social and cultural factors influence healthcare providers' evaluation and treatment of pediatric procedural pain in a non-pediatric emergency department?

\section{Guiding Questions}

This study was guided by the following questions:

1. How are healthcare providers pediatric procedural pain management behaviors organized within the non-pediatric emergency department?

2. How is pediatric procedural pain management perceived by healthcare providers in the non-pediatric emergency department?

3. What are the unwritten rules for managing pediatric procedural pain in the nonpediatric emergency setting?

4. How are these unwritten rules for managing pediatric procedural pain in the nonpediatric emergency setting perceived by members of the emergency staff?

5. What are the pediatric procedural pain management practices of emergency staff in a non-pediatric emergency department? 
6. What are the beliefs regarding pediatric procedural pain management of emergency staff in the non-pediatric emergency department?

7. What tools are in place to assist the emergency staff in managing pediatric procedural pain in the non-pediatric emergency setting?

\section{Locating a Social Situation}

The first step in doing ethnography is to locate a social situation. Every social situation can be identified by three primary elements a place, actors, and activities (Spradley, 1980). The social situation in which the researcher was concerned involved a non-pediatric specialized hospital, emergency clinicians' and their pediatric procedural pain management practices. The researcher selected Fellowship Hospital as the site for the study based on the criteria that it had no formal separate pediatric designated area in the ED, no formal designated pediatric staff, and the percentage of pediatric visits through the ED were the highest compared to other general ED's that met the same criteria. Access to the hospital organization was made possible through contact with the chairperson of the nurse research council (NRC). The Chairperson of the NRC was the main gateway person into the organization.

\section{Participant Selection}

Purposive sampling was used for this qualitative study. Initially, the researcher located key informants registered nurses, physicians, mid-level practitioners, medical technician and nurses' aide with general knowledge of the topic and who had undergone the experience. Then as the study progressed and the description, categories, and meanings were expanded with more specific information, participants with that particular knowledge were sought out (Morse, 1991). The researcher's goal was to observe and 
interview participants about pediatric procedural pain management within the context of the non-pediatric focused ED.

A hallmark of ethnographic field work is locating informants. During the start of observations in the ED, the researcher was approached by different healthcare providers interested in the study. The researcher gained their cooperation and established a working relationship with them (Fetterman, 1998) to learn about the ED environment and their practices. Spradley and McCurdy (1972) describes characteristics to look for in an informant as one who knows the culture well, is willing to talk, and who communicates about their culture in a non-analytic manner. Good informants are not just those who have experienced the phenomena under study, but those who are willing and able to discuss their experience (Mackenzie, 1994). The researcher proceeded to do purposeful sampling and continuously sought out participants whose experiences were indicative of theoretically relevant concepts.

\section{Inclusion Criteria}

The sample included clinicians and ancillary personnel registered nurses, advanced practice nurses, physician assistants, physicians, medical technicians, and nursing assistants in the ED who provided for or managed pediatric pain before, during, and after a procedure.

Inclusion of children in this research involved observations of healthcare provider and child interaction before, during, and after procedural pain management and review of healthcare provider documentation related to child's procedural pain management. 


\section{Protection of Human Subjects}

Approval from the institutional review board (IRB) of Rutgers University Human Subject Research Committee and Fellowship Hospital was sought out and obtained prior to the initiation of the study (Appendix A).

Potential participants included both healthcare providers in the ED and children and their guardians. The possibilities of harmful effects from ethnographic studies are relatively minimal and any discomfort the participants experienced were no greater than ordinary encounters in daily life (Roper \& Shapira, 2000). In ethnographies, the primary violations consist of privacy or confidentiality. In this study, there was a risk of breach of confidentiality for the patients and their families as the researcher would have access to medical information about their children. Prior to the recruitment of participants, parents and children were approached by the researcher and told the purpose of the study was to learn how ED staff assessed and managed procedural pain in children. Written inform consent was developed for the families and patient to be aware of the study objectives, risk and benefits, as well as what would be involved, and that participation was voluntary and could be terminated at any time. Consent was obtained from the parent or guardian of the pediatric patient that allowed the researcher to review the patients chart related to procedural pain assessment and pain management, and when possible observed interactions of healthcare providers with the pediatric patient before, during, and after procedures. Once a patient was identified and consent obtained, the researcher focused observations on that individual case until the patient was discharged from the ED or admitted off the ED unit or until the researcher left for that particular day. No personal or identifying information was gathered from the patient. No retrospective chart review was 
conducted. Due to the emergent nature of the emergency department environment, only when a patient's chart was available during the same observation session did the researcher review data on the chart related to pain assessment and management.

For healthcare provider participants, written informed consent was also obtained. Pseudonyms were provided to the institution and all participants so that they could not be identified when results were reported. Included in the consent for providers was a separate consent to audio-taping of the interview data. Participants were assured that all interviews were kept in the strictest confidence, and that participation was voluntary and could be terminated at any time. If a healthcare provider declined for the interview to be audio-taped, but agreed to sit for the interview paper and pen notes were taken. No participants in this study refused to be audio-taped. Demographic details were separated from other data to ensure that participants could not be identified. Information was stored on a personal computer accessible only to the researcher which was encrypted and password protected. All files and audiotapes were stored in a locked key coded file cabinet located in the researcher's home. Only the researcher had direct access and her committee members would have access to the files by request as necessary.

\section{Data Collection and Analysis}

In ethnography, data analysis occurs simultaneously and continuously with data collection (Fetterman, 1998; Pole \& Morrison, 2003). Ethnographic analysis is inductive and iterative it involves building on ideas throughout the study, and searching through collected field notes to discover the cultural patterns of the social situation. In this study, the researcher undertook an intensive analysis of the data collected each time before reentry into the field (Spradley, 1980). The purpose was to organize the data and then 
make sense of what had been learned during the research experience. In this way, theories and patterns were explored, developed, or hypothesized; potential sources of bias and inconsistencies in the data were uncovered and dealt with as part of the ongoing research process (Robertson \& Boyle, 1984).

Sources of data for this study included field notes from participant observation, demographics of healthcare providers, interviews with healthcare providers who participated in painful pediatric procedures and documents that included institutional policies on pain management, ED unit specific policies on pain management, ED unit policies for triage, treatment algorithms, procedures for pain management, organizational flow charts, staffing assignments and documentation on procedures or pain management in the patient record.

Data were collected mainly through observations of healthcare providers' interactions before, during, and after management of pediatric procedural pain in the emergency department setting. Observations were made at least three times per week for periods of time from 4 to 7 hours. Field notes were written within 24 hours of the observation. In order to ensure a full accurate description of the phenomenon under study, data collection was continued until repetition of data occurred without any new categories or themes. In this study, the collection of data took place over a 5 month period in order to achieve repetition of data and achieve theoretical saturation.

\section{Field Notes}

In this study, condensed notes were used to quickly record observations, key phrases, and single words or identified major events that occurred during the observation periods and served as useful reminders to later create an expanded account. The expanded field 
note was written as soon as possible after each session. The researcher set aside time after each scheduled observation to fill in details and recall things that were not recorded on the spot. This documentation is referred to as substantive field notes of the day-to-day activities of participant observation (Saldaña, 2009). The researcher recorded vivid and detailed information that included actions and interactions of providers with children and other members of the unit (Spradley, 1980). On each return visit the researcher observed activities that appeared similar, if not identical, to what occurred earlier. The continual observation of participants' routines and practices, and repeated description in field notes allowed the researcher to discover the complexity of how healthcare providers assessed and managed pediatric pain in the context of the non-pediatric ED environment (Spradley, 1980).

\section{Field Journal}

A reflexive field journal was maintained by the researcher that contained the personal side of fieldwork. In this study, each journal entry was dated and documented the impressions, feelings, emotions, the development of relationships during observation, the emotional costs and problems in the field and other exigencies that affected research (Fetterman, 1998; Spradley, 1980).

\section{Analytic Field Note}

The analytic field note is where the researcher records generalizations, analyses of cultural meanings, interpretations and insights into the culture studied (Spradley, 1980). Researcher notes represented brainstorming on paper about the culture under consideration and recorded the initial tentative interpretations (Spradley, 1980). 


\section{Demographics}

Demographic data was collected from the healthcare provider participants.

Demographic information collected included: job title, ethnic/ racial background, gender, age, work experience, previous pediatric experience, and whether the healthcare provider was a parent (Appendix B).

\section{Interviews}

Interviews were used to validate observations made during ethnographic participant observation and provided direction for future observations. They were also used to gather data related to issues that the researcher may not have observed, or could not observe such as the participants' thoughts, or that could not reliably be ascertained by observation, the participants' feelings. In this study, both informal and semi-structured interviews were utilized.

Informal interview involved asking clinicians' questions about an event or interaction immediately after it occurred in order to check the participants' perceptions against the researchers'. Informal interviews were useful in discovering what clinicians' thought and how one clinician's perceptions compared with another's. Such comparisons helped identify shared values in the community-values that informed behavior. Informal interview was also useful in establishing and maintaining a healthy rapport (Fetterman, 1998).

The utilization of semi-structured interviews toward the middle and end stages of the study have been found useful for the collection of data about a specific question or hypothesis (Fetterman, 1998), as was the case in this study, which then allowed for the development of other questions. After the researcher had spent some time in the field and 
had a general understanding of the emergency department and patterns of interaction among clinicians' specific questions were developed and a semi-structured interview format was utilized with key informants. An interview guide was used to help clinicians' openly discuss issues specific to pediatric procedural pain in the non-pediatric emergency department. The preliminary interview guide is included (Appendix C).

\section{Existing Documents}

The analysis of supplementary or existing documents, as additional sources of information, are important to capture the rich meaning and essential patterns of participant behavior and beliefs (Roper \& Shapira, 2000). In this study, documents included but were not limited to policies of pain management, procedures for pain management, documentation of procedural pain treatment and disposition in nurses' and physicians' notes, staffing assignments, organizational charts, and flow charts (Brewer, 2000; Roper \& Shapira, 2000).

\section{Case Study}

All ethnographic research involves case study. Ethnographic case study is distinguished by exploration of the case or cases as they present themselves naturally in the field and by the researcher's direct involvement and participation in them (Brewer, 2000). The researcher observed clinicians interaction before, during, and after procedural pain management of preoperational children in the non-pediatric emergency department. For this study, the researcher was positioned in the exam room during procedures in a way that interaction and treatment were seen and heard without interruption of the normal ED process. 


\begin{abstract}
Analysis
The process of data analysis followed the procedure developed by Leininger and McFarland (2006) which provides for a four sequenced phase of analysis. Phase one is the collection, description, and documentation of raw data and follows with data coding, processing, and analysis of all data.

In phase one the analysis of the detailed raw data is performed before moving to the second phase. Data analysis began from the first observation when the field notes were expanded, typed, and read before the next visit to the field (Brewer, 2000). Semi-formal interviews were tape recorded and transcribed by the researcher into verbatim scripts as soon as possible following the interviews. The researcher read the scripts several times while listening to the tape to make sure they corresponded with what the participants said. To organize the data, The Ethnograph version 6.0 database software program (Seidel, 1998) was utilized. This software program is suited to ethnographic research because it allows the researcher to enter long field notes without difficulty, code, retrieve, and analyze data (Fetterman, 1998). The written material is first coded into meaningful pieces. For this study, descriptive coding was used initially to summarize in a word or short phrase the basic topic of a passage. Descriptive coding lead to a categorized inventory or index of data at a basic level and provided the researcher with an organizational grasp of the content (Saldaña, 2009). Descriptive coding also provided the foundation for second cycle coding, analysis, and interpretation (Saldaña, 2009).

The second phase refers to the identification and categorization of descriptors and components (Leininger \& McFarland, 2006). The data are coded and classified as related to the domain of inquiry or questions under study. Emic descriptors are studied within
\end{abstract}


context and for similarities and differences. Recurrent components are studied for their meanings (Leininger \& McFarland, 2006).

The third phase includes the identification of recurrent patterns from the data as derived from phases one and two (Leininger \& McFarland, 2006). Data are scrutinized to discover the saturation of ideas and recurrent patterns of similar or different meanings, expressions, structural forms, interpretations, or explanations of data related to the domain of inquiry. Data were examined to show patterning with respect to meanings-incontext and to further the credibility and confirmation of findings (Leininger \& McFarland, 2006).

The fourth and final phase of data analysis presents the analytic description and identification of themes. The themes whether major or minor served to produce a narrative along with theoretical formulations (Munhall, 2007) that preserved relevant meanings and interpretations from informants (Leininger \& McFarland, 2006). In this phase, the researcher included with the narrative the development of typologies, a result that has been documented to be a more common result of ethnographic data analysis than theory generation.

The primary goal of ethnography is to provide richness of data leading to in-depth descriptive analysis of cultural phenomena. In this study, the researcher continuously strived to cover the full range of the phenomena so that the resulting interpretation entailed meaningful, in-depth understanding of the culture. The resulting product of this ethnographic research is a detailed conceptual description of healthcare provider beliefs and practices regarding pediatric pain management in the non-pediatric ED. The concepts 
explicated in this ethnography may provide a sound basis for future investigations using different methodologies for data collection and analysis (Robertson \& Boyle, 1984).

\section{Rigour/Trustworthiness}

Trustworthiness in qualitative inquiry is based on five criteria that parallel to the standards of reliability and validity (Lincoln \& Guba, 1985). The five criteria are credibility, dependability, confirmability, transferability, and authenticity (Guba \& Lincoln, 1994; Lincoln \& Guba, 1985). Credibility refers to the faithfulness to the description of the phenomenon in question. It addresses the issue of consistency between the participants' views and the researcher's representation of them (Koch \& Harrington, 1998; Ryan, et al., 2007).

Dependability refers to how clearly documented, logical, and traceable the process of research is so that others can follow the researcher's documentation of data, methods, decision, and end product and potentially arrive at comparable conclusions (Ryan et al., 2007; Tobin \& Begley, 2004). Confirmability refers to the congruence between two or more independent people about the data's accuracy, relevance, or meaning (Tobin \& Begley, 2004). Transferability refers to whether or not findings can be applied outside the context of the study situation (Ryan et al., 2007). Authenticity refers to the extent to which the researchers faithfully show a range of different realities (Tobin \& Begley, 2004).

In order to meet the five criteria discussed, the following strategies were implemented in this study. To help ensure credibility of the data the researcher utilized prolonged engagement, audit trails, triangulation, and the use of a reflexive journal. Transferability and authenticity was accomplished through thick description of observed 
clinicians' pediatric procedural pain management practices, inclusion of numerous quotes, and slices of data representing the emic point of view from. Dependability and confirmability was attained through the use of audit trail, peer debriefing, member checking and triangulation of data.

\section{Participant Observation}

Participant observation refers to immersion of the researcher into a specified setting to closely observe activity and develop a rapport with the people in their natural social environment (Fetterman, 1998). The intent behind this close involvement and association is to generate data through watching and listening to what people naturally do and say and then ask a group member to explain its meaning to the researcher or participate in and observe activities to test the results of what the researcher has been told (Roper \& Shapira, 2000). Participant observation is a method used to concentrate on behaviors and the settings and circumstances in which the behaviors are seen (Spradley, 1980). This required observations of activities, and occasionally, active participation in such activities or functions.

\section{Prolonged Engagement}

In applied settings, participant observation is often spread out over an extended time. The acquisition of ethnographic knowledge is a cyclical process obtained through long term contact with the people under study. The researcher was immersed in the ED community for 5 months. Lincoln and Guba (1985) point out that prolonged engagement provides the scope and is an important step to establishing credibility. 


\section{Thick Description}

Thick description refers to rich, thorough, and vivid description of the non-pediatric emergency department, the clinicians who participated in the study, and the experiences and processes of pediatric procedural pain management observed during the inquiry (Polit \& Beck, 2008). Through the use of thick description the particularities of the setting are drawn (Brewer, 2000). To achieve this criterion, the researcher used specific examples and provided sufficient detail about the local situation and events to permit judgments by the reader about contextual similarity (Denzin \& Lincoln, 2003).

\section{Reflexive Journal}

As previously discussed, the use of a field journal or reflexive journal provided an introspective record of fieldwork by the researcher which was used to help to take into account personal biases and feelings to understand the influence on the research (Spradley, 1980). This served as the basis of reflexivity which was used to contextualize

the research (Brewer, 2000). Reflexivity by the researcher helped to ensure awareness of the situated meanings that data represented and conveyed this to the readers during the final write up (Brewer, 2000).

\section{Peer Debriefing}

Peer debriefing involves sessions with peers to review and explore various aspects of the inquiry (Polit \& Beck, 2008). Peer debriefing exposes the researcher to questions of others who are experienced in the method, the phenomenon under study, or both. The researcher consulted on a regular basis with Dr. Lev who has experience in qualitative research. The researcher also asked peers to review the research process to check whether the categories, themes, and interpretations of the data were appropriate. 


\section{Member Checking}

Member checking refers to the sharing of interpreted information with the participants in order to obtain feedback on the accuracy of the researchers' findings (Polit \& Beck, 2008). In this study, member checking was conducted in an ongoing way as the data were being collected. This was accomplished by deliberate probing in face-to-face discussions and sit down interviews with key informants where clarification and further explanation was provided to the researcher to ensure the researcher understood participant's meanings.

\section{Triangulation}

Triangulation refers to the use of multiple methods of data collection, the use of more than one theoretical framework, or more than one analysis method (Roper \& Shapira, 2000). For this study, methodological triangulation was achieved through the methods of participant observation, interviews, and examination of existing documents. Data source triangulation involved different clinicians, various times of the day, different days of the week, and different situations. Triangulation of unit of analysis by the researcher included data on behavior, perceptions, and interactions.

\section{Audit Trail}

The audit trail refers to the systematic collection of materials and documents which explicitly discusses decisions about the theoretical, methodological, and analytic choices throughout the study (Koch \& Harrington, 1998; Koch, 2006). An audit trail was used in order to establish dependability and confirmability of the findings of the inquiry. In this study, the researcher organized all field notes, transcripts, and memos during the development of the ethnographic record, using both a paper trail and computer file to be 
accessible to the dissertation committee members upon request. The researcher contacted and met with Dr. Lev periodically to help guide and verify the research process.

For this study, the implementations of the above aforementioned strategies were used to maintain the rigour, or trustworthiness, of this ethnographic study (Tobin \& Begley, 2004). The systematic collection and analysis of the data presented will provide a mechanism for ensuring the scientific merit of this qualitative form of research (Ryan et al., 2007).

\section{Summary}

This chapter described the rationale for choosing ethnographic research and included a discussion of the overall methods used in this study. Specific examples were included that depicted the procedures followed by the researcher to ensure the protection of human subjects both healthcare providers, and children with their families. The standards used to judge the trustworthiness of the study have also been included. 


\section{Chapter IV}

\section{Context and Informants}

Ethnography uses data from participant observation, key informants, formal and informal interviews, and examination of available related documents through purposive sampling. Ethnography is premised on the study of social meaning and activities of individuals and groups in a particular setting. This chapter describes the setting where the study took place, the structure of the ED environment, and introduces the key informants through summaries of the group's demographic information. This chapter also describes how data was managed throughout the process of analysis.

\section{Ethical Considerations}

Approval to conduct the study was obtained from the university and the hospital institutional review board (IRB). Inclusion criteria of healthcare provider subjects in this research study included any healthcare provider registered nurse, licensed practical nurse, advanced practice nurse, physician assistant, medical technician, nursing assistant, and physician in the emergency department who provided for or managed pediatric pain before, during, and after a procedure. Exclusion criteria included any nursing or ancillary staff that was floated to the emergency unit and was not part of the normal staff matrix. Once approval was granted the researcher met with the emergency department educator who was the main contact person for the unit and a member of the hospital IRB. The researcher offered to attend staff meetings to present the project to the nursing and medical staff; however the educator shared that the meetings were poorly attended. The ED educator sent an announcement via the emergency unit electronic mail system 
notifying staff of the researcher's presence on the unit to conduct a study and highlighted that staff may choose to participate or not participate in the project.

Prior to starting focused observations, the researcher spent time to become familiarized with the unit, and made introductions in the field approaching staff members and answering questions about the study. Most introductions were at the personal level and consent forms were in the possession of the researcher at all times during the participant observation period. Initial visits in the field consisted of constantly obtaining consent from ED staff as potentially new participants entered the situation they were requested to read the consent forms, and if interested sign and return the form to the researcher. Consent forms were disseminated during these casual encounters of the observation period in the emergency unit (Appendix D). Staff was informed that the researcher was carrying out a research study and needed to spend some time observing how healthcare providers managed procedural pain in children. During these encounters it was made clear that no one was obliged to participate and that all the information would be considered confidential. For participants who consented to participate, a copy of the consent form was made and returned to the participant in their unit mailbox.

Patient criteria involved observations of children 2 to 8 -years of age who were designated an emergency severity index of 3, 4, or 5. Patients were identified by the researcher from the emergency unit computer system. Parent and child were approached by the researcher either in the triage waiting area or when they were waiting in an assigned emergency unit room. Parents were told that the researcher was conducting a study to better understand how healthcare providers evaluated and treated a child's pain during procedures in the emergency department setting. Consent would allow the 
researcher to be in the room when a healthcare provider who consented was present and allowed the researcher to review over the patient's emergency unit chart related to the procedures performed, type and amount of analgesic prescribed and administered, and notes specific to the patient's pain (Appendix E). A copy of the consent form was given for the parents to review while the researcher stepped away to the staff work station. Prior to consent, the researcher asked if there were any questions related to the study. If a child presented to the unit with both parents the mother in all cases signed the consent form. A copy of the consent form was given to the parent and placed on the patient's chart.

\section{Historical and Sociocultural Context of Research}

The emergency department (ED) that served as the main research site was located in Central New Jersey. Fellowship was a 287 licensed private, not-for-profit acute care inpatient bed community hospital. The area surrounding the hospital included well maintained and manicured neighborhoods with middle-income housing, open fields of land and wildflowers, a local public high school, and parks and recreation area. A few miles from the hospital there were multiple commercial businesses and shopping districts. Over $80 \%$ of the population in the area was white, less than $10 \%$ of the population black, $5 \%$ of Hispanic origin, and 3\% of Asian descent. The median family income for this area was approximately $\$ 66,986$. The characteristics attributed to the hospital and surrounding area such as size and location have been altered to protect the identity of the hospital.

Fellowship Hospital's ED serviced patients from a six-county area with a population of 650,000 individuals, this placed Fellowship as one of the busiest emergency departments in the region. Fellowship Hospital admitted 53, 000 people through the ED 
in 2010 , including 8,000 pediatric patients. The ED had a total of 27 beds, 21 beds located in the main ED, and six beds allocated for the prompt care emergency section. The nursing staff for the main emergency department and the prompt care areas had set nurses, in that nurses in the main ED did not float to prompt care, if a nurse wanted overtime they could sign up. If a nurse from the ED wanted overtime, they signed up for the prompt care and prompt care nurses could sign up for time in the main. The majority of ED staff including nurses, mid-levels, physicians, technicians and registrars were Caucasian.

The ED had three focal areas triage waiting area, prompt care, and the main, at full staff each area functioned as separate units. The three areas remained in constant communication through the department's state of the art computer system. The department was heavily reliant on computer technology so much that each nurse, physician, mid-level, technician, and registrar had access to a computer. During a shift each physician, nurse, and mid-level had designated computers for their zones or areas to which they were assigned. Computer screens listed all the patients in the department and were constantly updated as patients signed-in or were discharged from the department. In the main ED there were two large flat screens mounted high on the walls and placed on opposing ends of the work station for anyone to see who a patient was, where they were, what the chief complaint was, and the acuity level assigned to that individual. The high visibility of patient information on the two screens required that the patient's initials were used on the monitors and not the individual's full names. The prompt care area was open 16 hours a day from 9 a.m. to 1 a.m. The emergency department was available to deliver care 24 hours a day, 365 days a year. 


\section{Data Management and Analysis}

In this ethnography, the objective was to incorporate multiple sources of data and triangulate one source of information against another to strip away alternative explanations and understand the patterns behind behaviors, clarify data obtained from observations, and receive feedback from informants about what was happening or why things occurred (Roper \& Shapira, 2000).

\section{Participant Observation}

The majority of data collection was through participant observation. Data were collected from March 2011 to July 2011 in Fellowship's general emergency department. Over 100 hours of observation were completed on the emergency unit. The emergency unit serviced the inhabitants of the local community as well as the surrounding areas. The average age of the ED patient who visited during the observation period ages ranged from 60 to 70 years and 40 to 50 years. The frequency of patients seen in the ED by age range is summarized, refer to Appendix F. The research was centered on healthcare providers' treatment of children 2 to 8 -years of age undergoing procedures. The researcher observed ED operations with a particular focus on 44 pediatric procedural interactions with 27 White, Black, Hispanic, Asian, and Middle Eastern healthcare providers (13 nurses, two nurse practitioners, three physician assistants, five technicians, and four physicians). With the approval of the study from Fellowship's Institutional Review Board (IRB), the hospital requested that the researcher wear business casual clothes with no lab coat to not confuse the patients or staff as to the role of the researcher. The ED was a closed off unit and required special badge access for entry. A name tag was issued by the hospital with the labeled position of researcher in capital bold black letters. An identification and 
password were assigned to the researcher for temporary access to patients' charts on the emergency unit computer system during the length of the study.

The role of the participant observer allowed the researcher to become a temporary member of the ED team and submerge into the activities of the department in order to observe what was done and hear what was said. As a temporary member of the ED team, the researcher assisted the staff by directing patient and families to rooms, bringing patients questions and concerns to the providers, and locating and conveying where staff members were when asked. During the study, participants were observed continuously during their interactions with children who presented to the department. The role of observer as participant was also adopted whereby the researcher shadowed the participant and acted primarily as an observer (Brewer, 2000; Roper \& Shapira, 2000). The researcher shadowed participants during examination of patients injuries, removal of foreign bodies, application of medication and assistive devices such as sugar tong splints, slings, crutches, and before, during, and after needle procedures such as intramuscular (IM) injections, intravenous (IV) starts, blood draws, nerve blocks, and suturing.

Observation sessions in the ED varied from 4 to 7 hours three times per week at varying times and days during the study period. A majority of observations were conducted in the prompt care area of the emergency unit as children visited this area of the ED in a more predictable pattern. The data from these observations were documented contemporaneously in a 9 X 6 stenographer pad, expanded on a word document within 24 hours of observation and transferred into the Ethnograph 6.0 database software program (Seidel, 1998). 
Observations at the start of the study focused on understanding the dynamics of the department, the type and age of patients treated, the personnel involved, the use of color coded scrubs to identify a participant's status, familiarity with the staff and department computer system. Field notes toward the middle and end part of the study recorded comments and actions of participants before, during, and after a procedural related pain with children.

\section{Field Notes}

Ethnographic field notes served as the main data source. The field notes contained the date, days, times, weather, names, job titles, and activities of participants and informants. Preliminary field notes were written in a steno pad and expanded descriptions were added within 24 hours of observation this was due to the fact that some observations ended at 2 a.m. During observations of pain related procedures brief notes were written contemporaneously and following the event, the researcher went into the ladies locker room or staff lounge to immediately elaborate on the details. Field notes also included contextual descriptions such as how many patients were waiting to be seen, the number of staff working, what occurred during the period of observation when there were no study cases in the department, and staff comments relating to general pain management. At the beginning and end of each observation, the total number and ages of patients in the ED at those times were also recorded.

The age of general ED patients was entered into the IBM Software Package for Social Sciences (SPSS) 20.0 where data were analyzed to show the frequency of visits by age (Appendix F). The 44 individual case studies were entered into a Microsoft Excel Spread Sheet where the age, chief complaint, pain scale score, and comments and 
behaviors of the parent, nurse, technician, mid-level, and physician were broken down, coded, and analyzed. The codes for analyzing behaviors were developed both inductively and deductively guided by the Davis observation code (Walters, Prideaux, Worley, Greenhill, \& Rolfe, 2009), the Proximal-distal model of children's coping and distress during painful medical procedures (Blount, Bunke, \& Zaff, 2000), Comfort theory and practice (Kolcaba, 2003) as well as readings from the nursing, dentistry, and medical literature (Abbe et al., 2007; Ramponi, 2009; Schechter, 1989). The codes are presented in Appendix G. The frequency of behaviors is summarized, refer to Appendix $\mathrm{H}$.

\section{Introduction to Key Informants}

The goal of purposively choosing a key informant was to find a cultural consultant during the investigation who wanted to share time and knowledge and teach the researcher about the practices and beliefs about the group. The key informants were knowledgeable of the research setting, experienced and understood the management of children in the ED, and were articulate in their ability to communicate with the researcher. Key informants provided insight into their role in the management of procedural related pain in children, each informant helped to explain subtleties of the ED culture. For instance, one informant worked strictly in the prompt care area as a technician (Graham), one informant worked in the prompt care area as a nurse (Ginelle), three informants worked evenings (Maura, Kate Lyn, \& Lizza), and one informant worked only nights in the main ED (Lain). Key informants consisted of six participants (five nurses and one emergency technician). All could speak, read, and understand English. 
During the study, one key informant was identified and agreed to sit for an interview; however after two verbal reminders, two email attempts, and one follow-up phone message the researcher did not further pursue this individual as an informant.

\section{Demographic Data Key Informants}

There were five females and one male whose ages ranged from 31 to 55-years-old $($ mean $=46)$. The years of practice ranged from two to 34 years $($ mean $=20)$ and the years working with children reflected similar responses except for one participant who worked with children as a medical assistant in a pediatric office prior to becoming a nurse, and one participant who worked on adult units prior to working in the emergency unit. Four of the participants were parents and one participant chose not to answer the question. Three of the participants identified their ethnicity as Caucasian, one of the participants American, and two participants designated themselves Hispanic. The demographics of key informants is summarized, refer to Appendix I.

\section{Interviews}

Prior to scheduling the first interview, the researcher made observations in the field and took part in friendly conversations with participants to help build rapport. Semistructured interviews were scheduled with all key informants. The focus of the interview was to openly discuss the management of procedural related pain of children in the emergency department. The preliminary semi-structured interview questions (Appendix C) were used during the first interview with Ginelle specific items were constructed based on researcher experience and the literature review. For subsequent interviews, the question format was expanded based on information gained during observations, and 
content discussed in previous interviews. The revised semi-structured questions are included see Appendix J.

Each informant was interviewed once. The interviews took place at times convenient to the participants on their days off. Two interviews took place in a quite coffee shop; four interviews took place in a conference styled room outside of the hospital which was quiet and free from interruptions. The locations were selected for informants' convenience and the researcher's safety. At the beginning of each interview, the purpose was again explained and permission to audio record was obtained (Appendix K). All informants agreed to have their interviews audio recorded. Interviews with key informants were purposefully sampled throughout the study period. Respondents were reminded that the interview would be treated as confidential. Interviews varied in length from 30 minutes to 90 minutes (average 60 minutes). Informants were asked to provide the background demographic information stated above. Field notes were made immediately after each interview to describe non-verbal communications and their contexts. Informants appeared to speak freely about their roles in the ED, the demands they encountered in their work and the strategies they used to work with children and manage their comfort. The last interview was a formal member check where the researcher discussed with the informant the themes and categories developed from the data.

Informal conversations with key informants and participant nurses, technicians, midlevels, and physicians during the observation period were used to extend the observational data and to clarify the meaning subjects themselves attributed to a situation. Informal questions focused on thoughts about procedure related pain in children, the 
influence of participants having children, interpretations of children's behavior and feelings of how the overall procedure transpired. Informal questions added to observations and served to confirm what was observed. Informal conversations were incorporated into observation field notes. Informal interviews served a dual purpose the first was to discover what participants thought and how one individual's perceptions compared with another. The second purpose was to establish and maintain a healthy rapport with the group participants' (Fetterman, 1998).

Interview transcripts were transcribed verbatim by the researcher. The researcher then reviewed the transcripts and audio recordings line by line for accuracy. Interview transcripts were then entered into the Ethnograph version 6.0 database software program (Seidel, 1998). Transcripts were constantly compared against each other to look for patterns of similarities and differences.

\section{Documents}

Information was gathered from the child's electronic emergency chart related to pain documentation, pain assessment, type of pain scale utilized, procedures performed, type and amount of analgesic prescribed and administered, and notes specific to the child's pain experiences. The researcher reviewed charts while patients were in the ED; no retrospective chart review was conducted. The review of documentation during a patient's stay confirmed or clarified discrepancies between what was done versus what was documented by participants. Other documents included hospital and emergency unit policies regarding the assessment and management of pain in children (Appendix L). Institutional policies were examined to assess the existence of pain related policies for children, to assess if pain policies for children were current or outdated, the availability of 
policy to participants, and to understand if patterns of behavior related to procedure related pain were guided by institutional policy or individual practices. Articles and documents obtained via Fellowship's intranet system provided data on the values and concerns of the overall institution. Newspaper articles from the local paper provided contextual data as to the current status of the hospital within the larger surrounding community.

\section{Reflexive Journal}

Throughout the data collection, analysis and write up of the study, the researcher maintained a reflexive journal in addition to field notes. The journal was used as a therapeutic means to reflect on what was seen, feelings related to the research process, the venting of feelings toward participants and a reflection of the researcher's values and assumptions. The journal was also used to construct thoughts, and ground the researcher. Information about methodological decisions and reasons of making them were also recorded. The goal was to minimize researcher bias and ensure that the data collected was an accurate representation of the participants' worldview (Appendix M).

\section{Data Analysis}

Data analysis followed the procedure developed by Leininger and McFarland (2006). Data were simultaneously coded and analyzed as it was collected. ED observational field notes, transcribed formal interviews, informal conversations, documents, and reflexive journals were used as the basis of analysis. Data analysis began with descriptive coding, in which a word or short phrase was highlighted and used to summarize the basic topic of a passage. Descriptive coding developed a very basic foundation to organize data into units of information. Secondary codes were then developed to address specific research 
questions such as behaviors, participant perception, unwritten rules, practices, beliefs, and tools. Code lists were printed out various times during the analysis of data. Changes made to codes and reasons for the changes were written in reflexive memos. Initially, memos were recorded within the software program, but the program did not allow the researcher to diagram within the memo function memos were then recorded on word documents.

All memos and codes with complete descriptions of their definitions and properties were printed. A continuous process of reading, and re-reading all the field notes, transcripts of semi-structured interviews, documents, and reflexive memos facilitated comparisons among units of analysis for similarities and differences. Similar thoughts, events, and incidents were labeled and grouped to form categories. As categories surfaced, each category was then compared with units of information for each observation and interview. Reflexive memos representative of the researcher's thoughts about data, codes, and categories was ongoing. Descriptive measures were also applied to summarize and describe the data from the participants procedure related pain behaviors and involved frequency distributions. When no new patterns emerged data collection and analysis were considered complete.

\section{Summary}

In this chapter the group of participants and methods of sampling those informants were introduced. Demographic related information was described. Purposive sampling methods as well as the theoretical sampling methods used in the study were also presented. 


\section{Chapter V}

\section{Description and Discussion of Themes}

Ethnography is a qualitative method that emphasizes the concept of culture.

Ethnography is an inquiry into the lives of people to understand their ways of living, and the meanings they attach to activities, events, and knowledge. The goal of ethnographic research is to translate and interpret the behavior of participants into meanings.

\section{Major Themes and Categories}

The context of healthcare provider evaluation and treatment of pediatric procedural pain in a non-pediatric emergency setting are depicted in the following sections. Each circumstance will be discussed in detail below, using specific examples from semistructured interviews with informants, participant observation, and document analysis. The examples were chosen because they represent an overriding theme identified by informants.

\section{Assessment}

Pain assessment appeared to have a high priority on the emergency unit. The assessment of pain was an ongoing process from the time the patient presented to the emergency unit until discharge. Pain assessment and documentation involved a pain score, the location or site of the pain, pain duration, frequency, characteristics, factors that aggravated pain and factors that alleviated pain.

When is assessment done? In the triage area, the Wong-Baker FACES Scale was the pain intensity tool frequently identified by participants for use in children. The tool adhered to the right side wall panel next to the patient intake seat that made it easy for patients to see and point to the scale. In the prompt care area and the main department a 
laminated $8.5 \times 11$ inch pain intensity tool sheet was adhered to the wall a few feet from the patient stretcher (Refer to Appendix N). In greater than $60 \%$ of cases $(n=29)$ a pain intensity score was documented on arrival of the patient to triage by a nurse. In over $70 \%$ of cases $(n=34)$ a more in-depth pain assessment was documented by the bedside nurse after the patient was assigned to an emergency room. Lain described the use of pain assessment tools in the department:

In triage we have the post it [Wong-Baker FACES Scale], it's right there so we go by that when we show the faces there, in the back they have the cards... I think they have the same card that's in triage, I think they have it in the back as well that they can take it to the bedside. No [I have not seen people use that] to be honest with you, No.

In triage and at the bedside, documentation of a pain intensity score was used to measure the presence of pain from illness or injury on admission to the emergency unit. The documentation process followed Fellowship's policy on the management of the patient with pain:

Upon admission to the outpatient area [emergency unit], the presence of pain will be assessed. When pain is present the admission assessment will include the location/site of pain, pain intensity, pain duration/frequency, characteristics, aggravating factors, alleviating factors and intervention. (Hospital Policy Document)

In general, the assessment and evaluation of a child's pain was a shared responsibility of the technician, nurse, and physician. The technician had a more informal role in the assessment of pain as they were not primarily responsible for the assessment and documentation of pain findings; however technicians on occasion did ask children to subjectively rate their pain and relay pain findings to nurses. This is illustrated during an informal conversation with one of the participants and observations:

Researcher: Do you assess pain as a tech?

Technician: Well kind of, the triage nurse does their basic assessment and if they 
rate pain as 0 I do not reassess. Sometimes, I reassess their pain if the patients were anxious initially to get a better indicator of pain. (Field notes, 04/13)

I observed the technician tend to a 6-year-old girl with a fractured arm, attempting To obtain vitals. The child and mother point to the uninjured arm that is not in a sling. The blood pressure cuff starts to inflate. The girl says, "this is hurting" the blood pressure cuff beeps. The technician re-adjusts the cuff, "let's try one more time and see what happens". She re-sets the monitor and pushes the start button. The tech leans down a bit and asks, "what's your pain from 1-10, how bad hurt?" I am not sure what pain she was referring to was it the pain from the cuff or did she want to know if there was pain from the fractured arm. The young girl stated, "It doesn't hurt that bad right now, just a little." The technician and patient's mother asked again reinforcing that she assign a number to her pain, "so what number 6, 7?". (Field Notes, 04/25)

In the above observation, the technician did attempt to assess pain, but overlooked the fact that the child did not understand the concept of assigning a number to reflect the intensity of pain. No explanation was provided to the child as to how the numbers related to pain intensity. One informant illustrated concern about the limitations of using a pain measurement tool with children:

We use the FACES because that's what they [children] can pretty much relate to I think. As far as the numbers I don't think some of them understand the severity of pain. I don't think that most kids understand the FACES. They sit there and they look at it and they're like (using a puzzled face). The mother has to coach them into saying how do you feel, this is if you're not having pain it's this face, if you're having pain it's this face, so they don't really have a full understanding to what they're pointing to. (Lain)

Physicians rarely assessed a child's pain using a pain intensity tool. The physicians and mid-levels examination were focused on identifying the cause and extent of injury or illness and the presence and location of pain in order to facilitate the ordering of therapeutic and diagnostic procedures. If pain scores were documented by physicians, nurse practitioners, or physician assistants, pain scores were taken from the triage nurses documentation of pain. The field note below describes the examination of a child with a 
potential orthopedic injury. The physician assistant assessment focused on the extent of

the injury, but the child was not asked to rate the intensity of their pain.

An 8-year-old male has pain documented in triage 8 aching and throbbing. The midlevel (physician assistant)enters the room.

Mid-level: What happened?

Mom: He fell off the monkey bars.

Mid-level: Were you climbing on top, or just going across?

Mom: We didn't see he just told us that he fell and hurt his arm.

The mid-level looks at the boy and leans in, "tell me where it hurts? Okay, let me examine."

The mid-level palpates the back of his neck and front of his chest, palpates a small section of his left lower arm starting from the elbow moving slowly down to the wrist. "Does it hurt? Yeah, it's very swollen down here." The mid-level turns and looks at the parents "can we give him some Motrin for the pain?" (Field Notes, 04/16)

The mid-level did not assess for the intensity of pain in the child, but did order some type of analgesic to address the patients potential pain associated with the injury.

\section{Whose assessment is evaluated-patient, parent, healthcare provider? In a}

majority of cases $(n=42)$ participants assessed for the presence and location of pain. In most cases, neither the parent nor the child were asked to rate the intensity of pain by any healthcare provider, but a pain intensity score was documented on the chart. Pain intensity scores appeared to be more reflective of the nurses' opinion about how much pain the child experienced. In $75 \%$ of cases $(n=33)$ in triage and $52 \%$ of cases $(n=23)$ at the bedside no specific pain tool was identified. The following observation depicts how one participant assigned a pain intensity score:

A 3-year-old was brought into the ED after tripping over his dog two days ago and presented with a visible limp. The researcher reviewed over the initial triage documentation of pain and was puzzled by the letters UN.

Researcher: What does UN mean?

Nurse: Unable, but I see no crying, no complaint; I'm going to put 2 when I look at his face. (Field Notes, 04/24) 
Some participants' appeared to use the FACES scale as a behavioral tool to measure the pain intensity of a child. The measurement of pain in relation to the behavior of the child was best illustrated by Lizza:

Pretty much observe, you know and you also know the injury, you know if you see an injury a deformity, or you know a bowed arm you know they're in pain, do you really need a scale? The kid has an obvious fracture, you know there's a big open wound, you know that hurts the kid is crying inconsolably, like that child with the toothache, did I need a Wong-Baker, NO, she was 8, did I...she's hysterical crying for an hour and a half, do I have to say to her, gee, how bad does that hurt (laughing).

Participants seemed to base their individual perceptual awareness of a given situation as a more reliable indicator to the amount of pain experienced by a child. This awareness was dependent on decoding what was going on in each situation. Participants also attributed personal knowing drawn from inner knowledge of previous experiences with children as a reference to assessing pain. Sometimes this was attributed to experiences with one's own children, the degree of injury and the associated pain believed to accompany such an injury, or personal pain experiences from similar injuries. Graham illustrated this idea best:

Sometimes it's a vibe thing too whether you've had that injury yourself, like going through stitches, so you know what it's like to have this big gapping wound all open on you, and you see that, and it hits home for you. I know what it feels like or you could understand a person's in pain, or you could see an injury, you know there's pain, and sometimes you can't even imagine, it's a vibe.

Some participants were more likely to advocate for pediatric patients who they perceived to be in a lot of pain versus patients who said they were experiencing high levels of pain. The subjective nature of a child's pain was considered secondary to the healthcare providers overall pain assessment. Pain intensity scores of children were never assessed or evaluated before, during, or after any needle work procedure such as blood draws, intravenous access, or intramuscular injections. 
Analysis of the data revealed that assessment of pediatric pain was dependent on the nurses' perception of the patient's pain as no pain assessment tool was regularly used to obtain the patients' pain intensity score. Participant perceptions were based on a hierarchy of painful procedures, and pain behaviors of the child.

Hierarchy of Painful Procedures. Management of pediatric procedural pain was identified by participants to be dependent on the type of procedure performed, and the degree of illness or injury. Two categories, high end and low end procedures, are defined. High end refers to procedures that require immediate pain management due to severity of injury and provider perceived concurrent pain, such as a large gapping laceration, displaced or angulated fracture, intubation, and intraosseous line placement. Low end refers to procedures that are conducted on a routine basis and that are perceived by the provider to be benign such as blood work, intravenous venous line insertion, injections, straight catheterization, and the placement of sutures or staples.

The priority of controlling procedural pain was not easily discernable to participants because pain management was dependent on the invasiveness of the procedure. This is illustrated by Ginelle:

That's tough because it depends on what you are doing, if it's a kid with a fracture or you know they're in a lot of pain because their arm is broken and the orthopedic is coming to set it; then you want them to be out of pain as quickly as possible to have them calm so that when the orthopedic gets there he can set the arm.

Lizza referred to the urgency of providing pain relief citing fractures and burns as major points of concern.

If you can see there's an obvious injury, a deformity, or a fracture, you know you need to get that kid something for pain. We had an 8-year-old who was burned very badly, and we did give IV Morphine to that child and made a big difference, it was a serious burn. He had to be actually helicoptered out to the burn center and he was burned over $50 \%$ of his body. He was in a lot of pain and wailing and everybody was 
in an uproar, you know it was quite a scene in the ER that day so our first priority was to get an IV in this child, get a set of vital signs, get some fluids running, and get him out of pain immediately.

When asked about procedural pain many of the participants associated it with setting broken bones, severe lacerations and using conscious sedation. Participants did not associate blood draws and IV starts with procedural pain. This is captured best during an informal conversation:

Participant: What can I tell you?

Researcher: What do you do for pediatric procedural pain?

Participant: We don't do many procedures. Procedures that require sedation, those kids are really sick and go to the ED.

Researcher: What about blood draws, IV's, and suturing?

Participant: Well, I guess those are procedures, but we don't do anything. [Field notes, 03/28]

Typical blood draws and IV starts were not given such a priority. Only one informant stated that they advocated for the use of a topical anesthetic, but in their example it was an adolescent who the nurse viewed to be extremely needle phobic:

Well if you get a child that says (dramatic panic) you're not going to give me a needle, are you? You're not! You don't have a needle? You're not going to put that needle? You can tell right away, and say we're going to make the needle not hurt altogether, we're going to put this special medicine on you and you're not going to feel anything, so you don't have to worry, you know those are the types of patients you're going to use it on. Doctors always will give it to me. Sure, that's fine, whatever you need go right ahead. It's not a big deal (laughing). I wish they had the time to use it on the adults, adults go through a lot. You know, EMLA cream cuts down on the sensitivity of that needle going though the skin, but we don't do that. [Lizza]

Ginelle identified other procedures outside of setting bones, and use of needles as sources for procedural pain:

Blood draws, even $x$-rays in the ER if you take them for an $x$-ray they're afraid. So any kind of testing they have to have done or any kind of medication that they have to be given, even for them to take some Tylenol if they're having fever. 
The participants varied in their interpretations as to the benefits of why certain treatments were performed while some of the providers believed that conscious sedation was needed to comfort the child others interpreted it from an injury management stand point rather than comfort. The need to have the child nice and relaxed was identified as a key variable to reduce dislocations and coincidentally helpful to manage a child's pain during the procedure. The use of conscious sedation was not seen to be a routine practice used for young children during procedures.

Questioning the Reliability of Pain Scales. While participants confirmed the presence and use of pain scales such as the Wong-Baker FACES scale and the FLACC behavioral scale on their work unit, the use of such tools were viewed as inaccurate by participants as indicators of a child's true pain.

(Laughing) I don't think they're reliable at all I think it's nonsense! We show (kids) the smiley faces, but the funniest thing is they always point to the smiley face. Kids are very funny that way. The kid could have a broken arm and I'll say, how do you feel right now, and they'll point more toward the smiley face. Where, you know, they broke their nail and they'll tell me their pain's a 10, you know an adult. I think people think if they tell us the pain is worse they'll get in faster. (Maura)

(Graham) You know when you're not going to get a real answer, you have to coerce an answer out of them, you know if it's a 10-year-old and she says my pain is 10 (he holds his hands up in the air and pretends to be texting or playing a video game), but they are steady texting on the phone your pain's not that bad, they're posting on Facebook and taking pictures of their injuries, your telling me your pains a 10, it not a 10. Will I put a 10? Yeah, but mentally we know its not a 10.

Conversely, one of the informants indicated the use of both the parent as well as the scale as a good indicator of pain.

They [The parents] would be the best historian, and they would kind of guide you, and lead you that she's not comfortable. I think they're [the scales] pretty good because that's what you have to use. When these kids are in pain that's just about the best; I think that's out there to assessing their pain.[Ginelle] 
Pain scales were never used for the assessment and evaluation of pain before any needle work procedure. If documentation was provided, a child's response to a procedure was mainly summed up as patient tolerated procedure well.

Overt Pain Behavior of the Child. For participants visually seeing or hearing children complain was an indication of the amount of pain experienced by a child. Participant's considered both active pain behaviors and non-active pain behaviors to sensitize themselves to the presence of a child's level of pain. Overt or active pain behaviors refer to outward displays of behavior that sensitized the provider to the presence of a child's pain. Non-active behaviors refer to the manner that a child withdraws socially and/or guards an area of injury that sensitizes the provider to the presence of pain.

(Ginelle)Trying to know what their pain levels are, or how they express pain, because they don't always say, they're not always capable of telling you it hurts here, so you have to know if they are rubbing their head that it is because their head hurts even though they are not able to tell you that. You know because I had a 2year-old with a tumor, a brain tumor, and she never said her head hurt. She would just always hold her head when it hurt.

A majority of participants assessed both cry and body language as indicators of overt pain in children. A common overt pain behavior perceived by participants was the use of cry. Other examples of overt pain behaviors involved facial grimacing, wincing, body contortion such as pulling legs toward the abdomen, twisting and turning from the source of pain, screaming, hollering, and hyperventilation. This was illustrated by this participant:

You know kids generally not going to tell you that it hurts they just start crying I just go by their body language and how they're acting also by their injury. If you can see that there's an obvious injury, a deformity, or a fracture, you know you need to get that kid something for pain, then you know once you're finished whatever you needed and they're still crying there's something wrong, kids have a way of body language 
of letting you know there's something wrong. They're still crying, they're not consolable. (Field Notes, 04/26).

Some participants further expressed that overt pain behaviors provided a limited parameter in the assessment of a child's pain. The use of cry by children was viewed as highly variable and an expression of other emotions such as fear and anxiety related to stranger danger and anticipated separation from mom and dad. The assessment of nonactive pain behaviors such as a child's decreased socialization with caregivers, and withdrawn behavior were ominous indicators of pain.

(Maura) I mean they cry anyway they cry when they see you, so you can't go by crying. I worry the more, the more quiet they are, they're normally withdrawn. They don't even want you to come near them.

(Kate Lyn) There's a difference between a kid crying, and a kid moaning, crying, thrashing, you know, a lot of times they become very combative, that kid's in pain. They don't want you to come near them, but they're very protective of whatever hurts.

Participants stated the importance in assessment of behavior as an indication of pain in pediatric patients, but rarely interpreted overt pain behaviors during procedures as a priority. Kicking, screaming, shouting, crying, and withdrawing from a painful stimulus were expected behaviors from children during certain procedures.

Timeline from assessment to prescription. The timeline from pain assessment to prescription varied. Simple comfort measures such as giving an ice pack for an obvious injury were sometimes provided by the triage nurse. While physicians and mid-levels were observed to ask about pain and prescribe analgesics such as Tylenol or Motrin, the delivery of medication was highly dependent on nurse availability.

An exception to the delay of medication administration occurred if a participant identified and conveyed verbally for the nurse to administer pain medication or if the 
nurse verbalized to the physician or mid-level that a patient was in a lot of pain. Two exceptions identified included: 1) if the physician or mid-level was the first to examine the patient and verbally told the nurse to give a medication for a patient the medication was administered, or 2) if a nurse identified that a patient appeared to be in a lot of pain and verbalized the assessment to the physician or mid-level an order was given and the medication promptly administered.

A 7-year-old is brought in by wheelchair to prompt care. He is assisted to the stretcher by his mother who picks him up. The technician is the first person to greet the patient, "what happened?" The boy appears very upset crying and sobbing the boy explains how he tripped and fell down five steps coming down the slide ladder and landed over his arm when running after his cousin. The technician stands at the right foot side of the stretcher, "we'll have to take an $x$-ray, the nurse will be right in." The nurse enters the room, "what happened?" The boy retells the story. While the boy is speaking the nurse walks to the side of the stretcher closest to the boy, "where does it hurt?" The nurse looks at the arm then goes to the work station and shares with the mid-level (physician assistant), "that boy is in a lot of pain." the midlevel asks about allergies, weight, and orders Motrin. The nurse prepares the medicine gives the medicine cup to the mother and walks away. The mother goes to thank the nurse, but the nurse has already left the room.(Field Notes, 05/20)

In the above case, the patient was medicated prior to being seen by the mid-level and prior to any physical manipulation of the injured arm. Verbal communication of the patient's pain by the nurse to the mid-level facilitated the administration of analgesics.

How is information on assessment communicated? In the emergency department all documentation was completed on the computerized system, the charting system was standardized and allowed staff the choice to document pain management. The computer system highlighted abnormal values such as out of normal range vital signs or elevated pain scores. Verbal communication among participants regarding pain in a child was reserved for patients perceived to be in a lot of pain by the emergency unit staff. Otherwise the communication of pain was entered in the computer documentation system 
and available to be accessed with the click of a computer key. One participant pointed out the differences between the treatment of an adult and pediatric patient with the same injury:

If a kid comes in with a broken arm, they're always whisking them off to $x$-ray and you know not thinking about [the pain] they're always afraid to medicate kids...I don't know they're crying, the mother's upset, and they just seem to under medicate these kids, where I think these kids with the broken arm or deformities or dislocations require as much as an adult to sometimes get out of pain. They seem to forget that a lot of the times with kids.

Researcher: So, if there is an adult who comes in with a broken arm, what do they do for them?

Lizza: They have a dislocated elbow they'll put a line in and give Morphine before the patient even goes to $x$-ray. The kid always seems to be whisked off to $x$-ray and then, oh yeah, we gotta give'em something for pain after he's suffered for 45 minutes.

\section{Summary of Assessment}

Procedure related pain was rarely measured and documented. The measurement of pain in the ED was designed to trend painful conditions related to illness or injury when patients presented to the department. The measurement of pain intensity appeared to represent the health care providers' interpretation of a child's pain. Health care provider interpretation of a child's pain was influenced by health care providers concerns for reliability of child's self-report, hierarchy of painful procedure, and overt behavior by the child. Timeliness from assessment to administration appeared to be dependent on nurse availability and how healthcare providers communicated pain concerns.

\section{Procedural Pain}

On the emergency unit, the most common procedural related pain included the application of sutures, venipuncture, and peripheral intravenous catheterization. The management of children during pain related procedures differed from pain related to 
disease and other etiology in two ways; that is, the use of physically restrictive devices and the use of experts.

Restraints. Unlike positioning where a child's comfort assumes primacy, the goal of restraint was to safely allow for ease and accessibility to the area in need of attention or repair. The use of physical restraint was used for children who were considered unable to keep still, too young to understand any explanation, and for children who were considered inconsolable and combative. Physical restraint provided the healthcare provider with control over the situation to maintain the child in a safe position in order to conduct an invasive procedure. Some of the younger children were referred to by the staff as, "small and mighty". The staff power required to restrain a young child ranged from two to three people. Holding down a child or physical restraint was a standard procedural technique used in the ED. For participants it was seen as a necessary component of care.

(Graham) For the most part most of them [parents] understand, it has to be done, and if we hold them down they're gonna get a better result. Some of the mothers or fathers they don't understand the screaming escalates and they think it's from what we're doing, but really it's from the child or anybody in general being restrained and can't move, and it escalates which in turn gets the kid even more riled up.

(Ginelle) With regard to the younger children 2, I mean you really can't tell them too much. I'm sure some of them understand but at that age they're just screaming so it's just better to get it done.

While the practice of restraint was commonly used in the department for the care of children during procedures, participants did not document the use of any restraints. As part of the socialization process of the researcher, educators at Fellowship included a mini-orientation informational session designed for students, interns, and temporary staff, both written and verbal information was given regarding the policy of restraint use in the 
institution. The following definition was from the orientation information guide: (Refer to Appendix O)

A restraint is defined as any manual method of physically restricting a person's freedom of movement, or normal access to his or her body, material, or equipment, attached or adjacent to the patient's body that he/she cannot easily remove.

The use of white linens would be wrapped around the child and tucked under the child's body to use as a counter weight. The use of either or both, parents and healthcare providers to hold the head, legs, and feet were common practice. Certain cases required the use of three people to assist in the restraint of a child. Depending on the severity of the injury, a child could be restrained in a position for less than a minute or as long as 40 minutes. One of the informants illustrated the challenges to using restraints on children:

(Lain) I think it's more traumatizing and it does induce pain because the patient is moving about trying to get loose, screaming, hollering, contracting muscles. Let me give you an example before I became a nurse my child, my oldest one, needed stitches and they put him in a papoose, that was traumatizing for me as a parent. I mean now that I'm a nurse I understand why, but it is traumatizing for both parties, because the parent is saying oh my god my child's in pain I can't do anything for them, and the little person is also like you're suppose to help me.

The frequency of using physically restrictive devices during suturing was considered routine and common place that participants took for granted the need to effectively prepare the family for the procedure. The following example best illustrates this dynamic:

A 2-year-old is brought in for a 2.5 centimeter laceration to the right forehead. The mother approaches the work station and speaks with the mid-level about getting her child a drink.

Mid-Level (physician assistant): Well, if we are going to tie her down she might get upset and make herself nauseous.

The mother's eyes widen and her mouth opens as she turns away and says, "tied down?" She lifts her hand to her head in disbelief. The mid-level rises out of the chair and walks quickly toward the patient room to clarify for the parents and family what was meant by tie down. (Field Note, 06/11) 
Experts Performing Invasive Procedures. Emergency nurses, and technicians

varied in their degree of experience and comfort performing invasive procedures on young children. For instances where a procedure was needed and the emergency nurse or technician felt limited in their ability or was uncomfortable to perform the task an expert was called in to assist with the situation.

(Maura)You know what we do for that, we get the best person in there. They'll stop (what they're doing) and help with the kid.

I asked the prompt care technician, "how come you asked the other tech" to come into the room and draw blood work on your patient?

Technician: I just wanted her too, when I heard she's [the patient] a fighter, if she couldn't do it then I would have, but I couldn't deal with that right now. (Pause) I can draw bloods and do that but E. is good. (Field Note, 04/25)

For participants who were considered experts, there was an increased pressure to

succeed in the task. This was illustrated best by this informant:

I think I do a pretty good job. I usually only have to stick'em once, umm luckily have had the experience under my belt that I don't get intimidated by a child. I've put an IV in a 2 day old already in the foot cause I had too, and I felt a lot of pressure because I knew the kid needed IV antibiotics and they came and got me and I was like, oh I got to get this line in, you know I felt the pressure of it this has to get in this kid, this isn't an option for me so there's stress and pressure. (Lizza)

Participants who dealt with children on a regular basis in the department were sought out by their peers and the constant referral to attend to children developed their pediatric skills further. Reliance on the use of experts by some participants perpetuated the lack of comfort and general uneasiness of working with children.

I just think once you become seasoned and start becoming good and caring about your job... if you care about what you do...its gonna show in every aspect of how you treat a patient especially a pediatric patient. I mean you have to have really good skills, you have to be a people person, you have to know intuitively what to do in a baffling situation. It just comes to you without having to look for it. Your first response is the right one...it's not something you have to think $2+2$ is 4 it's not like that, it's you see something and you become it...It's very fluid, I mean you become very fluid in what you do, and I think after awhile you just intuitively know what to 
do...I mean sometimes there's no time to think, some people have to think about things, and there's people I imagine you see that have to think too much. (Graham)

\section{Summary of Procedural Pain}

The participants in this study identified the use of physical restraint and expert technical skill as necessary components for the management of children during painful procedures. The use of "holding" or physical restraint was a common practice used by participant, but rarely documented on the medical record. The use of experts was viewed by some participants to contribute to the continued uneasiness of managing pediatric patients in the ED.

\section{Treatment of Pain}

Participants used a combination of pharmacological therapy, and non-

pharmacological therapy to manage a child during procedures. The following case study exemplifies the treatment of child who presented to the ED in need of sutures to the head.

Case: A 3-year-old is brought to the emergency department for a laceration on the chin. The boy clings to his mom as they walk into the prompt care area. The mom sits in the chair with the boy on her lap. The mid-level approached the mother, "We are going to start this, the L.E.T. to help with the pain. Can I look?" The mid-level stands to the left and attempts to examine the injury. The mother attempts to distract the boy with his Lightening McQueen toy matchbox car, but the boy turns his head to the right, in the opposite direction and starts to cry. As we walk away from the mother and boy the mid-level (physician assistant) says, "He's going to fight, this will not be good." After 3 doses of L.E.T., I walked into the room with the mid-level to do the sutures on the chin laceration. The technician was in the room and the boy was placed on the stretcher.

The technician started to wrap the boy in a sheet. The technician took a folded sheet placed it behind the boy and brought the sides toward the front and folded it over the boy's body. She tucked the loose side pieces under the boy's sides. The boy was crying before, but started screaming and fighting, pushing up on his legs to break free from the sheet. The mom backed away from the stretcher while the staff was securing the child, mom threw her arms up in the air while standing off to the side and looking at her son, "You just tell me what you want me to do." The technician in a calm and flat voice said, "I will hold his head, you hold his body." The nurse enters from the doorway, "You need help?" The mid-level and tech look at him, "YES.". The nurse comes in and takes a bed sheet and folds it over and over and 
places it underneath the child's head, but the sheet is too thick. So the nurse removes the sheet unfolded it once or twice and reinserts the sheet under the child's shoulders. The head is now slightly hyper-extended. The mid-level moves to the right side of the stretcher she takes a piece of gauze and applies direct pressure on the chin laceration. She says to the mom, "It's numb, you see how it's white, the medicine is working." The nurse holds the boy's head midline. The tech is to the left of the stretcher holding down the boy's pelvis, and the mom holds his feet. The boy continues to thrash around the stretcher. The mid-level is preparing the suture material and says, "That's good, hopefully he'll tire out before I get there." "That's wishful thinking" retorts the nurse. The boy continues screaming and fighting, "NOOOOOOO! NOOOOOO! STOP IT!" His body is the color of a cherry. He starts to gag on his on saliva and mucus. The nurse continues to hold the boys head midline and stable in a moderate voice he says, "It's alright buddy, just calm down, calm down." The mid-level stood there waiting for an opportune time to insert the first stitch, but the boy was violently fighting back. The nurse and mid-level are making small talk with the mother, the boy continues to scream. The mid-level says, "If you just kept still, and stop moving things would go faster, and of course you don't understand that concept." The boy starts to cough in the mid-level's face, she backs away slightly every now and then. "He'll be tired tonight" said the nurse. Don't let him go, get Bacitracin and a band-aid," directed the mid-level. Once the ointment and band-aid were on the staff released the boy from his restraints, "Donnne, Donnne," he reached for his mother and she reached for him. His entire body and head were bright red. His mother went to wipe his tears and push his hair away from his face. His blonde hair contrasted with the bright redness of his skin. He stopped crying, his mom "Shhhh, shhhh" in gentle tones "all done." The boy did not cry anymore he looked behind him, above him, to the side of him, and maintained a watchful eye that no one was approaching him anymore. The procedure start time 19:10. Procedure end time 19:44. (Field Notes,04/18).

Participants were quick to point out pharmacological therapies as a way to manage children's pain, but other treatments such as providing ice packs, application of devices such as a sling or splint were also implemented. The uses of such medical devices were conceptualized to represent the more technical aspects of managing pain.

Pharmacological. All participants identified pharmacological treatments such as the use of narcotics, oral and topical analgesics as the gold standard for the management of procedural pain. The use of the lidocaine, epinephrine, and tetraciane (L.E.T) mixture gel was the most regularly used treatment prior to suturing any individual, young child or 
adult, who presented to the emergency department. Graham summed up the use of L.E.T gel application:

You see that we are big time in with the cuts and everything, we're using the L.E.T gel application. Is that as effective as a straight Lidocaine injection? Probably not. We try to kind of wing it, I mean cause you can see it working, the skin starts blanching, so you kind of can see it visually happening. You have no sure shot way of knowing if it's the whole area affected by the L.E.T gel... I think you could say about pediatric either [they're] going to be pain tolerant or you're not because there are some children who go through the procedure magnificently, they don't shed a tear, they don't squiggle, they don't worm, we don't have to restrain. Then others it's just a hot mess (laughing) from start to finish, so I think pain medicating is rough.

Two policies were located that addressed pediatric pain: an extremity injury protocol and a pain treatment policy for the ED patient (Appendix C). In both policies the administration of weight based analgesics specifically Tylenol and Motrin were recommended. For more obvious skeletal deformity in the pediatric patient 3 to 6 -yearsold Tylenol with Codeine elixir $5 \mathrm{ml}$ PO and in the patient 7 to 10-years of age Tylenol with Codeine elixir $10 \mathrm{ml}$ PO. Physicians and mid-levels were good about asking parents after the initial examination of the child if it was okay to administer Tylenol or Motrin for the child's pain when appropriate. While protocols were written to address the pain for a child's chief complaint of illness or injury no participants were observed to use pharmacological interventions during the implementation of needle work that involved IV insertion, or blood work.

The use of L.E.T mixture gel was the most regularly used treatment prior to suturing any individual; however no formal policy was located that supported the implementation of the practice. The use of L.E.T. gel was understood as a given treatment for a patient who presented to the emergency department with a laceration. 
Non-Pharmacological. The term non-pharmacological treatments gave pause to many participants, and further clarification of the concept was necessary. To participants, non-pharmacological treatments were viewed as comfort measures. For them, comfort measures addressed the physical or psychological needs of the patient through an appropriate and timely intervention that projected caring and empathy. The implementation of comfort measures was intermittently observed in the care of children during procedures. For participants, the goals of comfort measures were to make the experience more tolerable.

( Lizza) Because children don't understand they're immature their brains, they don't understand what we're doing or why... anything that you can do to make that a pleasurable, ah, a tolerable experience, because I don't think coming to a hospital is pleasurable for anybody, but tolerable to a child so that they're not so frightened that they know it's a safe place, that they know they can go to again if they get sick, that the staff is going to treat him nice.

(Graham) I think their comfort matters. I mean do I see a difference in their pain scale? No, but if they're comfortable they're more willing to go through the experience where as opposed to not fighting it tooth and nail from start to end. I mean you could some how, just find one connection, you know I think you're starting to get them into a comfort zone, they're not going to be overly comfortable because they are out of their area, but I think you can see them being more at ease, they're more willing to allow you into their world, into their personal space whatever that is, that arms length distance, you can get into their personal space more easily.

When speaking to the staff and participants, they prided themselves on the fact that they had stuffed bears that they could give to children; however the distribution of bears was highly dependent on who was on. Certain providers were more apt to give a stuffed bear as a comfort measure, while other providers did not give anything. Rewards were used rather infrequently, while there was an entire room filled with stuffed bears, the bears were not given out on any regular basis. Graham was asked about the teddy bears:

We have them, nobody gives them out, I mean this back room is just like filled with them. It's not easy being in an emergency room especially for a kid you don't 
understand what's going on, all these big people doing stuff and there's no payoff. I think as a kid and it's all about getting something that you enjoy even a little teddy bear or book or something it kind of lessens in their mind the whole experience, the traumatic experience of coming to an emergency room.

I keep some [bears] in a box under the Pyxis [medication distribution unit] for when the kids are crying. When asked as to when the bears are distributed, the tech replied, both sometimes we say you want the bear then you have to do this, but sometimes they're so scared it doesn't matter.(Field Notes, 04/13)

The Rule of Efficient Delivery. For participants, the rule of efficient delivery was a favored technique to decrease psychological trauma, and discomfort for the child. The idea was to perform an invasive activity with speed and efficiency to completeness. This was best illustrated by the following participant:

(Ginelle)To get it done with as quickly as possible and without causing too much stress to the child and the parent, to be quick and accurate. You want to be quick because you don't want them to be stressed for a long time and usually once it's over they are okay with it, and then of course you want to be accurate because you don't want to stick them twice. Whatever it is that you are doing, you want to do it quickly to get it over with, so that the anxiety will be reduced. So get it done, get it done right the first time, and get it done quickly so that they can get over that trauma.

The rule of efficient delivery was considered such an important aspect to managing a child's procedural pain that one participant adopted a special term for the technique and referred to it as the "gone in 60 seconds rule" a pun of the fast paced car movie with Nicholas Cage. Lizza describes the technique:

The biggest stress of a child is being held; believe it or not it's not so much the needle stick, sure it hurt's for the 10 seconds that you're going in to draw blood or put an IV in, the younger ones they stress about being held down the most. So I have a gone in 60 seconds rule for I would say 6 to almost 14 because they stress about the needles. You have everything ready, you come in, you don't say too much, well we need to take the little blood here, needles in and out, and you're gone in 60 seconds before they've had timeto think about it, to stress about it or cry about it, so I have a gone in 60 seconds rule.

Healthcare Providers' Perception of Powerlessness. Powerlessness for the healthcare provider refers to an individual's lack of control to implement an appropriate 
treatment in order to affect a better outcome for a child's pain management.

Organizational unit structural hierarchy was designed with the medical director and the ED attendings at the top of the pyramid these individuals were viewed to set the tone for the current state of how pediatric procedural pain was addressed and treated. On different occasions participants acknowledged the potential benefit of using topical agents to assist with needle procedures, but defaulted to the idea, "we just don't do that here". The same was true about the use of benzodiazepines, sedatives, or opiates. This is best illustrated by this participant:

It is good to be an attending because attendings do what they want to do, but as a mid-level (physician assistant) we practice in their zone of comfort. Here the older guys are not comfortable [giving benzodiazepine to young children prior to a painful procedure]. For some it may or may not be a moral issue, but the older guys don't want to. Attendings do what they want, we do to make the attendings happy. (Field Notes, 07/12)

Other participants shared the idea that there was only so much that could be done without receiving an order from the physician.

Umm, none. I mean to give meds. It depends on who's on. You know you can if you have a good relationship with the doc that's on, you can ask them to give something, but most of them. Most ER physicians are not good with kids, they're just not, they're not used to taking care of them. (Maura)

Well, the main thing stems from physician ordering, you know they're the one's that have to, I mean you can put a bug in their ear, but if, if they're going to give Toradol for a fractured arm, I'm going to know that's not going to cut the mustard. So it has to stem with the physician. (Lizza)

I don't know, I just think it is also an education on the doctor's part too. If they started demanding it (she looks at me and opens her eyes wider), you know, but they have a different focus. There is a different focus with them. (Kate Lyn)

\section{Summary of Treatment of Pain}

Pharmacological treatment of pediatric procedural pain was viewed by participants to be dependent on the degree of power or authority of the healthcare provider. Participants 
who were considered to be under medical supervision found lack of physician

prescription a limiting factor. Pharmacological therapy was limited to the use of topical anesthetics prior to suture application, when appropriate. Analgesics were generally offered to children who presented with some type of musculoskeletal trauma, but not always proactively before painful procedures such as radiological manipulation. The rule of efficient delivery and non-pharmacological treatments were practices more within the direct control of nurses' and technicians'.

\section{Communication Related to Comfort}

Various communication behaviors were observed in participants during the care of children. Behaviors are summarized in Appendix H. The most frequently used communication behaviors during a procedure were: reassurance, positive reinforcement, parental presence, and positioning. Reassurance was both verbal and non-verbal. Verbal reassurance refers to the verbal responses by the healthcare provider to boost or promote psychological comfort in the child or parent. Examples of responses to children included: “it's okay”, “it's okay I'm here”, “all done”, “you won't feel it” and "it's not going to hurt." Verbal reassurance was also used frequently by participants to minimize a parents concern that the child was not experiencing pain. These behaviors are best illustrated in the following case study:

Case Study: The physician sets himself up and prepares to suture a 2-year-old; he says to the young girl, "we're going to put on pirate patches". Her eyes are now covered, the child struggles against the hold of the technician and cries. The technician maintains a firm grip of the child's head to limit the movement. The sweat from the technician's hands make the gloves cling tightly to his skin. MD: Little pinch, you're the best, no move. Nothing will hurt her now. (The physician states with confidence).

The child is screaming and crying as she attempts to break free against the hold of the three people securing her.

Dad: We'll go see brother? The child continues to cry and scream. 
MD: Nothing hurts almost done.

Grandmom: Making all better. The grandmom attempts to break the constant crying and screaming.

MD: Nothing hurts anymore honey. You're doing fine honey. Her scream may become severe, she's not in pain, she's very angry.

Though the girl is screaming and crying she is able to yell out, "I want papa!!"

"Nothing hurts, nothing hurts, you're okay baby girl, such a good girl", says her grandmom. The child continues to cry and scream, then she starts coughing and choking on her saliva, but no one moves they let her cough and cough.

MD: Nothing hurts, you're okay.

Dad: Talk to daddy.

Child: NO! NO! I want mommy (she coughs and gags). The MD was attempting to open a suture package but dropped the package on the ground, the physician kicked the package back to the red garbage can and yelled out for the RN to come into the room and get for him 6.0 P-2 suture material. The child continues to fight, cry, and scream.

MD: You don't hurt so everything is fine.

Grandmother: Close your eyes baby, I'm right here, I'm not leaving.

The child continues to scream and cry. (Field Notes, 06/11)

The child's constant verbal resistance, screams, cries, and attempts to break free

visibly disturbed the family to the point that the mother had to leave the room. In this case study, the use of verbal reassurance was used to address the family's concerns for the child's pain.

Non-verbal reassurance refers to the use of touch and spatial positioning (i.e., position to eye level of the child) by the provider as a way to build trust and console the child. Examples of non-verbal reassurance included kneeling on the ground to be eye level, giving hugs, high fives, wiping the forehead of a child, the provider rubbing or patting a child's head, rubbing a child's chest, arm or shoulder during a procedure.

Case Study: A 33-month-old with a laceration to the nose. The location of the injury does not allow for the administration of a topical anesthetic. The child must be restrained and injected with Lidocaine to the injured area. The nurse secures the child's head and the mother secures the child's legs. At the end of the procedure while the child is being released, the nurse rubs his hands through the boys hair as he backs off from securing him. (Field Notes, 04/25) 
Positive reinforcement refers to the act of praise or reward to increase the rate of expected good behavior. Participants statements included: “you're a big girl”, "you're doing a great job", "big boy", "you're a brave boy", "you're so good”, "what a good patient", and "good job very brave." The use of positive reinforcement is illustrated in many of the examples provided in this study.

Parental presence was a consistent comfort measure implemented by participants. Parental presence refers to the parent present with their children during a procedure. In only one case, a mother had to step out of the room and was replaced by another family member to comfort the child. Parental presence was a general expectation of both the participants and the family members it was never vocalized as an intervention, but as an understood given.

Positioning as a non-technical comfort measure was used most frequently during the initial examination of a child and after a procedure. Positioning refers to placing the child in a therapeutic position that facilitates the child's comfort. The child sitting in a parent's lap or held in a parent's arm was the positioning technique of choice in a majority of cases. In contrast, the use of restraint was conceptualized to refer to the physical hold and stabilization of a child using people, equipment (i.e., drawer sheet, pillow case) or both.

Participant behaviors prior to a procedure involved education specifically procedural preparatory information, sensory preparatory information, and child directed speech. Procedural preparatory information was a common non-technical comfort measure directed to parents. Procedural preparatory information involved the depiction of the steps of the procedure, without descriptions of sensations. The amount of procedural preparatory information provided varied among the physician, mid-level, or nurse 
dependent on who had first contact with the child-parent dyad and the type of procedure to be performed. Participants always emphasized that approach of a child was age appropriate.

I explain the procedure to the child I let them look at the equipment I am going to use, if it is age appropriate of course, I try distracting them and it works...you can still explain something to a 2-year-old in a way that something gets across, they can understand. You know how you handle them, how gentle you are with them, your tone of voice as opposed to somebody who is being rough and says, oh we're just going to draw blood, if you're soothing and you're touching them and you're gentle with them, I think that plays a role. Cause a lot of people think, oh it's just a kid you don't need to explain things to them, but they're people they need to know what's going on, when you explain things to them even though they're still scared they still have a little bit more of an understanding, they might feel a little bit more comfortable with the person providing the care and doing the procedures as opposed to going in and saying we're just doing an IV that's it, done. (Lain)

For the younger patients, procedural preparatory information was always geared toward the parent. The participant's lack of ability to educate a child to what needed to be done was a source of frustration.

(Graham) I don't like to hurt somebody, if I'm doing something that's borderline uncomfortable I like to be able to explain the reasoning behind [it], I mean I have to do it often you can't explain why, I don't like that forceful medicine, where its pretty much like this is going to happen deal with it and I have too...Nobody wants to be treated like that and like I said if you're going to do something that it is going to be borderline or extremely uncomfortable and with kids you can't they may act like they understand I mean like 5, 6, 7you could talk to them a little bit, younger than that give it up you speak to the parents.

Along with procedural preparatory information participants elicited parents "buy in" or cooperation on how the procedure was to take place important points addressed the need to getting the procedure done, the child's "normal response" of fighting, and completing the procedure in a timely manner. This participant's response illustrated the normal practice of eliciting parents' cooperation in the ED:

(Ginelle) To hold them and to comfort them and that you're going to do what you need to do and if they can hold them well it will be done quicker. The more they 
participate and the more they help in keeping that child still the quicker we can get everything done.

A 23-month-old girl came into the emergency department with a foreign body in her nasal passage way. The mid-level (physician assistant) went into the room and spoke with the father, "I think that we might have to papoose her. I'm going to get it out with suction she won't like to have it done, kids this age don't like it." (Field Notes, 04/13)

For some participants direct interaction with a child required the use of sensory preparatory information. Sensory preparatory information provides a description of sensations (noises, smells, physical sensations) that a child will experience during various points in the procedure. The following case study illustrates the use of sensory preparatory information during a simple procedure taking the child's temperature and blood pressure to the more involved procedure of starting an IV line with blood work:

The nurse sees the patient walk into the ED room. The child is 4 years of age brought in for evaluation of vomiting and diarrhea.

Child: I want mommy!

Nurse: Mommy's here, she'll be here all night.

Mom: I'm right here.

The child climbs up on the stretcher. The nurse walks over to the monitor attached to The wall and says, "the cuff will make squeezy, squeezy, on your arm."

Child: I'm being a goot girl.

Nurse: It makes lots of noise, it will make squeezy, squeezy.

Child: Thank you.

Nurse: Have you seen E.T.?

Mom: No, she's afraid of the alien.

Nurse: Give me your finger; it's not going to hurt... Let me see if I can find another thermometer. Let's see if this one will work better. Is it working? Yup, close your mouth, under your tongue, sit still don't move it, it's like sucking on a straw.

Later on the nurse walks into the room gives Zofran and places the medication under the child's tongue. "Taste like gum, right? Taste like gum?" The nurse takes mom to the outside of the room and talks about concerns of fluid loss and need for replenishment. The mother agrees to the fluids.

Nurse: Let me get some holding help and then I'll come in.

The nurse goes to collect more supplies and obtains an arm board. The nurse sees the mother walking out, and says, "I'm going in mom, just so you know." The mom changes directions and walks back into the room.

Nurse: I'm bringing up the bed to me (she pumps the bed to make it higher). Squeezy, Squeezy (she places the tourniquet on the child's left arm). The tech holds 
the arm in place as per the nurse's direction. You're so brave, you're so good. The nurse turns on the pump and says, "ringy, dingy as the pump bell goes off."(Field Notes, 05/17)

Other comfort measures used by participants consisted of child directed speech. Child directed speech refers to speech patterns used when communicating with young children such as simplified vocabulary, melodic pitch, or change of intonation to high pitch. Common statements consisted of "did you smile for pictures", when referring to xrays, "can I clean your boo boo", and "let me cut your whiskers" when referring to sutures. According to participants, the use of child directed speech is guided by the child's developmental level. This is illustrated by Lizza:

Age appropriate is first, you got to know your age appropriateness, you can pretty much assess their level of maturity if they're clinging on with mom or not, if they're the minute you come in and they're (she simulates crying, whining). You know, they immediately withdraw you just looking at them sometimes. Some of them are just too sick and that has a lot to do with it too. Depends on how sick they are, if they have obvious boo-boo and they're not crying, awe we got to fix your boo-boo, we're gonna put some medicine on so the boo-boo doesn't hurt. Talk age appropriate I wouldn't say you got a boo-boo to a 14-year-old (laughing) it all depends on how they act when you come into the room to greet them.

Deviance from Communication Related to Comfort. While participants used a variety of non-technical comfort measures to care for children during procedures some behaviors violated the norms of observed practice. Deviance from comfort behaviors were identified as criticism, condescension, and dismissive remarks. The following observations illustrate some of these points:

The triage nurse comes in; "I need to use room one to draw blood work on a little girl." The patient is walked into the room. The triage nurse rolls in a tray and the girl starts to scream. I see the tech walk in and the girl hides in the corner of the room and pulls away from the mother's hand. The nurse kicks the tech and I out of the room and pulls the curtain. From the outside of the curtain the dialogue is clear. The nurse in a loud voice attempts to talk to the girl "this is what I am going to do; you're going to feel my two fingers and a little pinch, that's it." The girl continues to cry. 
(Later) Some holding help is found. The tech says to the girl, "I'm not going to let them hurt you, you're a big girl, you're a big girl." The triage nurse directs the tech to secure the child's arm; the tech went from the left side of the stretcher to the right side to hold the arm and gently rubs the girl's left shoulder. The mother is rubbing the child's chest and squeezing her face to turn her face away from the procedure. The triage nurse says, "You know I have a 7-year-old boy and I would like to get home to him, there's a lot of people waiting out there for me, but I'm in here, I can't do this all night." (Field Notes, 04/13)

A 7-year-old is brought into the prompt care area.

Nurse: "Hi, how did you fall?" The father explains that the boy was at school and he went to the bathroom and when he came out he ran and couldn't stop and banged his head. The nurse goes to prepare the medicine. He walks back to the room, "Okay young man this will numb the area. Touch it it's cold."

Child: What does numb mean?

Nurse: You won't feel it, then I wrap your head. Okay let us leave it on for 20 minutes then the doc will fix it.

Child: How will he fix it?

Nurse: I won't ruin the surprise.

(During the sutures) The child is lying on a partially upright stretcher the nurse is standing at the head of the stretcher attempting to secure the child's head. The physician is standing on the left and the father on the right. "Hold still, don't move", the boy goes to pull himself away from the source of pain he retracts to the side near his father and pulls his body down to curl into a ball lower on the stretcher. The boy is crying and screaming the sounds are transmitted throughout the prompt care and zone three. The physician injects the Lidocaine, the boy loudly screams. "Now you know what stinging feels like," says the nurse, the boy continues to cry. (Field Notes, 05/01)

The little girl started to cry, the physician walked over to the right side of the stretcher took his index finger and gently swiped the bridge of the child's nose, "Sadie, what did we talk about?" The mother stood at the young girl's feet looking down at her with heartache watching her child cry. The doctor said, "she's okay" the mother leaned over and said, "I can't do that." She picked her daughter up and started to rock her back and forth trying to calm her down, "I can't listen to her cry like that". The physician looked at her and said in a very frank manner, "We're going to hold her down, she is going to scream like we're killing her, if it's something you can not handle maybe better if you leave the room, I'm not telling you what to do, but it may be something you have to do. The mother laid the child back onto the stretcher, the physician directed the father to secure the legs and the mother to be within eye sight of the child. (Field Notes, 06/11) 


\section{Summary of Communication related to Comfort}

Various communication behaviors were used by participants' pre-procedural, procedural, and post procedural. Pre procedural communication behaviors emphasized procedural preparatory information, sensory preparatory information, and child directed speech. Communication behaviors were normally tailored to fit the developmental needs of the child and family these included: verbal reassurance, non-verbal reassurance, positive reinforcement, parental presence, and positioning. Some participants displayed deviation from standard communication related to comfort that included criticism, condescension, and dismissive remarks.

\section{Fragmentation of care in the ED}

Fragmentation refers to the breakdown of care from one compartment of the emergency department to the next and the handing over of patients to different providers. Fragmentation decreased the ability for the child and family to develop a meaningful rapport with providers. For the purposes of filtering and funneling patients in the ED, the delivery of care was compartmentalized into three areas triage, prompt care, and the main ED. In each of those areas, depending on the activities of the department it was possible for a patient to be approached by a different nurse, and/or technician for the completion of certain tasks such as vital signs, blood draws, IV starts, and splinting. The comments below describe some of the process that occurs within the triage waiting area.

When patients come in the first person that assess them, or does a visual assessment is the nurse (he points to the chair and laptop station in front of the triage booth). At this point, the nurse gets the pulse ox, respirations, pulse, general complaint and maybe temperature. Then if they think that patient needs to be seen I will see them. In this room there will be either a physician assistant or a nurse practitioner, and depending on what we find on examination then we will send them into the room across the way, the triage treatment room, to have bloods, IV's, and medications." (Field Notes, 04/11) 
Two of the participants vocalized dissatisfaction with the care of patients in the triage area:

Sometimes, it's just the tech drawing the blood while the nurse is typing away, you know they're putting a PA out there to try to order some of these tests, they're actually using the waiting room as another ER area is what they're doing. I'm not too happy about that business patients are getting stuck twice. Yesterday I had a girl who [had an] aspirin overdose they did levels out front, brought her in the back, she needed an IV to flush it out and got stuck a second time, it happens a lot. (Lizza)

I have been there for five years and there's always been a mid-level in the ER and the triage area. I'm not there during the day so it's hard for me; I know they're there when I come in at 7 p.m. and they leave at 9 p.m.. I understand what you're saying as far as the care is fragmented because they're [patients] seen by this provider, this one's ordering this, they go to CT scan and still have to be seen in the back. I think that in order to address a fact that you come in you're seen within 15 minutes by a nurse and then 30 minutes by a mid-level the 15/30 rule which is being enforced now. It's kind of hard to do that, to be honest with you I don't feel comfortable being out in triage when that is when there's too many people in the process; something is going to be missed. I feel uneasy being in that first primary triage role. (Lain)

Refer to Appendix E for Fellowship Hospital's emergency department triage and assessment policy. Most children were quick triaged by the first nurse, sent to radiology when needed, sent to the prompt care center where ideally the patient was seen right away, however the timeliness of patients rotating through the department required the providers to quickly move in and out of rooms and delegate tasks when appropriate to a nurse or technician whoever happened to be available. The streamlining of patients through the department limited participants' interactions with children and their families. During high volume times full staffing in the prompt care center consisted of one physician, one mid-level, two nurses, and one technician. In the ED, the staffing assignment varied due to the shortage of staff nurses, personnel sick days, and uncovered vacation days. During days of low staffing and high volume the quality and delivery of care to patients declined. 
I saw that there was a 4-year-old on the ED board with vomiting and dehydration to be placed in one of the ED rooms- time 18:18. When I went to the main, no patient was in the ED room assigned. After the change of shift report, the nurse who was supposed to receive the little girl started to take orders on a new admission patient and then was transcribing orders. The nurse called report to the floor on two patients with assigned hospital rooms. The documentation of the little girl from triage is scarce with only a pulse; there is no documentation of pain or other vital signs. The nurse sees me and asks, "What ya doing taking notes?"

Researcher: You're getting a kid.

Nurse: I don't see anyone in there.

The 4-year-old has been waiting for one hour and twenty-four minutes. The nurse checked the board for the chart of the girl.

Nurse: NO BP! NO TEMP! NO vital signs, no nothing. The charge nurse will bring her in.

The charge nurse from the day and night shift are working on the schedule.

Nurse: You got a patient going into 3?

Charge Nurse: Yeah, I don't know where she is.

The charge nurse picked up the phone and called out to the triage registration area, "hi we clicked the 4-year-old a while ago, do you have her out there (19:50)?"

(Field Note, 05/17)

In the above case, the child was left in the waiting room even though an empty ED

room had been assigned to the patient. The busyness of the department and the

expectation that someone else was responsible for placing the patient in the room delayed treatment of care between triage and the main ED to the 4-year-old girl. The basic

assessment in triage was incomplete and it was not until one hour and twenty minutes later that the child's care was started.

\section{Summary of Fragmentation of Care in the ED}

The sort, funneling, and streamlining of patients through the waiting room, triage, radiology, and prompt care or the main ED contributed to the breakdown of quality of care for the pediatric patient. The focus on turning patients over in a timely fashion with the emphasis of ordering tests in triage and having them completed prior to medical examination neglected to address the pain management needs of children, especially those with musculoskeletal injuries. 


\section{Healthcare Provider Attitudes}

Healthcare provider attitude refers to either a positive or negative evaluation related

to the participant's beliefs about pediatric pain.

Uneasiness of Staff. Uneasiness of staff refers to a generalized feeling of discomfort

by the provider due to the lack of practice of taking care of and performing procedures on

young children. This was illustrated best by the remarks of these two informants:

Lot of the nurses don't like to get peds [pediatrics] and the charge nurse will actually ask, is it okay that I give you this sick kid, cause some nurses don't like to have the peds. I guess they get to have a choice, but it's funny I said, why'd you ask me that and she said because a lot of the nurses, I thought of you too that day, she says a lot of the nurses don't like to get the pediatric cases...You're not going to find experienced peds nurses out there because they don't exist, all those nurses have been there for years, they're used to their adults, they don't have that many peds. (Lizza)

I'll be honest with you a lot of people are uneasy about taking care of kids because they don't. The comfort level isn't there... a lot of them say, well I don't want to stick them, I don't want to induce more pain, if it's an IV stick, can you do it for me? I mean I'm asked a lot, I'm comfortable with kids. I don't mind doing an IV and helping out. You have to be comfortable in order for you to do it...in order for you to feel comfortable with a child you have to do it on a regular basis and not every body is comfortable working with children...I mean if you deal with something on a day to day basis you just become more proficient and more knowledgeable as opposed to somebody who doesn't work with kids on a regular basis... It's a cultural thing if you don't deal with it on a day to day basis. I mean if you asked an ER nurse to deal with a person with chest pain they can do it right away as opposed to dealing with a child who comes in with another type of injury. It's the fact that you don't do it on a daily basis that plays a factor in it. I think experience and dealing with kids is important, you have to feel comfortable.(Lain)

During an informal conversation, the comments made by Lizza were supported by

this participant who was very vocal, "I don't do kids":

Hi, I am the float also known as the whore, (she covers one side of her mouth to quietly whisper her moniker) basically I do the things that no one else wants to do. You probably won't see me with kids, I don't do them. I just don't. It's not that I don't like them, it's just that I don't do them. The other night Kasey was here and I called her for the 2 kids we did have. I don't do kids (Field Notes, 04/13). 
The biggest barrier for this participant was identified as lack of knowledge:

Lack of knowledge, end of conversation. I didn't know what I was doing. I was always nervous around kids, overdoing them, overdosing them, because their scales and dosages are so different.(Ginelle)

Parents. For some participants, the parents of a child contributed to the uneasiness of caring for children. Behaviors identified by providers consisted of parents who hovered over healthcare providers and asked incessant questions related to the care of the child. Display of those types of parental behaviors forced providers to spend less time with children. This is best illustrated during an informal conversation with a nurse participant:

I don't like other people's kids. I don't like when the parents are (and she places her fingers like a claw over something) hovering. Just let me do what I need to do. (Field Notes, 05/11)

The parents make it real difficult, I mean they're more protective, they're more overbearing of wanting to know why is this being done, what happens after this is done, which are good questions, I mean they're good questions. Sometimes for me as a tech it kind of puts me on the edge, it shies me back of my approach. (Graham)

Passing the Buck. The following informant illustrated the dynamics of "passing the buck" a description of what unfolds between providers when invasive procedures are needed on children and the provider is not comfortable:

I don't like seeing 'passing the buck', so with my colleagues that don't pass the buck I mean, if it's yours own it, good, bad or indifferent, but don't pass something that in your mind is prone to failure or is a task that you really don't feel like doing. Passing the buck is taking one of your duties that you have plenty of time for or if you don't have time it's still your duty it was given to you by somebody, and you don't feel like doing it because I'm afraid of failure, so I passed that task that, that I believe I'm probably going to fail at it, you have that feeling obviously, I don't know, so you pass it off, that's passing the buck, taking one of your rightly owned duties, and handing it to somebody either on your level or below your level that is still capable of doing the task. Almost like negative delegating. (Graham) 
With regard to invasive procedures in the triage area many of the participants highlighted that drawing blood from children was reserved for being done in the main emergency room:

We don't do a lot of blood work on the younger kids; I think it takes so many people to hold them down it might be better to manage them in the back. Once they get their blood work in here they go out there [the waiting room] and no one wants to listen to a crying kid for an hour. (Field Notes, 04/13)

Many participants agreed that children were extremely fearful about getting needle procedures, but did not believe that pre-medication prior to a simple needle procedure (i.e., drawing blood, intravenous catheterization) was necessary. This is best illustrated by the remarks of this participant:

It's never been considered to that degree of pre-medication prior to because it's just so quick. I don't think that is a hospital policy to pre-medicate for an IV because you go to a LabCorp or you go to a doctor's office to get a vaccine, you don't get premedicated. I don't think, I don't consider the level of pain that bad, I mean pain is pain, when you see an IV and let's say suturing it's two different ends on the same thing. One is really involved and continuously suturing you're doing it multiple times. An IV stick is just a one time thing, that's it. (Lain)

Consequences. Many participants described the overall experience of getting stuck

with a needle and held down as very traumatic for a child:

It's a traumatic thing; it's a traumatic thing for a child to be stuck with a needle, but if you get it done quickly, efficiently, you're done, you're out, they're with their mother and father. (Maura)

When asked about concerns for potential long term negative consequences participants varied in their responses. Some participants did not believe there were any long term negative consequences related to the little pokes from blood draws, IV's, or sutures.

(Ginelle) No, no absolutely not (She says this with confidence and certainty). Because they are resilient and they forget about everything the next minute. Again, just get everything done as quickly as possible because you know the child will be 
calm when it's over. Once it's over they'll be okay, you know doing everything and getting to that spot that is giving them anxiety and fear, but once it's over they're okay because they bounce back.

(Kate Lyn) Not if you're honest (her voice increases in pitch and tone), not if you're honest, if you're talking to a 2-year-old they're normally relatively sick that they're not going to remember. If you approach it in the right way, you have a good outcome.

After suturing a child, one physician participant spoke about the amnestic effect in children three years and younger. This physician believed that younger children were unaffected by traumatic events because they did not remember.

You know that up to the age of 3 the kids are amnestic to the event [sutures]. Do you know how we know? We see them in the office and they're happy to see you. (Field Note, 06/11)

Some informants did believe there may be long term negative consequences related

to the little pokes from blood draws, IV's, or sutures.

That's Pavlov's conditioning, you know bring your kid to the pediatrician and he gets a shot every time he goes he's not gonna want to go to that pediatrician. If you take your dog to the vet and he gets blood work and shots every time he walks through the door my dog starts shaking, and kids when they've had blood work or been to the hospital especially children with cancer or children with major illnesses that require a lot of needle sticks, already have that anxiety getting in the car cause they know its coming. No doubt about it. (Lizza)

I guess it could be a traumatic experience for a kid; I mean and could scar them for life. If done incorrectly, yeah, I'm a big believer in that. They grow up and not want to come to a hospital when they actually need to cause that stuff sticks in your mind. What, a kid most learns by age 5. So that means, their mind is very impressionable at that age. It lets me believe that that age group of younger are going to remember things that happened during that time of their life further down into their adolescence and adult life, and I don't want to be responsible for scarring somebody emotionally and mentally when they're a kid doing something, and when they need life or death help of a heart attack or stroke or something in their middle-aged life that the one thing sticks in their mind that I did that's going to prevent them to come to call 911 or getting into their car and driving to an emergency room. I try to give the caring touch so that everything that happens to them here today isn't bad, so you can take something positive away from it. (Graham) 
At first glance it would appear that addressing pediatric procedural pain was very sporadic. Individual characteristics became a predisposing factor in the treatment of children; some providers appeared to be more attuned to the pediatric patient needs. Whether a provider was a parent or non-parent, and taking into consideration years in practice yielded very little in the predictability of how children were treated. A better indicator was a participant's desire to work with children. Providers who expressed wanting to work with children were more apt to go out of their way to address the pediatric patient needs. There was a visible difference in those participants who went through the motions, and those who individualized the manner in which care was delivered. Participants who shared this attitude were more likely to go out of their way to ensure that children were comforted during their stay.

I don't want them to leave the hospital thinking it's a horrible place. I want them to leave the hospital and know that that's a place they can come to where the people are going to treat them well. (Lizza)

The following excerpt exemplifies how a participant individualized the manner in which care was delivered.

A 3-year-old male has a cut to back of his head. The pain score is zero. History is the boy tripped on a blanket and fell on back of head. As per mom, no loss of consciousness. The patient was walked back from triage.

The mid-level (nurse practitioner) walks into the room and examines the boy's wound while he is on his mother's lap, "not bleeding we just tie the hair together". The mother says, "really?"

Mid-level: No, his hairs not long enough to do that even if I wanted too. Okay, this is how it goes we could $S-T-A-P-L-E-S$ (spells it out). I could try gel but generally it doesn't work for this area (scalp occipital region) that well. I could stick with $N-E-E-$ $D-L-E$. I leave to mom how to handle.

The mother asks questions.

Mid-level: The Lidocaine provides extra relief 10-15 minutes after the procedure if $S-T-A-P-L-E$. It would be two procedures versus one. If I do nothing, it will B-L-E-ED. It's not like it would be dangerous to leave it.

The mid-level shows the mom the staple remover kit.

Boy: Can I go home? 
Mid-level: How's it feel?

Mom: Hurt a little.

They talk about the Lidocaine, "it's pain no matter what, pain is pain," says the midlevel.

Mom: We'll do the one, I'm afraid he won't sit for the other.

Mid-level: Oh he'll sit, we'll make him sit, that's not the problem.

The mother talks with the little boy about fixing his boo, boo, and stop for ice cream on the way home.

Mid-level: You'll deserve it.

The mother lies on the stretcher and holds the boy, she says, "lets play I spy." They look at the picture on the opposite wall of where the mid-level is setting up.

Mid-level: Can you look at the picture?

Mom: See trees and the water?

Boy: No, I don't.

The mid-level leans in to give the needle "just a little pinch" the boy starts to cry. Mom: It's okay. You're so brave. You're so brave.

Mid-level: Done, want to see what we did?

The mid-level brings over one of the staples and shows the mother and little boy. "You're like Robo Cop. Tell daddy you're the bravest boy. You want to show them (dad and brother). I'll put in a special tube.

Boy: All done. Mom we going home?

Mid-level: Let me put in tube for him. You tell them you're the biggest bravest boy. Nurse documentation: Pain level 2 hurt little bit using the FACES pain scale. (Field Notes, 05/07)

When participants were asked about using pain scales before, during, and after

procedures the question elicited similar responses, one participant stated (Maura), "you

consider it [pain of the procedure], but it doesn't come into play."

Well you know what they're going through before, and you know that they're going to have some kind of pain when you're doing it and then after you make sure that they're okay. You know that they're anxious and that they're just going to be screaming. Do you really have time? (Her voice goes up). You got to get that IV started you gotta focus on finding the site getting the proper equipment and getting it done. (Ginelle)

If participants documented the child's response to the procedure, the most common statement written would be patient tolerated procedure well. This broad statement lacked in the specifics as to the amount of pain experienced by the child, if therapeutic measures were offered to alleviate pain, and if restraints were used. Documentation for the 
procedure was more focused on the technical aspects such as the site, size of the catheter, amount of times the child was stuck with a needle, blood work drawn, labeling blood work in the presence of the patient, and items sent to the lab.

\section{Summary of Healthcare Provider Attitudes}

Participants offered a variety of responses to their beliefs about pain and the potential consequences related to the under management of pain for the pediatric patient. Parental presence was viewed by some as a deterrent to optimally delivering quality care. An underlying concern described by most informants was the general uneasiness of ED staff for providing care to pediatric patients. Uneasiness contributed to practices described by some participants as passing the buck.

\section{Environmental Issues and Policies}

Environmental issues refers to unit specific priorities that influenced participants behaviors and actions to the overall care of pediatric patients during procedures in the ED. Environmental issues consisted of keeping the flow, and time management preparing for uncertainty.

Keeping the Flow. The idea of moving things along or keeping the flow was formulated on the unrestricted intake and output of patients, the fast-paced nature of the department and the need to turn patients over in an efficient manner. Keeping the flow was a recurrent phenomena noted to be an important aspect to the functions of the ED. This was illustrated best in the following examples:

We are busy and we are at full staff. We are a very busy emergency department. We have to move patients it is clogging up the system. We're holding six patients (she lifts her arm in the direction of the waiting room) the waiting room is not so good. I wish we had a sign of what to expect. The people need to see their own doctors cause we will take other patients ahead of them and they can wait a long, long time. We're down a doc [the prompt care physician went home], zone three closes, is only open 
from 8 a to 11p, then we are down two docs and they're six charts behind. (Field Notes, 04/11)

It's a tough area working in the ER because you have to do what you have to do in order to address whatever issues arise and it's hard, it's hard, I don't know how it can be addressed other than to comfort the person, but you also have to look at your timing as far as you can't keep everybody there for like an hour or two trying to get an IV done...especially if it's abdominal pain. You have to get the blood work done, send them to CT scan, see if it's appendicitis, time is of the essence. So it's hard, you don't want to medicate them prior to that because some of the docs don't like medicating prior to them examining them [the child] with any narcotics because it might mask whatever pain they have as far as their assessments for the surgeon when they come in, but we do medicate patients. We try to address patient's pain as much as we can. (Lain)

The consequences of not keeping the flow are best summed up by this informant:

(Graham) It's crucial, you lose the flow and the lobby gets backed up, and CT scan gets backed up and ultrasound, I mean all the different departments that, that we work along side of and if you don't keep the flow moving and communicate it just gets bad, it gets bad, it makes the shift horrible. I could look at the situation, and say it could affect that we're trying to rush people along so much that you lose the kind, caring, touch.

Another dimension to keeping the flow was the importance of meeting recommended door to nurse times 15 minutes, door to physician times 30 minutes, emergency severity index triage acuity level 1 through 5, and the use of a built in computer monitoring time table that measured the length of stay for patients and held healthcare providers accountable to see and discharge patients in a timely manner.

On the main computer screen there was an item referred to as RN clocks. The clock turns from green to yellow to red like a traffic light. Green indicates greater than $26 \%$ RN time remaining, yellow indicates less than $25 \%$ RN time remaining and red less than $10 \%$ $\mathrm{RN}$ time remaining. The color codes remind the nurses to document hourly, and for those participants located in the prompt care area of the ED it reminds them that the ideal is to move the patient within a 90 minute time frame to get the patients in and out. 
Fellowship Hospital's internet webpage for patient information about the ED

reinforces the importance of keeping the flow through tracking times and sorting patients:

Ready to deliver prompt, personalized care 24 hours a day, 365 days a year. The emergency department is staffed around-the-clock with specially trained emergency physicians, nurse practitioners, physician assistants, and emergency certified nurses. Staff is supported by an advanced patient tracking and documentation system that tracks your current care...helping our team make sure your visit is as efficient as possible. Everyone who enters the emergency department goes through the triage (sorting) process, the severity of our patient's condition, how severely you are sick or injured. Arriving by ambulance does not lead to being treated more quickly. The only thing that determines the order in which you are seen is [the sorting process].(Hospital Document)

Triage was the intake area for the walk-ins, in a majority of cases these were individuals who presented to the department by walking through the front door versus individuals who arrived by ambulance; however ambulance patients who were assessed to be of low priority and were able to wait to be seen were sent to the triage area. Triage served as a systematic sort and funnel unit that utilized and was guided by the emergency severity index (ESI). The ESI is a five level triage algorithm that stratifies patients on the basis of acuity and resource needed into five groups with ESI 1 designated as the most emergent and ESI 5 designated as the least urgent. Once a patient is registered into the computer system an ESI level is assigned and color coded on the computer screen. The color coordination of the ESI levels from level 1 to level 5 include red, yellow, green, blue, and purple.

The regularly high volumes of patients seen at Fellowship's ED exacted the redesign of how patients were processed from inception at triage to discharge or admission. Two mechanisms of patient processing were updated the triage and assessment, and guidelines for an ED intermediate care unit. The excessively high volumes were evident from the stretchers filled with patients that lined the hallways. The use of hallway beds 
were to be used for patients as a temporary placement until an official room in the ED opened up; however the use of hallway beds were something of the norm because the stretchers in the hallway were labeled with laminated signs with the hallway and room number that the stretcher was located in front of so that staff knew where to find patients. An influx of patients especially on Monday's, when wait times could be up to 5 hours or more, extra green folding chairs were placed in the waiting area. During the observation period of the department revisions were conducted to increase the fluidity of patients brought in and discharge from the ED. In April the ED medical director released an updated policy for mid-levels in the triage area.

Purpose: To facilitate medical screening examination and evaluation of patients in the triage/waiting area.

Scope: Physician assistants, Nurse practitioners, Physicians.

Procedure:

1. Following triage procedure, patient will be assigned an ESI level 1-5.

2. Whenever possible, patients will be brought directly back into the main ED area or prompt care area based on level of acuity.

3. Practitioner in triage area will prioritize patients based on acuity, and facilitate a medical screening examination. This examination will involve a physical

examination with documentation of that examination and assessment in the medical record.

4. Following medical screening examination, treatment will be initiated as well as the ordering appropriate diagnostic studies by the practitioner.

5. Patients in the waiting area will be re-assessed in a prompt fashion.

6. The collaborating physician will be directly involved in the patient care of all patients ESI level 3 and higher acuity.

7. The collaborating physician will be advised of all patients in the waiting area with a work-up in progress in a timely fashion.

8. Patients may be discharged from the waiting room area by the practitioner after consultation with the collaborating physician.

9. Patient will be brought immediately into main ED if any change in condition or unstable VS.

The ED at Fellowship Hospital utilizes the emergency severity index (ESI) where

ESI 1 represents the most critical patient and ESI 5 represents the least urgent patient.

Patients are then sorted with priority given to the most critical patients. While the ESI 
systems helps to sort and detect patients in need of direct attention and resource utilization patients designated at lower levels were left to wait for longer periods of time. To address the needs of the less urgent patients, specifically designated levels 4 and 5 the prompt care area, a sub-unit it the main emergency department, was open 16 hours of the day. The prompt care area provided immediate care for non-life threatening illnesses and injuries that did not require extensive diagnostic testing. In addition an ED intermediate care (IMC) unit policy was developed, IMC patients were situated in the prompt care area.

The objective is to treat ESI level 3 patients who meet the IMC criteria. Patients with ESI 4 and 5 may also be treated in the IMC area. The following are complaints/diagnosis for treatment of the IMC. Please note this is not all inclusive list of complaints/diagnosis.

a. asthma

b. flank pain/renal colic

c. head injury

d. leg swelling (unilateral)

e. pediatric fever

f. influenza symptoms

g. urinary tract symptoms

h. acute urinary retention

i. catheter malfunction

j. abdominal pain without fever and vomiting must be less than 45 years of age.

The constant influx of patients, the need to turn patients over in a timely fashion, and the accommodation of new department policies led to a lot of visible frustration with the staff. This occurred especially during times of high volume. The following observations illustrate this point:

The triage area was backed up and the mid-level was asked by the medical director to stay longer to assist with the overflow. The patients in the triage area approached the mid-level to find out how much longer the wait would be, in an attempt to seek answers and move things along the mid-level walks to the main ED and speaks with the charge nurse. The charge nurse raised her voice in a frustrated and angry tone, "I have two medics coming in and I am 5 hours behind, prompt care has nothing, you have two nurses." The mid-level responded in a calm voice with hands raised as 
if to signal her to lower her tone, "I ask, I ask." The charge nurse turned and retorted, "everybody can just kiss" and with that she turned around and slapped her bottom. The charge nurse walked over to prompt care and assessed the availability of rooms:

Charge: There are two patients waiting for prompt care.

Nurse: I only have one room.

Charge: You've got four and chairs open.

Nurse: I don't have a charge nurse to help, I am a one person show, I'm trying to keep my head above water. I have no time to bring them in.

Charge: Where's your tech?

She looks around the area and sees the tech, "will you bring those two prompt cares waiting, thank you." Shortly after the charge nurse walks away the mid-level (physician assistant) says to the tech, "don't bring anyone else in I'm three behind." (Field Notes, 04/11)

Time Management Preparing for Uncertainty. Time management preparing for uncertainty refers to participants' constant awareness to prioritize and provide care efficiently with the anticipation that a level one acuity patient could present themselves at any moment. Emergency nurses' structured their delivery of nursing care motivated by the idea that at any moment a true "emergency" such as a full code or cardiopulmonary arrest or severe trauma would come through the department and exact the majority of their resources. This was illustrated by the following participants:

We all know that when you work in the ER your times have to be managed very well that's the one thing about working in the ER, you have to know how to do what you gotta do, so you can get on to do the next thing because it can always be bigger and worse. So definitely, time management is a big factor to being an ER nurse, you've got to know what ya got. You never know what you're going to get next. (Ginelle)

You know what; you have to be able to go 110 miles one way then 110 miles the other way, it's just something you have to do. You have to prioritize and it all depends where they [the patients] come in that priority. I mean it's a simple cold, you'll eyeball'em listen to them, there's something more important like an adult code coming in your going to that code. So it's all part [of it], you have to learn to prioritize (Kate Lyn).

The following observation was taken while in the triage waiting room:

I noticed that from the parking area there was a Caucasian woman running toward the entrance, and a Caucasian man following her from behind carrying a small child 
that appeared to lay limp in his arms like a rag doll. The woman ran directly to the triage nurse sitting behind the laptop, the nurse listened to the complaint, and automatically directed the couple to the double emergency department doors, the security guard scanned the couple in, the woman yelled something to the triage nurse, and the nurse replied shaking her head from side to side, "I'm not going to argue". The double doors opened and the family disappeared. (Field Notes, 03/28)

The lack of time management skills was expressed to be a detrimental attribute by one of the participants:

Well some nurses know how to prioritize in an ER and some don't, if I had an acute MI (myocardial infarction) I needed to get a monitor on them, line, oxygen, and an EKG (electrocardiogram) before you even walk out of that room, a hip I know I'm going to need a type and screen and a blood panel, you can see the rotation, a kidney stone they're flopping like a fish, they're flopping like a fish. You've got to prioritize, you know, people don't know how to do that, oh I gotta go do this first, this first then I'll give the pain medicine. You gotta know your patients; you gotta know what's in your spots (different rooms), you gotta know that that child came in crying and has probably broken her arm, my priority on that child would have been pain medicine before she even left the unit to go for anything. (Lizza)

Many of the factors discussed above exerted more pressure on those participants in the prompt care area especially for the nursing staff because they strived to meet the 90 minute input and output times. The nurses in the prompt care areas were responsible for complete documentation including, at times, the triaging of the patients whereas the nurses in the main emergency department had the luxury of receiving patients with triage notes written, and were responsible for the five patients they were assigned at any one time.

Well the turn over in prompt care is so quick. The nurse there has to document everything because its not done out in triage. They [the patients] are brought back to the prompt care area from the waiting room, if there's room available, but it's obtaining the history, vitals, and all that it's done in the prompt care, so that's the responsibility of the prompt care nurse and if she doesn't have a tech that day it's even less and it's on her and the mid-level will help sometimes. It's hard to be in the urgent care especially if you're the only nurse or sometimes they have been just one nurse or even being two because you have to do everything for the patient, not that you shouldn't check, double check what's been done, but you have to go in get the history, medicate, document on every patient that comes through there as opposed to 
working in the main ER you get your four or five patients that's your responsibility. Over there the flow is so quick you have to document on everyone that comes in, you can't just say client needs a set of vitals. (Lain)

Policy. Pediatric pain management is a shared responsibility among the ED

providers. The uses of nurse-physician joint protocols were used to empower nurses with a degree of autonomy that the nurse may implement pharmacological intervention to a pediatric patient while waiting to be seen by a mid-level or physician. The following is a policy for pain treatment for the ED patient:

Purpose: Provision of pain treatment protocols for the pediatric non-urgent patient. Scope: All Emergency Department Physician and Nursing Staff

Text: Non-pharmacological protocol

Cold and elevation for musculoskeletal and soft tissue injuries $<72$ hours old Immobilization of suspected fractures in position of comfort using slings and splinting Cleansing and dressings Distractions as adjunct to pain interventions such as relaxation techniques, reading materials, games, or television.

Non-urgent pain protocol for pediatric patients

Obtain vital signs and allergies

Administer Tylenol (acetaminophen $15 \mathrm{mg} / \mathrm{kg}$ ) if not taken within 4 hours PTA [prior To arrival]

If Tylenol taken less than 4 hours PTA and pain level greater than 4, administer Motrin $10 \mathrm{mg} / \mathrm{kg} P O$

If obvious skeletal deformity,

Children ages 3-6 years old administer Tylenol with codeine elixir $5 \mathrm{ml} P O$

Children ages 7-10 years old administer Tylenol with codeine elixir $10 \mathrm{ml} P O$ Repeat pain assessment and vital signs after one hour If pain level greater than 4 one hour after Tylenol or Tylenol with Codeine administration, consult physician.

Contraindications to analgesics

Allergy or sensitivity to the medication

Liver or kidney conditions/failure

Hemodynamic stability

Chest pain or abdominal pain

Multisystem trauma

Pregnancy; consult physician

Gastric disease

Coumadin (warfin)

Altered level of consciousness

Intoxication or suspected substance abuse (acute) 


\section{Lung disease with poor oxygenation/aeration}

Dependent on what providers were on, or if the unit was short staffed, or during

times of high volume, it was common to see basic comfort measures not given. The

following field note describes the care of a child in the prompt care area:

19:50 A young boy is registered in triage for hurt leg and ankle

20:50 The technician goes to the waiting room and calls in the boy. The boy comes in limping favoring his right leg. The patient goes to sit on the stretcher and the mother sits in a chair to the bottom left of the stretcher. The tech comes in to take the temperature under the tongue. The mother gets up and stands to the opposite of the stretcher. She shares with the tech that he is afraid of needles. "You can't be afraid, you're an Eagle fan" the tech reaches for the call bell/TV remote turns on the TV and looks for a show. He finds race cars, "nothing without cars going 102 miles an hour," the boy smiles. The physician walks into the room and speaks with the mom the boy points to his knee.

21:08 The boy returned from $x$-ray ambulatory and limping

22:40 The nurse looks over to the room with the boy, "I haven't even seen that kid."

23:00 The nurse walks in to apply an ace wrap and discharge the boy. (Field Notes, 04/30)

In this particular case, basics such as a wheelchair to remove weight bearing from the affected leg, and the use of cold compresses to the injured area were not implemented.

Some participants were quick to blame the physician for the under medication of children in pain as evidenced by the following remarks:

Well I think the doctors really under treat the kids' pain, if a kid comes in with a broken arm, umm they're always whisking them off to $x$-ray and you know, not thinking about, you know, they're always afraid to medicate kids, I don't know, the kids crying, the mother's upset, and they just seem to under medicate these kids, where I think these kids with the broken arm or deformities, or dislocations, you know require as much as an adult to sometimes get out of pain. They seem to forget that a lot of times with the kids. (Lizza)

While the participant in this interview pointed to the fact that the physicians' under medicated children, the policy documented above allowed initiation of a diagnostic and therapeutic plan by the nurse. Some participants in this study did not routinely administer pharmacological intervention for extremity injury, but did order extremity x-rays for 
children. Majority of the time if was not until a child had already returned from x-ray and had a painful extremity manipulated that pharmacological intervention was provided.

The emergency department had a 5 hour and 33 minute wait with 22 patients waiting to be brought into the department. A 6-year-old fell of a pony and in triage an $x$-ray was ordered. The patient was called back by the radiology technician and walked in with a visible red face with tears sobbing and guarding her arm. The child is brought back from $x$-ray. A verbal report is given by the technician to the mid-level there is a positive fracture. The radiology technician places the child in the chairs area of prompt care. There is an outstanding order for pain medication in the computer for the nurse. The mid-level did not share with the nurse the radiographic findings of the patient or reinforce that there was something ordered for pain in the computer. The mid-level explains to the parents that they are waiting for orthopedic.

Mid-Level (physician assistant): Did anyone call ortho?

Nurse: I will call ortho in a minute.

Tech: (Speaking to the child) You can come in this room and I am going to splint. The child is placed on the stretcher and accompanied by her parents and grandmother. The technician uses gauze to measure for the splint and goes to grab the supplies and returns. The technician kneels on the floor and begins to gently wrap the child's arm with gauze.

Tech: Don't move, sorry. One more roll, so far so good.

Child: This is KILLING ME!

Tech: This is the last roll. The child screams and cries.

Mom: Can we get something for pain or something?! The mom appears frustrated.

Tech: I just put on the splint, the nurse gives the medication. The technician does not stop manipulating the arm and reaches to place the sling on the child, "MY BONE, MY BONE!" The child screams and cries some more.

Tech: I'm just putting on the sling. After the sling was placed the technician walked over to the nurse and asked if that nurse had the patient. The nurse prepared the medication and then administered it to the child. (Field Note, 04/25)

External influences refer to outside social settings that indirectly influence the care of pediatric procedural pain on the ED unit. External influences included the culture of the overall hospital system, and accreditation institutions.

Patient Satisfaction. The role of organizational culture and leadership was an important indirect factor. A core philosophy of Fellowship Hospital was that growth begins and ends with patient satisfaction. Survival of Fellowship Hospital has been 
contingent on the idea that patient satisfaction has become both the means as well as the

ends.

Fellowship Hospital's goal has always been to deliver outstanding health care with a high level of patient satisfaction to people living and working in the surrounding communities. Recognizing the importance of patient satisfaction to our continued success, Fellowship set a goal to differentiate itself in terms of service excellence. In order to fuel this initiative and create a "burning platform" for change patient satisfaction is on the agenda of each meeting from board level to employee forums. Although there are many reasons why we have enjoyed the success that we have, number one among them has been absolutely uncompromising commitment toward patient satisfaction. It's a philosophy that touches every aspect of this hospital. ( News Article, 2001)

Emergency service was one of the four key areas measured for patient satisfaction. The ED was described as the community's front door to the hospital with over $70 \%$ of inpatient admissions initiated through the ED. Thus, ED performance was selected as an area that fueled growth and accommodated the communities' needs with a resultant major expansion. The emphasis on creating customer loyalty applied an external pressure on participants based on the importance of patient satisfaction scores with regard to the care as it was perceived by the patient. Key healthcare services and processes with regard to the ED focused on triage to EKG time, patients who left the ED without treatment, unplanned returns within 72 hours, ED patient satisfaction with nursing care, and ED patient satisfaction with physicians. (Hospital Document, 2003)

Previously, a key indicator of ED dissatisfaction was the number of patients who left without treatment. This issue was dealt with through the redesigned triage process to include more staff during high volume times in the ED and the placement of a mid-level in triage to expedite the assessment and treatment of patients in the waiting area. To reinforce the importance of patient satisfaction, weekly scores were posted on staff informational boards. 
Week 11 Patient Satisfaction 95\%

Patient Satisfaction Week $16=99 \%$

Week 25 Press Ganey Scores ED 84 lowest of all the departments

Congratulations to Emergency Department Overall Score 92\% week 26

Top 10 Priorities in the Emergency Department:

1. Staff cared about you as a person

2. Information about delay

3. Overall rating of ER care

4. Likelihood of recommending

5. How well pain was controlled

6. Waiting time to see doctor

7. Adequacy of information family and friends

8. Nurses informative regarding treatments

9. Courtesy shown to families/friends

10. Waiting time to treatment area

Areas that showed improvement were waiting time to see doctor, informed about delay, nurse concerned for privacy, and nurses attention to your needs.

Negative areas:

How well pain was controlled - 3.9

Let family and friends be with you -2.6

Staff cared about you as a person -3.0

Information about home care -3.4

Helpfulness of first person - 4.6

Waiting time before notice arrival -5.2 (Field Notes, 04/11)

Press Ganey. Participants emphasized that the quality of care was never affected in the ED just the flow of patients; however the results of these ratings from patient satisfaction scores would contradict those statements. A disconnect existed between what the participants thought was care and what the patients and/or family members believed to be care. In part, accountability and focus of priorities at Fellowship Hospital is set highly on patient satisfaction. Press Ganey, the nation's leading healthcare satisfaction measurement firm is one of the many benchmarks used by Fellowship Hospital. The influence of Press Ganey patient satisfaction scores is illustrated below:

Researcher: One of the nurses pointed out that the cliental is changing, what does that mean? 
Mid-Level (physician assistant): It means that patients who don't get their drugs at hospital $X$ or $Z$ come here, and we are all about customer service. We give them prescriptions and score better on Press Ganey.

Researcher: The physician on today does not practice like that.

Mid-Level: He's part-time the full timers stress it, because if you don't they [the patients] tend to rate us low on Press Ganey and scrutinize our work. (Field Notes, 06/05)

The importance of scoring well on Press Ganey was also due to the fact that there were four acute care hospitals within a 15 mile radius that competed for both patients and quality healthcare professionals. In New Jersey it has been a critical time for hospitals, since 1992 to 2009 twenty-five hospitals have been closed, and since 2007 six more hospitals have filed for bankruptcy (New Jersey Hospital Association, 2010). Refer to Appendix P for list of hospital closings.

While other hospitals were closing, downsizing, or decreasing the amount of services offered Fellowship continued to expand the amount of services offered to their customers.

This system creates a culture where the customer drives our actions and aligns the organizations objectives with customer requirements. By maintaining a focus on creating loyalty within the customer group Fellowship has experienced exponential growth, and has attained market leader status in an extremely competitive environment. (Hospital Document)

In the moments that participant's spent with patients they practiced to the best of their abilities, but what should be practice of caring has now been subsumed into patient satisfaction. Comforting and caring was promoted as a means to increase patient satisfaction scores it was a concept for all employees within the hospital setting not limited to nursing. The following was printed in Fellowship News:

Here are some patient satisfaction tips to help you contribute to a positive experience for patients and families: Avoid using sweetie or honey

Introduce yourself and explain what you'll be doing 
Be aware of facial expressions and non-verbal language

Show concern for the patient's comfort by asking if there is anything that can be done to make them more comfortable

Make eye contact and smile

Communicate and remember that listening is part of the process

Be sympathetic to the patient and try to put yourself in their shoes sometimes they just need to vent and have someone listen to them.

Take five or ten minutes and listen to whatever it is they need to talk about. It can make a big difference.

For participants process improvement (PI) was another system approach by

Fellowship Hospital to monitor performance. A document posted on the staff

informational board was used to clarify for ED providers the role of PI.

Commonly Asked Questions:

Q: What kind of PI indicators does the emergency department monitor?

A: We monitor transfers, medication reconciliation, moderate sedation, pain, restraints, acute observation, patient safety behaviors, and more.

Q: How does the emergency department chose what to monitor?

A: We monitor based on high risk, low volume, by sentinel events or organization priorities.

Q: What does the department do with the PI results?

A: PI results are shared within the department and with hospital leadership.

Educational and operational initiatives are created with input from the PI results. PDCA format for process improvement plan, do, check, act. (Field Notes, 05/07).

The Formulated Plan to address, "How well pain was controlled":

1. Rounding to include pain assessment (The Rounding Program includes hourly rounding focused on "Checking on your PEPPs"; pain, environment, position, and potty).

2. Work with triage and medical staff to ensure patient in waiting area have pain addressed.

3. Re-inservice emergency department pain policy.

4. Response to actionable items to be monitored weekly by Press Ganey Reporting (Field Notes, 04/11)

Process Improvement PI:

Pain Initial Assessment March 2011 49\%

February $201138 \%$

80 charts per month reviewed

Pain reassessment after medication administration 
March $201168 \%$

February $201198 \%$

80 charts per month reviewed (Field Notes, 05/07)

Accreditation Organization. Accreditation organizations represent the state, New

Jersey Department of Health and Senior Services (NJDHSS), and federal, Joint

Commission formerly known as Joint Commission on Accreditation of Healthcare

Organizations (JCAHO) institutions. NJDHSS and JCAHO were institutions located

outside the emergency unit, and hospital that exerted a degree of influence on the

practices of healthcare providers in the treatment of children in pain. Fellowship as a

business is proud to have received full standards compliance from the Joint Commission.

As a provider of healthcare services, Fellowship operates under a strict legal and regulatory environment...there are numerous standards that are required of healthcare organizations to ensure quality care, fair and equal treatment, ethical business practices, confidentiality and safe environment. (Hospital Document)

Adherence to NJDHSS and Joint Commission standards were important to

maintaining Medicare certification, liability insurance, and state licensure requirements.

Activities in the hospital changed based on anticipation related to potential visits from

these agencies.

The prompt care area is very quiet and slow there are five personnel in the area between zone 3 and prompt care. One of them is a woman wearing a jogging suit[nursing leadership in for the weekend to prepare the ED]. She has plastic gloves and is busy cleaning the counter tops and pulling materials that are not dated or are outdated. The educators pin up board that is usually cluttered with papers pinned on top of each other was cleared of all the papers except for a colorful array of push pins. An infant crib that was normally just left unattended to in the hallway next to the staff lounge was wiped down, draped with a white sheet, and an infant appropriate resuscitation bag was placed at the bottom. The infant warmer too was also cleaned and draped over with a sheet. (Field Notes, 05/21)

Please join us for the Joint Commission Readiness Fair to learn more about Joint Commission Standards and the National Patient Safety Goals. Attendance is Mandatory for all staff.

Located at the fair the Joint Commission Board on Pain: (Joint Commission codes 
are assigned to each answer)

Q: When is a patient assessed and reassessed for pain?

A: Pain is assessed upon admission to hospital and outpatient areas. Pain is reassessed on the hospitalized patient at least every shift, before and after pain medications.

Admission in the appropriate time frame $1 / 2$ hour for IV 1 hour for $P O$.

Reassessment of the outpatient will be conducted as appropriate to the reason the patient is presenting for care and services before and after medication

administration. Please don't forget to chart, take credit for all the good thing you do. PC.01.02.07 EP3: The hospital reassess and responds to the patient's pain based on its reassessment criteria.

Q: What pain scales do we use to assess our patients?

A: Likert numeric 1-10

FLACC: Non-verbal/impaired patients

Wong-Baker Faces Scale

PC.01.02.07 EP1: The hospital conducts a comprehensive pain assessment that is consistent with its scope of care, treatment, and services and patients conditions.

Q: What are the components to the hospitals pain assessment?

A: The hospital uses methods to assess pain that are consistent with the patient's age, condition, and ability to understand.

PC. 01.02.07 EP2: The hospital assesses and manages the patients in pain.

(Field Notes, 06/11)

Documentation of pain by participants followed a predictable pattern addressing the

Joint Commission Standards. The use of computerized charting provided standardized

prompts for participants to check off and click as appropriate. The ED was considered an outpatient unit therefore policy written for the treatment and documentation of pain for the outpatient area was followed. Fellowship Hospital respected and supported the patient's right to pain management.

The hospital recognizes and supports the adequate management of pain as a patient right. The goal of pain management is to improve the quality of life of patients experiencing pain by providing relief for those is acute and chronic pain through assessment, reassessment and pain management techniques.

The treatment of procedural pain was briefly addressed in a short paragraph located in the general policy for the management of the patient with pain: (Refer to Appendix Q) 
Healthcare providers will consider any treatments or procedures for their potential to cause pain and plan care appropriately. Plan of care will include the patient/family when possible and consider alternative treatment modalities, spiritual/cultural and life cycle needs of the patient as appropriate. When indicated, the Licensed Independent Practitioner will be notified of alterations in pain or inadequate pain relief from ordered interventions.

No formal policy was located for the treatment of procedural pain in pediatric patients specific to minor needle procedures. This may be due to the non-specific broad pain management standards set by the accreditation agencies that appear to drive the culture of pain practice in this institutional setting.

\section{Summary of Environmental Issues and Policies}

For the participants in this study, environmental factors that directly inhibited the optimal pain management of pediatric patients consisted of keeping the flow, and time management preparing for uncertainty. The practice of pediatric pain care was also influenced by organizational and unit policies, patient satisfaction as a driver for excellence, Press Ganey, and accreditation requirements. 


\section{Chapter VI}

\section{Discussion of Findings}

In a qualitative study that uses ethnography as method, the findings are written as stories and narratives that emerge from the data. In this study, pediatric pain management beliefs and practices of health care providers in a non-pediatric ED were described. For this chapter, the findings are discussed in relation to the extant literature and research assumptions are revisited. The literature presented will substantiate the findings and provide insight to the relevance and significance of this study to the current knowledge base.

\section{Research Assumptions}

The first assumption of this study was that social factors represented defining features of human environments and were a powerful source of variation in how the emergency department operated. This assumption proved to be true. While participants in this study were aware of other pharmacological interventions to assist children during painful procedures such as the use of topical anesthetics for intravenous insertion and the use of benzodiazepines for more lengthy invasive procedures, they deferred to the fact that those types of things were not done on the unit, and that physician practices dictated the manner children were pharmacologically managed during painful procedures. Many of the techniques that medical technicians and nurse assistants used in their practice were referred to as tricks of the craft learned from nurses on the unit. The priority of managing pediatric procedural pain was a direct reflection of the tone set by medical and nursing leadership on the unit and the hospital organization. 
The second assumption of the researcher was that emergency staff would be focused on carrying out the technical aspects of children's care, and comforting children would not be a major activity. This assumption did not apply to all participants. While some participants were very task oriented and focused on the delivery of the more practical aspects of care, the degree of comfort given to a child was highly dependent on the healthcare provider on duty. Those participants who expressed a want and desire to work with children were more apt to incorporate a child developmental approach.

The third assumption of the researcher was that pain assessment of preoperational children was based on nurses' intuition of what the child was experiencing without the use of a standardized method to interpret and quantify pain behaviors. This too proved to be highly individualized and variable. While nurses were observed to ask children to rate their degree of pain, those same nurses' with a different patient would not ask about pain but assign a pain intensity score on the patient record. A nurse was more likely to assign a pain intensity score for conditions deemed painful or if a child displayed more overt signs of pain and distress. No participant during observation specifically mentioned the use of a FLACC scale when assigning the pain score, one participant was very specific and measured pain intensity of the child by looking at the child's facial display and compared it to the FACES scale. There was no consistency in the use of pain measurement scales, from one provider to the next with the same patient. For example, a numerical scale may have been used in triage to assess the patient, and then a FACES scale used at the bedside. The measurement of pain intensity during painful procedures was not observed as a part of any participants practice. 
The fourth assumption of the researcher was that simple non-pharmacological methods such as distraction would not be used with any regularity. This proved to be false. Distraction, parental presence, and especially reassurance and other techniques were used to assist children during procedures that caused pain or distress. No formal education by the healthcare provider to the caregiver was given on how to distract or reassure the child, there was no designated person in the room responsible for the distraction intervention. For example, when three or four people were observed in a room, caregivers and healthcare providers, different distractions were offered to the child.

The fifth assumption of the researcher was that healthcare providers in the nonpediatric ED would have a negative and non-supportive attitude toward pediatric procedural pain management. In this study, all participants acknowledged that children experienced a certain degree of distress during the procedure; however participant beliefs were highly variable as to the potential for long term consequences related to brief exposure of procedural pain. Participants perceived a hierarchy of pain dependent on the type of injury, diagnostic, or therapeutic procedure performed. For example, participants acknowledge the need for pain management of burns, and fractures but minimalized the need for minor needle procedures.

The sixth and final assumption of the researcher was that human behavior was shaped in the context of a sociocultural milieu and that every human event was culture-bound. This proved to be true. Participants were aware of the existence of pain management policies to address children's pain in the ED unit; however, in this study delays in analgesia administration were seen during times of high patient volume, crowding in the waiting room, staff shortage of nurse and technicians, and simultaneous competing 
priorities for healthcare providers attentions. Patients were also found to be less likely to receive basic comfort measures such as ice for musculoskeletal sprains, and rewards (e.g. teddy bears) after a procedure, if there was a high volume of patients waiting to be seen.

\section{Relationship of the Findings to Extant Literature}

A review of the literature using PubMed, CINHAL, Medline and PsycLit was undertaken to identify published work as recent as January 2012 relevant to the domain of pediatric procedural pain assessment and management in the ED. Relevant themes identified in this study also guided the literature review. Themes in this literature review include: pediatric pain assessment in the $\mathrm{ED}$, procedural pain in the $\mathrm{ED}$, communication related to pediatric comfort in the $\mathrm{ED}$, treatment of pediatric procedural pain in the $\mathrm{ED}$, attitudes of health care providers toward pediatric procedural pain in the ED, environmental issues and policies related to pain management in the ED.

\section{Theoretical Frameworks: Pain Management in the ED}

A variety of theories and conceptual models have been suggested to explain pediatric pain management (Broome \& Huth, 2003; Cohen et al., 2007; Huth \& Moore, 1998); however, the continued under treatment of pain in the ED indicates that the root cause for under treatment of pain in the ED may not be well understood (Johnson, 2005). The practice of emergency medicine and nursing has been identified as a unique specialty practice (Emergency Nurses Association, 2011; Johnson, 2005); therefore, factors that may contribute to the under management of pediatric pain in the ED may also exist.

Two conceptual models were located in the literature specifically developed to guide research and address pain management issues in the ED setting (Russo, 2010; Tanabe, 1996). Tanabe (1996) developed a conceptual model, "Variables Affecting Pain in the 
Emergency Department", to specifically address pain management issues in the ED setting. The model consisted of system variables and patients variables. System variables included treatment of pain, knowledge and attitudes of health care workers, and emergency department environmental stress levels. Patient variables included physiologic, psychological, and demographic variables.

Russo (2010) using a heuristic approach formulated the logic model for triage decision-making and pediatric pain assessment. The model served to provide an understanding for the interactive layers of context in which the triage nurse made decisions regarding the child who presented to the ED with a painful diagnosis. The model consisted of child and contextual variables. Child variables included: Age, gender, ethnicity, behavior, symptom, appearance, and the interaction of parental behavior. Contextual variables included: The physical setting as the type of ED, the population, the volume of patient visits to the ED, staffing patterns of the triage area, and the availability of pediatric specific equipment, assessment tools, and technology. Both models proposed were limited to the assessment and management of pain as a chief complaint in ED patients, and did not address procedural pain. Of the two ED specific studies, only one examined pain assessment and management of pediatric patients. Tanabe (1996) examined the knowledge and practices of emergency nurses related to pain assessment and management of patients 18 years and older. Russo (2010) focused on pain assessment and management of pediatric patients limited to triage nurses'.

\section{Pediatric Pain Assessment in the Emergency Department}

A majority of the literature on pediatric pain assessment in the ED is focused on children who present to the department with painful diagnosis. Previous studies on 
pediatric pain assessment in the ED setting have primarily measured acute pain as a chief complaint (Alexander \& Manno, 2003; Chisholm et al., 2008; Crocker, Higginbotham, King, Taylor, \& Milling, 2011; Fry et al., 1999; Garra et al., 2009; Gourde \& Damian, 2012; Johnston et al., 1998; Kelly, Powell, \& Williams, 2002; Maciocia et al., 2003; McConahay, Bryson, \& Bulloch, 2006; O’Donnell, Ferguson, \& Bettie, 2002; Probst et al., 2005; Rajasagaram, Taylor, Braitberg, Pearsell, \& Capp, 2009; Russo, 2010; Santervas, et al., 2010; Shavit, Kofman, Leder, Hod, \& Kozer, 2009; Strout \& Baumann, 2011). The most common method to identify pain assessment practices in the emergency department has been through survey and retrospective chart review (Alexander \& Manno, 2003; Jadav, Lloyd, McLauchlan, \& Hayes, 2009; Kaplan, Sison, \& Platt, 2008; Kleiber, Jennisen, McCarthy, Ansley, 2011; May et al., 2009; Porter \& Chapman, 2004; Probst et al., 2005; Santervas et al., 2010; Strout \& Baumann, 2011); however, healthcare providers' have been found to report better pain assessment practices on surveys then when compared to actual practice (Read, 1994; Twycross, 2007b; Twycross, 2008).

As previously mentioned, one study was located that used observational methods to investigate observed ED physician pain assessment and documentation in the medical record. Chisholm, Weaver, Whenmouth, Giles \& Brizendine (2008) observed bedside interactions of emergency physicians and found that physicians acknowledged the patient's pain in $98.1 \%$ of the time but documented the presence of pain in $91.7 \%$, physicians attempted to quantify the patient's pain in $61.5 \%$ of encounters but documented that attempt 38.9\%. Chisholm et al. (2008) also noted instances where physicians' made no inquiry about the quantity or presence of pain, yet documented pain and pain intensity on the patient chart. This finding was similar to participant practices in 
the current study where pain intensity scores both numerical, and FACES were documented on the medical record when neither parent nor child were verbally asked to rate the pain.

The overall documentation rates of pediatric pain measurement in the ED setting remains low (Drendel et al., 2006; Kaplan et al., 2008; Kleiber et al., 2011; Porter \& Chapman, 2004; Probst et al., 2005). Two national surveys were located with one focused on pediatric pain assessment in the ED setting, and the other on pediatric pain assessment practices in teaching hospitals. Drendel, Brousseau, \& Gorelick (2006) conducted a cross-sectional analysis of ED visits for pediatric patients from the National Hospital Ambulatory Medical Care Survey 1997 to 2000, out of 24, 707 visits only $44.5 \%$ of visits had documented pain scores. The authors concluded that the younger the age of the patient the less likely pain score documentation was recorded. This finding was previously supported from the work of Broome, Richtsmeier, Maikler, \& Alexander (1996) who examined how health care providers in United States teaching hospitals assessed children's pain, out of 113 respondents from institutions with pediatric residency programs $27 \%$ reported no formal self report scale, $20 \%$ reported the use of behavioral scales, $73 \%$ reported using some type pain scale, and pain measurement tools for younger children were perceived to be less effective pain measurement tools. The results of both national surveys were conducted prior to the implementation of JCAHO standards. Recent studies have reported that the implementation of mandatory pain documentation has increased the rate of documentation for pain, but to have minimal or no effect on analgesic administration for the pediatric ED patient (Jadav et al., 2009; 
Kaplan et al., 2008; May et al., 2009; Santervas et al., 2010). The observed documentation rate for participants in the current study varied from 60-70\%.

Literature on the measurement of children's procedural pain in the ED setting was limited for use during clinical studies that attempted to validate pharmacological, nonpharmacological, or multimodal interventions (Adriansson, Suserud, \& Bergbom, 2004; Babl, Mandrawa, O’Sullivan, \& Crellin, 2008; Carlson et al., 2000; Cavender, Goff, Hollon, \& Guzzetta, 2004; Kissoon, McGrath, \& Glebe, 1990; McErlean, Bartfield, Karunakar, Whitman, \& Turley, 2003; Schultz, Strout, Jordan, \& Worthing, 2002; Sinha, Christopher, Fenn, \& Reeves, 2006). No studies were located that documented practice patterns for pain measurement of pediatric patients during painful procedures in the ED setting.

\section{Pain Assessment Patient, Provider, Parent}

Results from previous studies show that healthcare provider's perceptions are poor indicators of pain for the pediatric patient in the ED (Maciocia et al., 2003; Rajasagaram et al., 2009; Singer, Gulla, \& Thode, 2002; Shavit et al., 2009). Pain is subjective therefore it is important that the patient's perception of their own pain is assessed (International Association for the Study of Pain, 1979; Kelly et al., 2002; Shavit et al., 2009; Zempsky, Cravero, American Academy of Pediatrics Committee on Pediatric Emergency Medicine and Section on Anesthesiology and Pain Medicine, 2004). The results of one study where the phenomenological approach was used revealed emergency nurses utilized their observations of behavior to estimate pain in young children (Russo, 2010). Russo (2010) interviewed ED triage nurses as part of a mixed method design, major themes that emerged from the data included: Really looking at the patient and 
behavior can tell a lot. Triage nurses described how they improvised the use of pain scales for young children by estimating a pain score through observation of behavior; concerns were related to the child's limited ability to understand the pain scale.

In a different study, Shavit, Kofman, Leder, Hod, \& Kozer (2009) compared observational measurement scores and self-report pain scores in children aged 3 to 15 years of age who presented with a chief complaint of pain. In this study, ED triage nurses' used an observational assessment tool (the Alder Hey Triage Pain Score), the findings revealed that the scores on nurses' observational assessment tool were lower than the patient's self-report. This finding was similar to the poor correlation between adult patient and practitioner assessments of pain in the ED (Fry et al., 1999; Guru \& Dubinsky, 2000; Puntillo et al., 2003; Singer et al., 1999).

Previous research has examined the psychological effect of anchoring, a phenomenon that occurs when observers over rely on initial impressions, and subsequently discount relevant disconfirming evidence. Riva, Rusconi, Montali, \& Cherubini (2011) sampled 423 physicians, nurses, medical students, and nursing students, used computerized vignettes about hypothetical patients who arrived in the emergency room with a wart, and during the visit complained of a headache. The researchers found that healthcare providers anchored to initial impressions in light of the patient's pain ratings, $48 \%$ of the respondents fully anchored to their initial impression of pain intensity, and $87 \%$ of the sample were influenced by bias toward the initial impression, thus insufficiently adjusting their pain judgment.

Studies that examined parental perceptions of pediatric pain in the ED versus child's self-report have reported conflicting results. Two studies concluded no significant 
difference in pain scores between the parent and child (Maciocia et al., 2003;

Rajasagaram et al., 2009), and two studies reported parents tendency to under estimate the child's pain (Kelly et al., 2002; Singer et al., 2002). Results of studies regarding the reliability of parental perception of pediatric pain in the ED remains mixed.

\section{Reliability of Pain Instruments in the ED}

The assessment of pediatric pain has been theorized as the first step to the appropriate treatment and management of pain (Broome \& Huth, 2003; Huth \& Moore, 1998). Observation of behavior has been recommended to complement self-report particularly for toddler and preschooler children whose verbal skills remain limited and quite variable (American Academy of Pediatrics \& American Pain Society, 2001; Craig, Lilley, \& Gilbert, 1996; Herr et al., 2006; Herr, Coyne, McCaffery, Manworren, \& Merkel, 2011). Researchers have raised concerns about the reliability, validity, and clinical practicality of pain measurement tools in the ED setting (Belville \& Seupaul, 2005; Crowley et al., 2011; Drendel et al., 2006; O'Donnell et al., 2002; Terndrup, 1996). Porter and Chapman (2004) reported that nurses in an Australian ED did not appropriately utilize pain assessment tools for the pediatric patient and Russo (2010) found that nurses improvised the use of pain scales with younger pediatric patients. Various pain measurement instruments are available for use with pediatric patients in the acute pain setting; however there is no accepted criterion standard and few have been psychometrically examined in the ED unit (Bishop-Kurylo, 2002; Strout \& Baumann, 2011). Six studies were located that evaluated different pain measurement tools in the pediatric ED (Bulloch \& Tenenbein, 2002; Crellin, Sullivan, Babl, O’Sullivan, \& Hutchinson, 2007; Garra et al., 2009; Schultz et al., 2002; Stewart, Lancaster, Lawson, 
Williams, \& Daly, 2004; Strout \& Baumann, 2011). Of those studies three focused on acute pain assessment associated with chief complaint (Bulloch \& Tenenbein, 2002; Garra et al., 2009; Stewart et al., 2004), one study evaluated the psychometric properties of the modified version of the pre-verbal, early verbal, pediatric pain scale (M-PEPPS) via secondary analysis from data collected on pediatric patients 12 months through 84 months however data on the nature of pain whether procedural or as a chief complaint was not identified (Strout and Baumann, 2011).

Schultz, Strout, Jordan, and Worthing (2002) explored the usefulness, reliability, and validity of the M-PEPPS on a convenience sample of children between 12 and 84 months who underwent peripheral intravenous catheterization in the ED. M-PEPPS demonstrated high interrater reliability, high internal consistency, and good concurrent validity with children's self-report scores versus parents' perceptions of their children's pain as measured by the VAS,

Only one study systematically reviewed the literature to identify validated preverbal and early verbal behavioral pain and distress scales (Crellin et al., 2007). Crellin, Sullivan, Babl, O’Sullivan, \& Hutchinson (2007) analyzed six major behavioral pain and distress scales that included the Children's Hospital of Eastern Ontario Pain Scale (CHEOPS), Face Legs Activity Cry Consolability Pain Scale (FLACC), Toddler Preschooler Postoperative Pain Scale (TPPPS), Preverbal, Early Verbal Pediatric Pain Scale (PEPPS), Observer Visual Analog Scale (VAS), and the Observation Scale of Behavioral Distress (OSBD). The authors selected these scales because they had been previously validated in the procedural setting as pain or distress tools, or had been validated in the nonprocedural setting and either used to publish research or could be 
potentially used to assess procedural pain and distress in the preverbal and early verbal population. The authors concluded with two recommendations, the CHEOPS and FLACC scales as potential valid measures of procedural pain for clinical practice and research in the ED, and the need for further validation studies of behavioral pain and distress tools for pre or early verbal children undergoing painful procedures in the ED (Crellin et al., 2007). Standard self-report scales have been reported to be reliable and valid for children 3 years and up; however standardized scales have not been designed specifically for the assessment of pain in the ED setting and there is a lack of data on whether those children who can validly use pain scales can be identified beforehand (Besenski, Forsyth, \& von Baeyer, 2007; Shavit et al., 2009).

\section{Use of Experts and Physical Restraint}

Expert technical skill has been recommended in clinical practice statements to be an important facet to procedural pain management (American Academy of Pediatrics \& American Pain Society, 2001; Jeffery, 2002; Zelter, Jay, \& Fisher, 1989). In a joint statement released by the American Academy of Pediatrics and the American Pain Society (2001), procedures should be performed or directly supervised by healthcare providers with sufficient technical expertise in order to minimize pain to the greatest extent possible. The rationale for the use of an expert was to limit the duration and invasiveness of the procedure, thus minimizing the degree of stress to the child and family (Jeffery, 2002). No articles were located that empirically explored the relationship between healthcare provider technical skill during procedures in the ED and the effect on children's self-report of pain. 
In contrast, various articles were located that discussed the use of restraints in the clinical care of adult patients in the psychiatric setting, in the intensive care unit, and care of the elderly (Benbenbishty, Adams, \& Endacott, 2010; Brenner, 2007b; Hine, 2007; Kontio et al., 2010; Lewis, Taylor, \& Parks, 2009; Saarnio \& Isola, 2009; Tested, Aasland, \& Aarsland, 2005) . The existing reports on pediatric restraint were largely limited to the psychiatric setting, practice and attitudes of pediatric nurses, position statements, ethical issues, and clinical practice recommendations (American Academy of Pediatrics, 1997; Bland et al., 2002; Demir, 2007b; Dorfman \& Kastner, 2004; Dorfman \& Mehta, 2006; Emergency Nurses Association, 2004; Hull \& Clarke, 2010; Jeffery, 2010; Lambrenos \& McArthur, 2003; Pearch, 2005; Mohr, Petti, and Mohr, 2003; Royal Australasian College of Physicians, 2006; Selekman \& Snyder, 1997; Tomilson, 2004; Valler-Jones \& Shinnick, 2005).

Brenner (2007b) conducted a review of the literature on child restraint and concluded there was a dearth of research in relation to the use of restraints in pediatric nursing, most articles advocated awareness and encouraged the use of alternative practices. The use of physical restraint has been identified as a common accepted practice used in pediatrics to control the movement of the patient during procedures (Bland et al., 2002; Collins, 1999; Demir, 2007b; Jeffery, 2002; Selekman \& Snyder, 1996). The use of restraint has been an accepted practice during common procedures such as immunization, intramuscular injection, venipuncture, intravenous line placement, and lumbar puncture (Bland et al., 2002; Collins, 1999; Robinson \& Collier, 1997).

Robinson and Collier (1997) surveyed 394 pediatric nurses in the United Kingdom; to identify factors that influenced nurses' decisions to hold children and to find out how 
often children were required to be held still during medical procedures. Of the 153 respondents, $69.3 \%$ reported that restraint was the main cause for distress during procedures, and $13.7 \%$ reported pain as the main cause of distress. Seventy-four percent reported that the main reason for holding a child still was to avoid accidental injury and protect the child. With regard to formal training, $16.6 \%$ reported that they had received formal training on holding techniques and $90.8 \%$ of respondents believed guidelines on how and when to hold was necessary. The authors concluded that restraining children during clinical procedures was common in nursing practice, but a neglected topic of research due to the taboo nature of the issue.

Physical restraint of children was considered an uncontested practice (Collins, 1999) and the use of such practices had been justified on the premise that nurses acted in the best interest of the child. Other authors have reported the reasons for restraint use in children were to prevent treatment interference (Brenner, 2007a; Graham \& Hardy, 2004; Tomilson, 2004), necessity of the procedure, to maintain stability, safety of the child, and the safety of others (Jeffery, 2002; Kennedy \& Mohr, 2001; Robinson \& Collier, 1997; Tomilson, 2004). The term "brutacaine" was a term previously used by researchers to emphasize the frequency for the use of restraints on children. Brutacaine was defined as a tradition whereby children were wrapped up in a blanket with or without a premedication and suturing was done with the aid of local anaesthetic (McGlone, Ranasinghe, \& Durham, 1998).

In the literature reviewed, little has been written on the incidence or rationale for the use of restraint in pediatric patients in the emergency department setting. Of the three empirical studies located, restraint use was limited to psychiatric patients in the pediatric 
emergency setting (Dorfman \& Kastner, 2004; Dorfman, \& Mehta, 2006), and restraint of pediatric patients during radiographic examinations (Graham \& Hardy, 2004). No studies to date have examined aspects of pediatric restraint use during routine emergency department procedures.

\section{Treatment of Pediatric Procedural Pain in the Emergency Department}

Various guidelines and clinical practice statements are available in the literature related to the treatment of pediatric procedural pain in the ED setting (American Academy of Pediatrics \& American Pain Society, 2001; Crowley et al., 2011; Emergency Nurses Association, 2010; Kennedy \& Luhmann, 1999; Krauss, 2001; Ramponi, 2009; Young, 2005; Zempsky et al., 2004). In the literature reviewed, the implementation of pharmacological interventions for the management of painful procedures in ED practice has been relatively low. Only three studies were found that examined procedural pain management treatment patterns in the pediatric ED and in EDs of a rural state (Bhargava \& Young, 2007; Kleiber et al., 2011; MacLean et al., 2007).

Bhargava and Young (2007) surveyed fellowship directors of U.S. EDs with a pediatric emergency medicine training program, of the 51 pediatric fellowship programs surveyed $75 \%$ responded. All respondents reported some type of pain management (topical anesthetic most common), or sedative drug for laceration repair, $48 \%$ reported pharmacological method for sedation for cranial CT imaging, and $38 \%$ for intravenous catheter placement. The use of pharmacological intervention for venipuncture and intravenous catheter insertion were not regularly used by the majority of respondents.

Kleiber, Jennissen, McCarthy, and Ansley (2011) surveyed all licensed independent providers and nurses from the 118 hospital EDs in a rural state, of the 259 providers and 
1,177 nurse respondents both providers and nurses never or infrequently used pharmacological intervention before intravenous catheter insertion or venipuncture, $54 \%$ of providers and $48 \%$ of nurses reported using topical anesthetic for laceration repair 75 to $100 \%$ of the time, and $53 \%$ of providers and $50 \%$ of nurses used anesthetic ear drops for ear pain most of the time.

MacLean, Obispo, \& Young (2007) reviewed pediatric charts of children aged 0-17 years who presented to the pediatric ED during a 4-month period, over 6,545 patients were seen with 1,727 painful procedures performed on 1,210 patients. The authors found that pharmacological pain treatments were not routinely administered for minor procedures. None of the patients who underwent fingersticks, intramuscular injections, subcutaneous injections, or urethral catheterization received any documented pharmacological intervention for procedural pain, of the $13 \%$ of patients who underwent venipuncture or intravenous line placement less than $1 \%$ received topical anesthetic cream. In $84 \%$ of cases where fracture reduction was needed procedural sedation with ketamine was administered, $67 \%$ of those who needed laceration repair were managed with injected local anesthetic, $29 \%$ received topical anesthetic gel prior to injected local, and $12 \%$ received procedural ketamine.

The practice of non-pharmacological intervention use in the ED is not well documented in the literature, other than the use of non-pharmacological methods used during research trials there is no standard documentation on the medical record. Of those studies that examined procedural pain management practices in the ED, two studies surveyed respondents on practice patterns for non-pharmacological treatments. Kleiber et al. (2011) reported that approximately $50 \%$ of providers used distraction or taught 
parents interventions to assist children on how remain calm during painful procedures, more than $70 \%$ of nurse respondents reported the use of distraction, and more than $90 \%$ of both providers and nurses prepared children by telling them what would happen during the procedure. The practice of applying pressure at an intramuscular injection site before injection was reported to never be used by more than $50 \%$ of nurse respondents. Bhargava and Young (2007) categorized non-pharmacological interventions as parental support and child life; most respondents used parental support with child life during intravenous catheter placement (62\%) and lumbar puncture (20\%). MacLean et al. (2007) conducted retrospective chart review and were unable to report non-pharmacological interventions as those interventions were not routinely documented on the patients chart.

Four studies were located that evaluated the non-pharmacological intervention of distraction in the ED unit (Carlson et al., 2000; Sinha et al., 2006; Winskill \& Andrews, 2008; Young, Griffin, Phillips, \& Stanley, 2010). Of those articles, two studies utilized quantitative measures. Carlson, Broome, and Vessey (2000) examined the effectiveness and feasibility of distraction on reduction of child behavioral distress, self-reported pain perception, and fear during venipuncture or intravenous catheter insertion in thirteen pediatric EDs across the United States and Canada. Children aged 4 to 18 - years were randomly assigned to the distraction intervention or the attentional control group. The attentional-control group received verbal preparation of the provider's choice, instruction about the procedure, and comfort techniques normally used in practice. Participants in the experimental group received and were guided to use an Illusion Kaleidoscope throughout the procedure. The investigators reported no significant difference in self-reported pain or behavioral distress for children who underwent needle sticks in the ED setting. 
Sinha, Christopher, Fenn, \& Reeves (2006) randomly assigned children between 6 and 18-years of age visiting the emergency department for laceration repair to a control or intervention group by a patient allocation scheme implementing a stratified block design. All patients regardless of group assignment were evaluated and treated according to standard protocol that included the use of topical anesthetic gel, supplemental local anesthetic, and additional local anesthetic during the procedure as needed. Children in the intervention group were given a choice of age-appropriate distractors including music, video games, cartoon video, or a child life specialist offered to read a book or help of blowing bubbles during the procedure. Quantitative measure of pain intensity, situational anxiety, and pain distress as perceived by the parent were assessed by using the 7-point Facial Pain Scale (FPS), State Trait Anxiety for Children (performed on participants $\geq 10$ years of age, and a visual analog scale, before and after laceration repair. The findings of this study demonstrated no statistically significant differences for changes in the FPS scores between the 2 groups for children younger than 10 years. The results indicated that although the use of distracters did not reduce self-reported pain intensity in children during laceration repair, this intervention was effective in reducing self-reported anxiety associated with the procedure in older children during laceration repair in the ED. For younger children, pain distress was reduced by distraction as measured from parental perception. In conclusion, the authors recommended a multimodal, multisystem approach to pain management in pediatric patients.

Winskill and Andrews (2008) reported the implementation of distraction boxes into nine emergency departments. The distraction box consisted of a range of carefully selected toys geared for children less than 7 years of age, with mild to moderate pain. 
Evaluation of distraction box use and usefulness was conducted 10 months postimplementation by interview over the telephone guided by a five item questionnaire. Of the 13 questionnaires completed the authors reported that all facilities utilized distraction boxes on a regular basis, and staff perceived the distractions to help minimize anxiety and pain experienced by the child. Other comments included the need to remind staff to use the distraction boxes, the difficulty of tracking the box, missing toys, and the variable need for administration of pain medication for children who underwent procedures.

Young, Griffin, Phillips, \& Stanley (2010) piloted a music intervention in the pediatric ED on patients chosen by staff with moderate to severe pain, such as patients experiencing sickle cell crisis, fracture, migraine headache, and those individuals undergoing a painful procedure such as incision and drainage, laceration repair, intravenous line insertion, and lumbar puncture. The staff perceived the music intervention provided distraction from the pediatric patient's pain, but also reduced distress and anxiety in parents. The intervention was reported to improve patient satisfaction as evidenced by increased scores on pain management and overall quality of care.

\section{Communication Related to Comfort in the Emergency Department}

Some researchers have conceptualized that because pain and anxiety are frequently indistinguishable; the combination is referred to as distress (Cohen, Blount, Cohen, \& Johnson, 2004; Jay, 1988; Kennedy \& Luhmann, 1999; Siegel, 1988). In this sense, pediatric pain has been identified to be both physical and emotional; pain and anxiety coexist and potentiate one another (Emergency Nurses Association, 2004). Various 
observational tools developed for pediatric procedural pain incorporate behaviors that measure both anxiety and pain under the concept of distress (Cohen et al., 2004).

In the literature reviewed, an extensive amount has been written on adult communication during painful procedures and child behavioral distress (Blount et al., 1989; Blount, Sturges, \& Power, 1990; Blount et al., 2000; Cline et al., 2006; Cohen et al., 2002; Dahlquist et al., 2001; Fanurik, Koh, \& Schmitz, 2000; Frank, Blount, Smith, Manimala, \& Martin, 1995; Gelfand \& Dahlquist, 2003; Jay, 1988; Manimala, Blount, \& Cohen, 2000; Manne et al., 1992; McCarthy et al., 2010; Naber, Halstead, Broome, \& Rehwaldt, 1995; Slifer et al., 2011; Sweet \& McGrath, 1998; Vessey, Carlson, \& McGill, 1994). Pediatric procedural distress has been a topic of focus mainly in the area of pediatric oncology specific to lumbar punctures, bone marrow aspirations (Blount et al., 1989; Blount et al., 1990; Blount et al., 2000; Cline et al., 2006; Dahlquist et al., 2001; Gelfand \& Dalquist, 2003; Naber et al., 1995) and needle procedures that include venipuncture, immunizations, and intravenous catheter insertion in the inpatient and outpatient setting (Cohen et al., 2002; Fanurik et al., 2000; Frank et al., 1995; Manimala et al., 2000; Manne et al., 1992; McCarthy et al., 2010; Slifer et al., 2011; Sweet \& McGrath, 1998; Vessey et al., 1994).

Research findings on adult communication behavior indicate that certain adult behaviors promote child coping and reduce distress while other behaviors have been shown to increase child distress. Adult behaviors found to promote child coping and minimize distress in children between 5 and 13-years of age during bone marrow aspiration and lumbar puncture included the use of nonprocedural talk, humor to the 
child, and adult coaching of the child to use coping behaviors such as audible deep breathing (Blount et al., 1989; Blount et al., 1990; Blount et al., 2000).

Adult communication behaviors found to increase child distress included reassurance, empathic comments, apologies, criticism, and giving control to the child (Blount et al., 1989; Blount et al., 1990; Blount et al., 2000; Cohen et al., 2002; Frank et al., 1995; Manimala et al., 2000; Sweet \& McGrath, 1998). Manne et al. (1992) examined children 3 to 9-years of age who underwent venipuncture in an outpatient setting and found that the use of praise increased child distress both before and during the procedure, and facilitated child coping during the post procedure phase. During the procedure, distraction and giving the child decisional control (e.g., "Do you want an arm board?") decreased child distress. Adult explanation was found to increase child distress during all phases of a procedure while the use of criticism, threat, bargaining, and commands to cope were associated with higher rates of distress during preparation and completion and increased crying during the procedure. Prior research indicated that giving control to the child by beginning the procedure with saying "Tell me when you are ready," was associated with increased child distress (Blount et al., 1989). Other research that examined adult command structure found that vague commands (e.g., "Get ready") were associated with greater child distress in children 5 to 15 years of age who underwent intramuscular injection and lumbar puncture for cancer procedures (Dahlquist et al., 2001).

Gelfand \& Dahlquist (2003) examined the relationship between child distress and mother and nurse verbal responses in children 2 to 15 -years of age during oncology procedures, the use of general praise was found to not be associated with changes in child 
distress and both maternal and nurses' criticism was significantly related to child distress before, during, and, after the procedure (Gelfand \& Dahlquist, 2003).

Two qualitative studies were located that examined communication patterns during painful pediatric cancer procedures (Cline et al., 2006; Naber et al., 1995). Cline et al. (2006) developed a typology of parent communication, the four communication patterns that emerged: normalizing, invalidating, supportive, and distancing. The most common parent communication pattern was supportive followed by normalizing, and distancing. The patterns used most categorized by procedural phase were normalizing before the procedure, supportive during the procedure, and supportive distancing during the postprocedure with invalidating the least common pattern in all phases; however it was used frequently during the procedural phase. Researchers found that children who were invalidated by the primary parent during the procedure experienced more distress than children of parents who used other communication patterns.

Naber, Halstead, Broome, and Rehwaldt (1995) identified four major communication patterns of behavior labeled talking WITH, talking AT, talking AROUND, and talking TO. Talking with, the child was verbally prompted to use previously taught nonpharmacological techniques. Talking at (e.g., "You're making this harder"), consisted of a combination of mild threats and promises of reward for cooperative behavior. Talking around, was characterized by physician and staff discussion on work related issues when the child was quiet or sedated. Talking to, comprised of social talk among staff and parents about family activities and hobbies. In this study, talking with the child and closeness of the parent was associated with less child distress and talking to the child was associated with increased distress. No studies were located that examined parent or 
healthcare provider communication and child pain and distress during painful pediatric routine procedures in the ED setting.

A review of the child life literature for use in the ED was undertaken because many of the interventions performed by child life specialists (CLS) focus on communication and interaction with parent and child during painful procedures. Some activities provided by CLS include physical comfort, age-appropriate information about events and procedures, communication and education of the family, to calm and reassure patient and family, teach and prompt coping responses. Blount, Bunke, and Zaff (2000) categorized the child coping behaviors of nonprocedural talk and the use of humor by the child as forms of distraction. Child life programs have been used extensively for the pediatric inpatient setting and have been shown to decrease emotional trauma associated with medical procedures encountered during hospitalization.

Only a few studies were located that examined child life services in the ED during routine peripheral venous angiocatheter insertion, venipuncture (Cavender et al., 2004; Stevenson, Bivins, O’Brien, \& Gonzalez del Rey, 2005), suturing (Alcock, Feldman, Goodman, McGrath, \& Park, 1985), environment and waiting behaviors (Alcock, Goodman, Feldman, McGrath, Park, \& Cappelli, 1985), and availability of child life programs in pediatric emergency departments (Krebel, Clayton, and Graham, 1996). Three quantitative studies utilized a multimodal non-pharmacological intervention to assist during painful procedures (Alcock, Feldman et al., 1985; Cavender et al., 2004; Stevenson et al, 2005). The results of these studies documented that child life interventions had no statistical significant effect on children's self-reported pain (Alcock, Feldman et al., 1985; Cavender et al., 2004). This supports previous results from 
quantitative studies that measured self-reported pain and distress of children during intravenous catheter insertion in the inpatient and outpatient setting, findings indicated that the use of behavioral techniques combined with pharmacological intervention were associated with less behavioral distress in children, but there was no significant difference in self-reported pain between the interventional and control groups (Fanurik et al., 2000; McCarthy et al., 2010)

Alcock, Feldman, Goodman, McGrath, \& Park (1985) examined the effects of stressreducing interventions on children who required sutures in the $\mathrm{ED}$, researchers randomly allocated children to a child life intervention group or to a control group. To minimize the possibility of contamination by the environmental presence of the child life program at the pediatric hospital two control groups, control A at the pediatric hospital, and control B at a general hospital were included. Interventions by child life specialist included: provide information, calm, reassure, teach, model, prompt coping responses, to provide physical comfort to parents and children. No significant difference was found between the experimental groups for children's observed anxiety behavior, self-reported anxiety, or self-reported pain. The authors noted that children four to six years of age reported feeling the most pain, scored highest on observed anxiety behavior when six or more sutures were required, and showed more post emergency visit behavior changes than older children. While this is a seminal piece of work in the area of pediatric procedural pain and distress, the results are more than twenty years old.

Cavender, Goff, Hollon, and Guzzetta (2004) examined the effectiveness of parental positioning for comfort and distraction on the level of pain, fear, and distress of pediatric patients between 4 and 11 years who underwent venipuncture or intravenous catheter 
insertion. Children in the comparison group received standard care that included full explanation or the procedure and parental presence for support. Children in the experimental group received standard care in addition to education for parents about how to position and distract the child. Distractions included an illusion kaleidoscope, search and find book or a lift and look book. Of the 43 participants no significant difference was found in children's self-reported pain, self-reported fear, or for behavioral distress as rated by the child life specialist. There was a significant quadratic trend found between the pre-procedural, procedural, and post procedural distress scores that revealed that both the experimental and control group demonstrated the same levels of distress preprocedural, a significant rise in distress during the procedure, and a significant fall post procedure.

Stevenson, Bivins, O’Brien, and Gonzalez del Rey (2005) conducted a randomized controlled trial to examine child procedure-related distress in the ED and the effect of child life interventions during routine peripheral venous angiocatheter insertion on children between 2-16 years of age. The control group received standard care that included explanation of the procedure to the patient and family, and the use of diversional activities for the child and family. The experimental group received pre-procedural support through the establishment of verbal rapport, explanation with the use of visual aids, and response to child and family concerns. Procedural support by the child life specialist consisted of introduction to coping techniques such as visual imagery, auditory distraction, deep breathing exercises, tactile stimulation, counting, singing, verbal interaction, attending to the procedure, parental presence, and praise for successful skills demonstrated by the child. Post procedure support consisted of rewards that included 
stuffed animal, toy, coloring pages, movie to watch, sticker, or Popsicle. Of the 149 subjects who completed the trial, results demonstrated that there was no significant difference between experimental and control groups for parent or child anxiety. There was a statistically significant difference in the intention-to-treat analysis in the anticipatory phase in 4 to 7 -year age group. Explanatory analysis of behavioral distress scores by phase demonstrated the strongest effect in the anticipation and preparation phases of the procedure. The authors concluded that child life interventions may reduce behavioral distress during the anticipation and preparation phase associated with angiocatheter insertion for children 4 to 7-years of age in a pediatric ED setting. Attitudes Related to Pediatric Procedural Pain in the Emergency Department

The empirical literature demonstrates that healthcare provider attitudes and preconceived notions about the patient's age, behaviors, diagnoses and personalities may influence pain management practices (Brockopp, Ryan, \& Warden, 2003; Broome \& Huth, 2003; Hennes et al., 2005; Jones \& Machen, 2003; Kim et al., 2003; Melhuish \& Payne, 2006; Miner, Biros, Trainor, Hubbard, \& Beltram, 2006; Rasmussen, Frederiksen, Hallonsten, \& Poulsen, 2005; Twycross, 2002; Twycross, 2007b; Twycross, 2008; Vincent, 2005; Woodgate \& Kristjanson, 1996a). Studies have demonstrated that numerous assumptions and personal beliefs by healthcare professionals may interfere with the optimal recognition and treatment of pain. In a comparison of pre-hospital providers' perceptions of pain management and practices for adults and children with similar injury, the majority of respondents reported to be uncomfortable working with children (Hennes et al., 2005). Chart review demonstrated that children and adolescents had less documentation of pain assessment and received less analgesic interventions 
when compared with adult patients for the same injury (Hennes et al., 2005). Twycross (2007b) compared observational data to nurses' reported pain management practices and found that no positive relationship was found between nurses' level of theoretical knowledge and how well they actually managed pain.

Different researchers have reported the existence of outdated belief systems such as the ideas that pain during routine procedures are unavoidable and over quickly, children learn to cope with pain as part of life, and the complete absence of pain is a Utopia (Meunier-Sham \& Ryan, 2003; Rasmussen et al., 2005). Various qualitative studies reported similar perceptions of pain management in healthcare providers. Interviews with paramedics reported the belief that some cultures magnify their pain as the patients' feel they are ignored due to language barriers and related issues (Jones \& Machen, 2003). Nurses' have been reported to be guided by value judgments where good children did not complain and children with overt behaviors were described with negative labels for their behavior as hysterical, whining, wimpy, or miserable (Byrne et al., 2001; Woodgate \& Kristjanson, 1996a).

As previously mentioned, nurses' have been found to have a one-sided pharmacological view of pain management where nursing care was synonymous with analgesic administration and that concerns were more practical oriented to cure than to care (Francke, Lemmens, Abu-Saad, \& Grypdonck, 1997). This one-sided idea of pain management has been supported in other studies that demonstrated nurses only assessed pain in patients who were on diamorphine infusions (Twycross, 2002). Woodgate and Kristjanson (1996b) reported nurses did not report comforting children to be their responsibility and that technical aspects of care were more important. They also 
perceived a hierarchy of suffering as a certain amount of pain was expected based on a child's diagnosis and this inadvertently affected how nurses' responded to children's pain, other types of pain such as treatment-related pain were not perceived with the same degree of seriousness (Woodgate \& Kristjanson, 1996a). A lack of concern for procedure related pain in children was also found in a survey of 267 Finnish pediatric nurses' attitudes and beliefs of procedural pain, nurses reported they were prepared to accept some pain in patients during minor procedures and they did not consider it necessary to medicate children before minor procedures (Salanterä, 1999).

The aforementioned findings were limited to nurses' experience on medical, and surgical pediatric units (Melhuish \& Payne, 2006; Salanterä, 1999; Twycross, 2002, 2007b, 2008; Vincent, 2005; Woodgate \& Kristjanson, 1996a), dentists' (Rasmussen et al., 2005), and paramedics in the pre-hospital setting (Hennes et al., 2005; Jones \& Machen, 2003). Of those studies related to emergency practice, findings were based on physicians' perceptions of adult patients in pain (Juhl \& Conners, 2005; Miner et al., 2006), and the opinion and practice of pediatric emergency medicine physicians and pediatric surgeons in children with acute abdominal pain (Kim et al., 2003). Although there is an extensive research base on the knowledge and attitudes of healthcare providers on pain management, few have examined attitudes of ED providers on pediatric procedural pain in young children.

Read (1994) conducted a survey using the Klein Premedication Questionnaire that explored the attitudes and practices of 24 nurses and 21 physicians about children's pain in the emergency setting in a pediatric hospital and community hospital. The questionnaire consisted of three sections, section one gathered information about pain 
assessment, knowledge of analgesics, and perceptions regarding pain management three vignettes were described: vignette one described a 4-year-old girl admitted to the emergency room after being struck by a car while riding her bike femur fracture complaining of pain, vignette two described a 2-year-old boy laceration to the forehead, vignette three 12-year-old girl painful sickle cell crisis. The results revealed that in vignette two the majority of respondents would re-medicate the child if he complained of pain during suturing however $5 \%$ of the physicians and $8 \%$ of the nurses would restrain the child and continue to suture. The majority of physicians and nurses considered pain management appropriate for the children in each scenario; however responses on the pain management items in the questionnaire were incongruent with the actual practice. While this study is rather dated it is considered a seminal work regarding perceptions of nurses and physicians in the emergency department for pain management of pediatric patients.

One study was located that examined healthcare provider perceptions of pain and distress during common procedures in young children in the pediatric ED (Babl et al., 2008). Babl, Mandrawa, O'Sullivan, and Crellin (2008) surveyed medical and nursing staff in an Australian ED to measure staff perceptions of pain and distress experienced by children during fifteen common ED procedures. Of the 92 respondents, results indicated that ED physicians and nurses perceived all ED procedures to be more distressing than painful in young children. The most painful procedure was considered to be suprapubic aspiration (SPA), followed by intramuscular injection (IMI), lumbar puncture (LP), and nasogastric tube insertion (NGT). The procedure considered to cause the most distress was NGT insertion, intravenous insertion (IV), and LP. Both nebulizer and metered dose inhaler medication administration were perceived to cause equal amounts of distress in 
young children. The findings of this study helped to develop an understanding of the hierarchy that ED providers' perceive pain and distress for commonly performed pediatric procedures. Previous results on perceptions of adult pain demonstrated practitioners and patients similarly ranked commonly performed ED procedures; however the use of anesthesia by physicians prior to performing painful procedures was reported to be low despite patient's preference for local anesthetics before similar types of procedures (Singer et al., 1999).

Few instruments were located that measured attitudes regarding pediatric pain management in ED providers; of the two studies located a vignette survey design was employed (Read 1994; Russo, 2010). Manworren (2001) developed and tested the Pediatric Nurses’ Knowledge and Attitudes Survey Regarding Pain (PNKAS) that was a modification of McCaffery and Ferrell's (1997) Nurses' Knowledge and Attitudes Survey Regarding Pain (NKAS). One of the modifications for the PNKAS included the addition of three procedural pain management items dispersed throughout the 42 item survey. The remaining questions tested the subjects' knowledge and attitudes regarding pain assessment, general pain management, and use of analgesics (Manworren, 2001). Manworren (2000) surveyed 274 nurses, which included 36 emergency and transport nurses, at a large children's medical center using the PNKAS. Seventy percent of emergency nurses scored significantly higher than nurses from other patient care units. Of the total participants surveyed, $89.9 \%$ believed that children over reported the amount of pain and over $60 \%$ believed that non drug interventions were rarely helpful for severe pain. 
Various qualitative studies conducted outside the U.S. have found that emergency nurses perceptions of nursing care were viewed as an extension of medicine, where nurses felt more stimulated, technically expert and useful when conducting medical tasks (Byrne \& Heyman, 1997; Nyström, Dahlberg, \& Carlsson, 2003; Nyström, Nydèn et al., 2003). Walsh and Dolan (1999) measured the differences between how emergency nurses viewed care as compared to other nurses. The results demonstrated that ED nurses regarded getting to know the patient and spending time sitting with the patient as significantly less important when compared to the views of other nurses'. These attitudes have also been found with ED physicians' where the mission of the ED was viewed to save lives, concerns about emotional issues were viewed as trivial and interference to the more important work (Hemmelgarn et al., 2001).

\section{Environmental Issues and Policies in the ED}

Position statements and clinical practice recommendations to guide healthcare providers specific to procedural pain, needle-related procedures, (Czarnecki et al., 2011; Emergency Nurses Association, 2010, 2011) and pain assessment of nonverbal patients (Herr et al., 2006; Herr et al., 2011) have been recently published. A review of the literature highlights that despite the implementation of previous regulatory standards and guidelines on pain assessment and management by the American Academy of Pediatrics and the American Pain Society (2001) and the Joint Commission on Accreditation of Healthcare Organizations (2000) the under treatment of procedural pain in children still exits (Bhargava \& Young, 2007; MacLean et al., 2007; Young 2005). Research findings support that the implementation of educational programs and procedural pain management protocols in the ED improved the quality of care (Augarten et al., 2006; 
Meunier-Sham \& Ryan, 2003; Santervas et al., 2010). The successes of such programs were contingent on the treatment of procedural pain as a priority, and the development of multidisciplinary committees with administrative authority to mandate change (MeunierSham \& Ryan, 2003; Schechter, 2008). Contrary to those results some researchers found pain management programs to be less effective for the improvement of procedural pain management and pain documentation (Ellis et al., 2007). Alley (2001) examined the influence of existing organizational pain management policy on nurses' practices and found that knowledge of pain management policy influenced nurses' pain knowledge and perceived accountability, but there was no relationship between nurses' knowledge of policy and treatment of pain. Pasek et al. (2007) implemented a nurse-driven analgesic protocol for children undergoing venipuncture and intravenous cannulation. The researchers found protocol adherence to be low and difficulties to change historic pain practices within the institution.

Healthcare system related barriers have been identified as one of the main contributors to suboptimal pain management in the ED (Duignan \& Dunn, 2008). Researchers have studied the social context of the ED environment and the potential effect on the management of pain with a recent emphasis on effect of ED overcrowding (Bernstein et al., 2009; Duignan \& Dunn, 2009; Hwang, Richardson, Sonuyi, \& Morrison, 2006; Hwang et al., 2008; Pines, Shofer, Isserman, Abbuhl, \& Mills, 2010; Russo, 2010; Tanabe, 1996; Tanabe \& Buschmann, 1999). Of those studies located, three quantitative studies measured ED nurses' perceived barriers to general pain management (Duignan \& Dunn, 2009; Tanabe, 1996; Tanabe \& Buschmann, 1999), four studies examined the effect of ED crowding on quality of pain care, analgesia in patients with 
back pain, the management of pain in older adults with hip fracture, and patients who presented at triage with severe pain (Hwang et al., 2006; Hwang et al., 2008; Pines \& Hollander, 2008; Pines et al., 2010). One qualitative study was located that identified the theme things that hinder decision-making for pediatric pain (Russo, 2010).

Russo (2010) used a phenomenological perspective to examine ED nurse triage decision-making with pediatric pain. Of the themes reported, things that hinder was identified to potentially influence decision making in the treatment of pain for the pediatric patient and was primarily related to environmental issues such as ED crowding, the busy environment, and physicians not giving orders for pain medication when the nurse felt a patient required it.

Tanabe (1996) surveyed ED nurses and asked them to rate what percent of the time each of the barriers listed actually was a barrier to pain management in their practice. Responses included that greater than $25 \%$ of the time the responsibility of caring for other patients, and the lack of time were barriers. Fifty-three percent of the time respondents answered the inability to medicate until a diagnosis was made. High environmental stress was also measured as an independent variable using multiple logistic regressions. The final model indicated that chest pain alone predicted treatment for pain no other variables including environmental stress were significant (Tanabe, 1996).

Duignan and Dunn (2009) conducted a similar survey of emergency nurses and perceived barriers to pain management in the Republic of Ireland, most barriers were described as organizational issues. Respondents rated the frequency of barriers $56 \%$ perceived inability to offer analgesia until a diagnosis was made, $52 \%$ identified a lack of 
time to assess and control pain adequately, $51 \%$ perceived the responsibility of caring for other acutely ill patients in addition to patients with pain, $45 \%$ identified the inability to monitor side effects when patients left the ED for diagnostic procedures, $35 \%$ identified doctors' reluctance to prescribe analgesia, and $23 \%$ perceived overcrowding in the department as barriers to pain management.

Hwang, Richardson, Sonuyi, and Morrison (2006) evaluated the effect of ED crowding on assessment and treatment of pain in older adults with hip fracture. The results demonstrated that census levels greater than $120 \%$ bed capacity was associated with a lower likelihood of documentation of pain assessment, and delays in analgesic administration once pain was identified. The mean time to pain assessment was 40 minutes, time to treatment was 141 minutes, and the mean delay to treatment was 122 minutes.

Pines and Hollander (2008) examined the impact of ED crowding on delays in treatment and non-treatment for patients 18 years and older who presented to triage with severe pain. The authors reported a significant association between measures of ED crowding and delays in treatment and non-treatment with pain medication. Fifty-nine percent of patients waited for at least an hour from triage for medications, $20 \%$ had to wait more than 1 hour from room placement until administration of their first dose of pain medication. These findings are congruent with other studies on ED overcrowding that demonstrated during periods of higher crowding, higher census and high number of boarders (number of admitted patients waiting for inpatient beds) were associated with delays in pain treatment and fewer patients received any form of analgesic medication (Hwang et al., 2008; Pines et al., 2010). Investigation of the extant literature revealed no 
examples of empiric research validating the effect of ED overcrowding on the treatment of pediatric procedural pain. As previously stated, only one study was found which explored the affect of unit culture on nurses' management of children's pain and whether the context in which nursing care was provided contributed to the problem of the under treatment of children's pain (Foster, 1991).

Researchers have studied ED nursing care encounters from a qualitative perspective and found the organization of unit culture played a significant role in the delivery of care (Byrne \& Heyman, 1997; Nyström, Dahlberg et al., 2003; Nyström, Nydèn et al., 2003; Olive, 2003). Byrne and Heyman (1997) identify a key aspect to understanding communication and the delivery of care in the ED was that major traumas, myocardial infarctions, and time sensitive treatments appeared to influence the way in which the unit was organized. Despite the fact that major trauma and patients of that category type formed a small part of the workload the department was geared towards their care. For example, the patients were found to be triaged according to priority in order to seek out the patients in need of urgent care versus the non-urgent patient (Nyström, Dahlberg et al., 2003; Olive, 2003).

Another key aspect of ED care was defined by Byrne and Heyman (1997) as keeping the department running smoothly where activities on the unit were carried out quickly and efficiently so that staff focused on practical tasks, moved patients through the department, and limited the amount of time spent with each patient in order to be prepared and respond to any potential acute medical situations (Nyström, Dahlberg et al., 2003; Nyström, Nydèn et al., 2003; Olive, 2003). Time constraints to deliver treatment; address the needs of the patients waiting, continuous interruptions, the demands from 
multiple physicians at one time, and lack of nursing staff limited nursing care to address physical needs and concrete interventions for the patients (Byrne \& Heyman, 1997; Nyström, Dahlberg et al., 2003; Olive, 2003).

The physical layouts of the department contributed to the fragmentation of the nursepatient encounter, personnel were stationed in specific areas (Olive, 2003). The nursing care of patients was described as a conveyor belt with a high division of labor where the patient was in contact with multiple providers as care was deconstructed and tasks allocated to various nursing professionals and assistants (Nyström, Dahlberg et al., 2003; Nyström, Nydèn et al., 2003; Olive, 2003). The results of these findings taken together demonstrate that the social context appeared to heavily influence nursing care practices.

Research findings on patient satisfaction and pediatric pain have been mixed. Children's satisfaction has been found to be correlated with resolution of pain and that painful procedures made up the worst part of their experience (Chesney, Lindeke, Johnson, Jukkala, \& Lynch, 2005; Magaret, Clark, Warden, Magnusson, \& Hedges, 2002). Parental overall satisfaction has demonstrated different results. Locke, Stefano, Koster, Taylor, and Greenspan (2011) found that caregiver satisfaction, willingness to return and refer others to the ED were related to pain controlled in the child, adequate pain relief, RN attentive to needs and appropriate staff attitudes (Pagmenta \& Benger, 2008); however other studies have found that decreased wait times, and improved work flow were correlated with higher parental satisfaction scores (Chesney et al., 2005; Magaret et al., 2002). Magaret, Clark, Warden, Magnusson and Hedges (2002) found improved parental satisfaction scores related to shorter actual and estimated times spent 
in the waiting room, and shorter actual time spent waiting in the examination room prior to being seen by the doctor.

Pagnamenta and Benger (2008) conducted a prospective questionnaire based study of parents who attended a UK pediatric emergency department to identify factors considered by parents to be most important in determining overall satisfaction with care in their child's emergency department visit. The authors concluded that despite recent emphasis on overall time spent in the ED as a target measurement; parents ranked adequate pain relief for their child, clear explanations, and unrestricted access to their child as the most important factors before wait times and process issues.

Some researchers have further examined parental attitudes and willingness to pay extra money and spend more time in the ED to implement pharmacological interventions for painful procedures. Nelson, Hoagland, and Kunkel (2000) found that of the 81 parents surveyed up to one third of parents did not want to pay extra money or wait extra time for the use of sedation during a hypothetical laceration repair for the child. Eighty-one percent originally stated they would wait 30 extra minutes, but this amount decreased to $73 \%$ if efficacy of the methods was not assured. Parental willingness to pay $\$ 100$ extra was reported to be $75 \%$, but this amount decreased to $67 \%$ if sedation was only effective half the time.

Walsh and Bartfield (2006) found that of the 108 parents, $89 \%$ would consider making their child's hypothetical IV painless, of those parents, $65 \%$ were willing to spend at least 60 extra minutes in the ED, with $93 \%$ willing to stay 20 minutes extra. With regard to cost, $77 \%$ of parents were willing to spend at least $\$ 15$ out of pocket for the painless IV. 


\section{Contribution of Findings to Current Knowledge}

As previously mentioned, this study is unique in that is describes in detail the attitudes and practices of healthcare providers in a non-pediatric emergency department, more specifically, how they assessed and managed pediatric procedural pain within the social context of the unit. No other study has examined pediatric pain in a non-pediatric emergency department from this perspective. This study provides a window into the care of children during painful procedures in the ED setting. The findings of this study provide several contributions to the current literature.

The first contribution of this study is the finding that participants did not measure pain intensity related to common diagnostic or therapeutic procedures of children in the ED. In the literature, recommendations and practice guidelines from professional organizations for the assessment and treatment of pediatric pain include procedural pain; however the majority of studies on pain assessment in the ED are limited to patients who present with identified painful conditions. In this study, participants did not use standardized assessment or measurement tools for procedural pain. Participants' explained that documentation of a child's response was done before and after a procedure, with orthopedic fracture reductions the most common example, stating "you say child tolerated procedure, but with crying or whatever." While participants were aware that a procedure would inflict a certain degree of pain that consideration did not alter the manner care was delivered.

Another important finding is that participants questioned the reliability of pain scales and children's true comprehension of those devices. The measurement of pain intensity appeared to be more reflective of the ED nurses' opinion about how much pain the child 
experienced guided by the participants' perceived hierarchy of suffering based on diagnosis. The hierarchy of suffering was related to a hierarchy of painful procedures, identified as either high end or low end that dictated whether pain was managed pharmacologically or not. The type of painful procedure, length, and invasiveness was perceived by the healthcare provider to determine the degree of pain experienced by the child and need for treatment. These findings support previous results where healthcare providers were more likely to assign higher pain ratings and administer more pain relieving treatments for those patients (Chisholm et al., 2008, Hamers et al., 1994; Woodgate \& Kristjanson, 1996a).

Participants were also observed to rely on overt or active pain behaviors and nonactive behaviors to sensitize them to the presence of a child's pain; however the display of these types of behaviors during painful procedures were not given priority. One of the participants described a situation where a child needed stitches and the mother was concerned that the boy was in pain because he was crying. The participant stated, "He's not in pain, you know when a kid cries in pain, and when a kid just cries. Kids cry no matter what and they need to be held down because the doc can't suture a moving target."

The management of children during painful procedures consisted of an array of behaviors and practices. The use of physical restraint during painful procedures was a common practice and identified by participants as a necessary component of care. Some participants relied on the use of an expert and the rule of efficient delivery, where speed and accuracy were viewed to decrease psychological trauma related to the procedure. The frequency and use of physical restraint during common painful procedures in the ED has not been previously documented in the literature. 
A typology of communication behaviors used by participants related to comfort is presented. The most frequently used communication behaviors during a procedure were: reassurance, positive reinforcement, parental presence, and positioning, with reassurance the most common behavior. Participant behaviors prior to a procedure included: procedural preparatory information, sensory preparatory information, and child directed speech. Deviance from communication related to comfort criticism, condescension, and dismissive remark is also presented.

An interesting finding is that participants describe the uneasiness of staff due to the lack of practice in taking care of and performing procedures on young children. An activity observed and described as passing the buck is presented. Passing the buck was described by one participant as when a healthcare provider took an activity they did not want to do or did not feel capable and handed the activity to a different person either on their level or below their level that was capable of doing the task. The concept of passing the buck was understood in this study to be a form of negative delegation. No studies were located that specifically examined the uneasiness of ED staff in a non-pediatric ED and the care of young children. Previous studies were located that discussed negative delegation practices between registered nurses and assistive personnel on medicalsurgical and oncology units (Kalisch, 2006; Potter, Deshields, \& Kuhrik, 2010), but none specific to the ED setting.

Many assumptions are still held by providers related to the consequences of untreated procedural pain. While there was a general consensus among participants that painful procedures were stressful and traumatic for children to undergo in the ED, 
participants varied in their responses as to the potential long term consequences from such brief encounters.

In this study, organizational policies and environmental issues did create conditions that played a significant role in the under treatment of pediatric pain. The interplay and balance of ED demands, organizational values, and accreditation standards appeared to heavily influence participants pediatric pain management practices. For example lack of a pre-medication policy for common needle-related procedures, ED overcrowding, prioritization of high acuity patients and time sensitize treatments were observed to be the greatest barriers to optimal pediatric procedural pain management. Nurse availability and verbal communication of a patient's pain from one healthcare provider to another affected the time line of delivery from assessment to prescription and administration of analgesic.

\section{Summary}

In this chapter research assumptions were revisited and discussed, the findings of the study were compared to what was known about pediatric pain in the emergency department setting and the contributions of this research to the current literature were presented. 


\section{Chapter VII}

\section{Conclusion}

The results of this study suggest that the practice environment of the ED unit and organizational culture exerted a significant degree of influence over healthcare providers' pediatric pain management practices. A majority of the participants believed they adequately managed children's pain. While it appears that there has been some improvement toward the treatment of pediatric pain with the use of topical anesthetics prior to sutures, there is still a need to improve pain management practices for the more common needle procedures. In this study, participants placed a high degree of priority to the treatment of the sensory aspects of pain especially for children who required sutures. In contrast, no standardized approach was used to address the situational, cognitive, behavioral, and emotional factors that intensified a child's perception of procedural pain. Different communication behaviors related to a child's comfort were observed; however, the fact that there was a room filled with bears intended to be used as rewards or comfort measures, and was rarely given out highlights that non-pharmacological techniques were provided when time allowed and viewed by participants to be more of a trivial aspect to care versus a priority. In this study, deviations from communication related to comfort; dismissive remarks, condescension, and criticism, were behaviors observed to occur with only male participants. This interpretation of gender bias must be interpreted cautiously as the sample of men in this study that includes physicians, mid-levels, nurses, and technicians was extremely small.

Nurses' did not use pain measurement tools as they were intended to be used and instead improvised measurement by observing the child's behavior. Participants held 
many assumptions and misconceptions about pediatric pain assessment, management, and the potential adverse effects related to the under treatment of pain. The findings are consistent with attitudinal studies that documented the persistence of misconceptions about children's pain and the need for aggressive management.

In this study there was no racial disparity observed with regard to the treatment of children and their pain; however, participants tended to shy away and deliver less psychological support to children whose parents were described to hover over their children. Patients of Middle Eastern Asian descent were inferred by some participants as a group who generally asked a barrage of questions and hovered over their children. As mentioned previously, over $80 \%$ of the population in the area was white and $3 \%$ of Asian descent. While this finding was noted in only a few participants' remarks, additional research in this area is needed to explore how nurses perceive and manage children's pain based on patient ethnicity.

Certain participants developed an uneasiness to work with and perform invasive procedures on children due to the low volumes of those types of patients to the ED setting. The delegation of those tasks to self-identified pediatric experts in the department was a practice frequently utilized that contributed to the lack of skill development for those participants who were uncomfortable with pediatric patients. This finding that suggested the uneasiness of ED healthcare providers working with children has not been previously documented in the literature.

The practice of physical restraint for children during painful procedures caused an increase in child distress. The interpretation of overt active pain behaviors such as pulling away from the needle injection, screams, and crying did not cause participants to stop and 
re-evaluate for the presence of pain or how a child felt. In addition, one of the most common techniques used to comfort a child was reassurance which has been identified in previous studies to cause an increase in child distress.

No formal documentation of treatment for procedural pain or distress was noted on a patient's medical record unless a pharmacological intervention was administered. The use of physical restraint during a procedure, the number of people involved, and the use of non-pharmacological treatments such as distraction were not included on the patient's chart. The findings of this study provide insight to the everyday practices of ED healthcare providers for pediatric pain. Several findings have been put forward, all of which need further exploration.

\section{Strengths and Limitations}

The first strength of this study is that multiple sources of data served as different sources for confirmation and corroboration. For example, information elicited from observation or interview with key informants was corroborated by information from other participants. Data collected from organizational and unit policies were cross checked by field observations or interviews with participants. Hospital organizational concerns and issues were cross checked with local and statewide media sources.

The second strength of this study is that the researcher was immersed in the nonpediatric ED unit for a prolonged period of 5 months and gathered data through participation and interaction in the daily life of the unit. The researcher was an instrument for data collection that allowed and exposed the researcher to aspects of pediatric procedural pain practice activities that were not normally part of formal instruments that measured pain, anxiety, or fear during procedures. For example, the use of physical 
restraint and holding down the child was a practice commonly overlooked by participants during painful procedures but highlighted several times in the data.

The third strength of this study is that pediatric pain management practices of healthcare providers have been systematically examined in a holistic manner where behavior and events are studied in a cultural context. Events are studied as they evolved in the natural setting representative of ongoing reality without the researcher controlling the process. The relationship of events, behaviors, and practices are further examined within the broader sociocultural milieu of the ED unit and hospital organization.

The final strength of this study is the detailed and comprehensive descriptions of healthcare provider behaviors during pediatric painful procedures and the account of their attitudes and values within those experiences. The data describe a range of attitudes and strategies used by physicians, nurse practitioners, physician assistants, and assistive personnel during the assessment and management of children in pain.

There are several limitations in this study. The first limitation of this study is that data are representative of the practices of one ED unit in one geographic region of the U.S. in a suburban region and as such may not be generalized to all non-pediatric ED's in all areas of the country. The practice of healthcare providers surrounding procedures reflects that of a general community emergency room center and may not be generalized to hospitals with pediatric specialty staff. However, the observed practices included a varied case mix of providers and patients from a full range of social and cultural backgrounds.

The second limitation of this study is that of the sample of key informants and participants, not all healthcare providers who worked in the ED unit during the study 
period consented to participate. The sample only contained those ED providers motivated enough to allow the researcher to observe their practice. Participation and selection of participants was confined mainly to the time of the prompt care hours 9 a.m. to- 1 a.m., therefore representativeness of the data is limited as the population represents a select group of providers and the patients who presented to the ED during those time frames.

The third limitation of this study is that participants were not blinded to the purpose of the observation and thus may have influenced their responses and practices in the assessment and treatment of pediatric pain. During this study, Fellowship hospital was in the process of expanding their pediatric service line to the community, and the presence of the researcher was perceived by ED providers as an evaluation of their competence and capability for providing pediatric care. The fact that participants signed consent forms that explained the purposes of the study had little impact to assuage their concerns. For example, one of the participants stated:

...it's been talked about we've been going through the process about how the main hospital is about peds, and it's no surprise that we go peds and low and behold you come along, so we're all wondering why you're here... and I don't know if it's to see if we are competent, capable, and ready to take on such a task... (Graham)

The final limitation of this study is that of researcher bias. The researcher as an instrument for data collection, interpretation of findings, and descriptions of findings is subject to some degree of bias. Bias in observation may have included overlooked or missed behaviors, sensitization and de-sensitization to practices over time.

\section{Implications for Knowledge Generation and Practice}

The findings of this study provide a picture of how providers in this ED setting assess and manage pediatric pain. Understanding healthcare provider views, and the manner that 
care is delivered may be useful to those professionals in the organization planning to design and implement change for improved pediatric pain management practices. The findings point to the need for pediatric procedural pain to be considered a priority in order to develop initiatives, educational programs, and policies geared to addressing those issues. An implication of this study is that hospitals and organizational leaders should foster an environment and develop a culture that values the treatment of pediatric procedural pain to ensure optimal care.

This study reinforces the disparity between the availability of interventions for pediatric procedural pain and the actual practices of those interventions in the ED setting. Participants in this research study felt constrained by lack of physician prescription, and lack of institutional policy for addressing painful needle procedures. Participants were not observed to routinely use any pharmacological pain treatments for minor procedures such as intravenous line placements, venipunctures, or intramuscular injections.

Participants were found in this study to improvise the use of pediatric pain scales and rely on observation of a child's behavior. It is important to keep in mind that the literature documents pain ratings by nurses and children to be significantly different and that if possible a child's self-report of pain should be elicited. Healthcare providers should gain insight from this finding and incorporate the use of FLACC scales for those children unable to rate pain intensity rather then to assign an arbitrary numerical or FACE score on the medical record. Future research should examine the meaningfulness of pain as a $5^{\text {th }}$ vital sign and whether routine documentation of pain in children improves the quality of pain management treatment. 
From a nursing perspective, the concern should be on the best way to approach and integrate strategies to relieve pain from procedures in the ED setting for young children. The finding regarding the frequent use of physical restraint on children during painful procedures should sensitize nurses to further study this phenomenon. Future research is needed to address if the findings from this study are reflective of current practice in other ED settings. It would be recommended to compare the pediatric pain practices of providers in pediatric specific emergency departments to explore whether environmental influences of the ED setting such as overcrowding, keeping the flow, streamlining of patients, and time management preparing for uncertainty influence the implementation of pain management practices.

The results of this study provide an opportunity to formulate new questions for further research, such as: what are emergency nurses attitudes toward pediatric procedural pain for common needle procedures?; what are the current practices for procedural pain in pediatric emergency departments?; how does ED overcrowding effect the treatment of pediatric procedural pain?; does the existence of a pain policy for the pre-medication of children prior to needle procedures decrease child pain or distress?; what are the practices of nurses for the use of restraint and immobilization on pediatric patients during painful procedures in the ED?

\section{Recommendations}

Based on the findings of this study, further research on pediatric procedural pain in the non-pediatric ED is recommended. Healthcare providers should recognize and acknowledge that unit and organizational culture heavily influence pain management practices. Researchers should consider using Schein's (1992) conceptual model of 
organizational culture and leadership to study organizational phenomena such as suboptimal pediatric pain treatment practices by providers. Schein (1992) adopted an evolutionary perspective where cultures begin with leaders who impose their values and assumptions on a group, and when those values and assumptions are accepted and incorporated into the fabric of how individuals perceive, think, and feel in relation to issues, culture is then defined.

Healthcare providers should form a multidisciplinary team with administrative authority to mandate change for pain management practices on the ED unit and for the organization. A policy should be developed with the objective of guiding practice for premedication prior to minor needle procedures in young pediatric patients. The American Society for Pain Management Nursing (ASPMN) recommended that nurses collaborate with other members of the health care team to establish policies and procedures outlining the expectations for procedural comfort management before, during, and after painful procedures (Czarnecki et al., 2011).

Pain has been identified as both sensory and emotional therefore healthcare providers should manage children's pain addressing both aspects. The literature documents that non-pharmacological interventions have been shown to decrease distress, but have little to no effect on children's self-report of pain. The need to incorporate a pharmacological approach with a non-pharmacological approach is a necessary component of managing pediatric patients during painful procedures in the ED, provided with this evidence, the goal of treatment for pediatric pain should not be an "ouchless experience" that addresses only one aspect of pain, but to provide the most comfort possible. Healthcare providers should strive for pediatric comfort during painful procedures and atraumatic care. The 
concept of atraumatic care has been defined as the provision of therapeutic care in such a way as to minimize the physical and emotional distress to children and families (Ellis, Sharp, Newhook, \& Cohen, 2004).

While caring for children during procedures, providers should be aware of common distress promoting behaviors such as reassurance, a communication behavior frequently used by participants in this study. Providers should incorporate and promote more child coping strategies to manage pain and distress during painful procedures in the ED. Nursing education should develop a curriculum to train providers as to the best strategies for managing pediatric distress, and the most effective way to deliver those strategies or interventions within the environment of the ED.

Physical restraint was a frequent practice used by participants in this study; however, there is a lack of empirical research on the use of those practices in the ED setting. In this study, it was observed that nurses and assistive personnel were responsible for the implementation of this task.

It is a recommendation that providers who participate in those activities be formally trained. Curriculum development should include ethical considerations, legal responsibility, local laws and professional organization practice statements that address restraint, applicable de-escalation techniques, safe methods of how to appropriately hold and restrain children, and documentation requirements (Mohr et al., 2003). Further research is needed to address the effect of restraint use on children during painful procedures in the ED and their families.

A concern frequently cited in this study and discussed in the literature involved the validity of pain scales for use in the ED setting. Two behavioral pain and distress scales 
have been recommended, the CHEOPS and FLACC scales, as measures of procedural pain for clinical practice and research in the ED (Crellin et al., 2007). Further validation studies for the use of these tools for preverbal and early verbal children undergoing painful procedures in the ED is required.

A new finding from this study is the uneasiness of healthcare providers performing procedures on young children. It would be ideal for all ED nurses to gain the appropriate comfort and skill necessary to manage children in the ED; however, economic constraints may not make that feasible. Grant and Crouch (2011) reviewed contemporary issues around who should deliver emergency care to children in the ED settings. The authors highlighted how children were mainly cared for in the UK by adult registered nurses due to the insufficient numbers of registered children's nurses, emergency departments functioned with the minimal level of one registered children's nurse per shift. The authors developed a competency framework for adult ED nurses that included eight core categories: physiological and psychological development, taking and interpreting vital signs, pain management, medicine management, management of the sick child, management of the injured child, mental health, and safeguarding children and young people. This framework mirrors similar topics offered by the Emergency Nursing Pediatric Course offered by the Emergency Nurses Association in the US. While both curriculums appear to be rich in content, it does little to develop the needed hands-on technical skills for working with younger children.

Emergency department leadership needs to invest in the development of nurses to work with young children who present to the department. It is a recommendation of this study that staff skill mix, at the minimum, should include that at least two nurse and assistive 
nursing personnel trained and competent for performing procedures on young pediatric patients. Nurse educators should consider the use of patient simulators to provide ED nursing staff with hands on experience in a safe environment to develop their skills and comfort for working with young children.

\section{Summary}

In this study, it is described that a major source of influence on the practices of ED personnel related to pediatric procedural pain management may have been explained in the characteristics of the unit and the environment of the organization rather than individual providers' characteristics. Previous researchers have demonstrated that environmental culture has played a significant role that perpetuated attitudes and behaviors among employees that either complemented or inhibited the implementation of family centered care, shared views and practices were more a function of the organization in which providers worked than of their own profession, experiences, or gender (Hemmelgarn et al., 2001).

Although extensive research and policy has been developed to address pediatric procedural pain, a gap still exits between what is known about child pain and distress and what is implemented in practice. This study examined the pediatric pain management practices and attitudes of healthcare providers in a non-pediatric emergency department. Through the use of ethnography, a view into the modern day environment of the emergency department and pain management practices were highlighted. This study described the everyday practices of healthcare providers and their management of pediatric procedural pain against the background of the ED environment, organizational policy and national standards. 


\section{References}

Aamodt, A.M. (1982). Examining ethnography for nurse researchers. Western Journal of Nursing Research, 4(2), 209-221.

Aamodt, A.M. (1991). Ethnography and epistemology: Generating nursing knowledge. In J.M. Morse (Ed.), Qualitative nursing research: A contemporary dialogue (Rev ed., pp.40-53). Newbury Park: Sage Publications.

Abbe, M., Sudano, J.J., Demko, C.A., Victoroff, K.Z., Williams, K., Lalumandier, J.A., \& Wotman, S. (2007). Revisiting comfort: Strategies observed in the direct observation study. General Dentistry, 55, 420-425.

Abu-Saad, H. (1984). Cultural components of pain: The Arab-American child. Issues in Comprehensive Pediatric Nursing, 7, 91-99.

Adriansson, C., Suserud, B.O., \& Bergbom, I. (2004). The use of topical anesthesia at children's minor lacerations: An experimental study. Accident and Emergency Nursing, 12, 74-84.

Agency for Health Care Policy \& Research (AHCPR). (1992). Acute pain management: Operative or medical procedures and trauma. Clinical practice guidelines. (Publication No. 92-0032 ed.). Rockville, MD: AHCPR.

Alcock, D.S., Feldman, W., Goodman, J.T., McGrath, P.J., \& Park, J.M. (1985). Evaluation of child life intervention in emergency department suturing. Pediatric Emergency Care, 1(3), 111-115.

Alcock, D., Goodman, J., Feldman, W., McGrath, P.J., Park, M., \& Cappelli, M. (1985). Environment and waiting behaviors in emergency waiting areas. $C H C, 13(4), 174-$ 180. 
Alexander, J., \& Manno, M. (2003). Underuse of analgesia in very young pediatric patients with isolated painful injuries. Annals of Emergency Medicine, 41(5), 617622.

Allcock, N., McGarry, J., \& Elkan, R. (2002). Management of pain in older people within the nursing home: A preliminary study. Health and Social Care in the Community, $10(6), 464-471$.

Alley, L.G. (2001). The influence of an organizational pain management policy on nurses' pain management practices. Oncology Nursing Forum, 28(5), 867-874.

American Academy of Pediatrics. (1997). The use of physical restraint interventions for children and adolescents in the acute care setting. Pediatrics, 99(3), 497-498.

American Academy of Pediatrics, Committee on Pediatric Emergency Medicine. (2007). Patient safety in the pediatric emergency care setting. Pediatrics, 120(6), 1367-1375. American Academy of Pediatrics \& American Pain Society. (2001). Policy statement: The assessment and management of acute pain in infants, children, and adolescents. Pediatrics, 108(3), 793-797.

Augarten, A., Zaslansky, R., Pharm, I.M., Minuskin, T., Lerner-Geva, L., HirshYechezkel, G., Ziv, A., Shavit, I., Yativ, N., \& Keidan, I. (2006). The impact of educational intervention programs on pain management in a pediatric emergency department. Biomedicine \&Pharmacotherapy, 60, 299-302.

Babl, F.E., Mandrawa, C., O’Sullivan, R., \& Crellin, D. (2008). Procedural pain and distress in young children as perceived by medical and nursing staff. Pediatric Anesthesia, 18, 412-419.

Baxter, J. (1993). Stewart smiled. Pediatrics, 91(5), 1018. 
Belville, R.G., \& Seupaul, R.A. (2005). Pain measurement in pediatric emergency care: A review of the Faces pain scale-revised. Pediatric Emergency Care, 21(2), 90-93. Benbenbishty, J., Adams, S. \& Endacott, R. (2010). Physical restraint use in intensive care units across Europe: The PRICE study. Intensive \& Critical Care Nursing, $26(5), 241-245$.

Bernstein, S.L., Aronsky, D., Duseja, R., Epstein, S., Handel, D., Hwang, U., McCarthy, M., John McConnell, K., Pines, J.M., Rathlev, N., Schafermeyer, R., Zwemer, F., Schull, M., Asplin, B.R., Society for Academic Emergency Medicine, \& Emergency Crowding Task Force. (2009). The effect of emergency department crowding on clinically oriented outcomes. Academic Emergency Medicine, 16(1), 1-10.

Besenski, L.J., Forsyth, S.J., \& von Baeyer, C.L. (2007). Screening young children for their ability to use self-report pain scales. Pediatric Pain Letter, 9(1), 1-7.

Bhargava, R., \& Young, K.D. (2007). Procedural pain management patterns in academic pediatric emergency departments. Academic Emergency Medicine, 14(5), 479-482.

Bishop-Kurylo, D. (2002). Pediatric pain management in the emergency department. Topics in Emergency Medicine, 24(1), 19-30.

Bland, M., Bridge, C., Cooper, M., Dixon, D., Hay, L., \& Zerbato, A. (2002). Procedural restraint in children's nursing: Using clinical benchmarks. Professional Nurse, 17(12), 712-715.

Blount, R.L., Corbin, S.M., Sturges, J.W., Wolfe, V.V., Prater, J.M., \& James, L.D. (1989). The relationship between adults' behavior and child coping and distress during BMA/ LP procedures: A sequential analysis. Behavior Therapy, 20, 585-601. Blount, R.L., Sturges, J.W., \& Powers, S.W. (1990). Analysis of child and adult behavior 
variations by phase of medical procedures. Behavior Therapy, 21, 33-48.

Blount, R.L., Bunke, V.L., \& Zaff, J.F. (2000). The integration of basic research, treatment research, and clinical practice in pediatric psychology. In D. Drotar (Ed.) Handbook of Research in Pediatric and Clinical Child Psychology (pp. 491-510). New York: Kluwer Academic/ Plenum Publishers.

Blount, R.L., Piira, T., \& Cohen, L.L. (2003). Management of pediatric pain and distress due to medical procedures. In M.C. Roberts (Ed.), Handbook of pediatric psychology ( $3^{\text {rd }}$ ed., pp.216-233). New York: The Guilford Press.

Brenner, M. (2007a). Restraint in children's nursing: Addressing the distress. Journal of Children's and Young People's Nursing, 1(4), 159-162.

Brenner, M. (2007b). Child restraint in the acute setting of pediatric nursing: An extraordinarily stressful event. Issues in Comprehensive Pediatric Nursing, 30, 2937.

Brewer, J.D. (2000). Ethnography. Philadelphia, PA: Open University Press.

Brockopp, D.Y., Ryan, P., \& Warden, S. (2003). Nurses' willingess to manage the pain of specific groups of patients. British Journal of Nursing, 12 (7), 409-415.

Broome, M.E., Richtsmeier, A., Maikler, V., \& Alexander, M. (1996). Pediatric pain practices: A national survey of health professionals. Journal of Pain and Symptom Management, 11 (5), 312-320.

Broome, M.E., \& Huth, M.M. (2003). Nursing interventions for pain control in children. In N.L. Schechter, C.B. Berde, \& M. Yaster (Eds.), Pain in infants, children, \& adolescents ( $2^{\text {nd }}$ ed., pp.417-433). Philadelphia: Lippincott Williams \& Wilkins.

Brown, J. (1992). Nurse or technicians? The impact of technology on oncology nursing. 
Canadian Oncology Nursing Journal, 2(1), 12-17.

Brown, J. (2002). Registered nurses' choices regarding the use of intradermal lidocaine for intravenous insertions: The challenge of changing practice. Pain Management Nursing, 3(2), 71-76.

Brown, J.C., Klein, E.J., Lewis, C.W., Johnston, B.D., \& Cummings, P. (2003).

Emergency department analgesia for fracture pain. Annals of Emergency Medicine, 42(2), 197-205.

Bulloch, B., \& Tenenbein, M. (2002). Validation of 2 pain scales for use in the pediatric emergency department. Pediatrics, 110(3), e33-e38.

Byrne, G., \& Heyman, R. (1997). Understanding nurses' communication with patients in accident and emergency departments using a symbolic interactionist perspective. Journal of Advanced Nursing, 26, 93-100.

Byrne, A., Morton, J., \& Salmon, P. (2001). Defending against patients’ pain: A qualitative analysis of nurses' responses to children's postoperative pain. Journal of Psychosomatic Research, 50, 69-76.

Carlson, K.L., Broome, M., \& Vessey, J.A. (2000). Using distraction to reduce reported pain, fear, and behavioral distress in children and adolescents: A multisite study. JSPN, 5(2), 75-85.

Carter, L.C., Nelson, J.L., Sievers, B.A., Dukek, S.L., Pipe, T.B., \& Holland, D.E. (2008). Exploring a culture of caring. Nursing Adminstration Quarterly, 32(1), 57 63.

Cavender, K., Goff, M.D., Hollon, E.C., \& Guzzetta, C.E. (2004). Parents’ positioning and distracting children during venipuncture: Effects on children's pain, fear, and 
distress. Journal of Holistic Nursing, 22 (1), 32-56.

Chambliss, D.F. (1996). Beyond caring hospitals, nurses, and the social organization of ethics. Chicago: The University of Chicago Press.

Chesney, M., Lindeke, L., Johnson, L., Jukkala, A., \& Lynch, S. (2005). Comparison of child and parent satisfaction ratings of ambulatory pediatric subspecialty care. Journal of Pediatric Health Care, 19(4), 221-229.

Chisholm, C.D., Weaver, C.S., Whenmouth, L.F., Giles, B., \& Brizendine, E.J. (2008). A comparison of observed versus documented physician assessment and treatment of pain: The physician record does not reflect the reality. Annals of Emergency Medicine, 52(4), 383-389.

Cimpello, L.B., Khine, H., \& Avner, J.R. (2004). Practice patterns of pediatric versus general emergency physicians for pain management of fractures in pediatric patients. Pediatric Emergency Care, 20(4), 228-232.

Cline, R.J.W., Harper, F.W.K., Penner, L.A., Peterson, A.M., Taub, J.W., \& Albrecht, T.L. (2006). Parent communication and child pain and distress during painful pediatric cancer treatments. Social Science \& Medicine, 63, 883-898.

Cohen, L.L. (2002). Reducing infant immunization distress through distraction. Health Psychology, 21, 207-211.

Cohen, L.L., Bernard, R.S., Greco, L.A., \& McClellan, C.B. (2002). A child-focused intervention for coping with procedural pain: Are parent and nurse coaches necessary? Journal of Pediatric Psychology, 27(8), 749-757.

Cohen, L.L., Blount, R.L., Cohen, R.J., \& Johnson, C. (2004). Dimensions of pediatric procedural distress: Children's anxiety and pain during immunizations. Journal of 
Clinical Psychology in Medical Settings, 11(1), 41-47.

Cohen, L.L., Lemanek, K., Blount, R.L., Dahlquist, L.M., Lim, C.S., Palermo, T.M., McKenna, K.D., \& Weiss, K.E. (2007). Evidence-based assessment of pediatric pain. Journal of Pediatric Psychology, pp. 1-17. doi:10.1093/jpepsy/jsm103

Cohen, M.Z., Easley, M., Ellis, C., Hughes, B., Ownby, K., Rashad, B.G., Rude, M., Taft, E.,\& Westbrooks, J.B. (2003). Cancer pain management and the JCAHO's pain standards an institutional challenge. Journal of Pain and Symptom Management, $25(6), 519-527$.

Collins, P. (1999). Restraining children for painful procedures. Paediatric Nursing, 11(3), 14-16.

Comley, A.L., \& Banks, C. (2000). Pain management: Clinician survey and institutional needs assessment. Baylor University Medical Center Proceedings (BUMC), 13, 230235.

Cordell, W.H., Keene, K.K., Giles, B.K., Jones, J.B., Jones, J.H., \& Brizendine, E.J. (2002). The high prevalence of pain in emergency medical care. The American Journal of Emergency Medicine, 20, 165-169.

Craig, K., Lilley, C., \& Gilbert, C. (1996). Barriers to optimal pain management in infants, children, and adolescents social barriers to optimal pain management in infants and children. The Clinical Journal of Pain, 12(3), 232-242.

Craig, K.D., \& Pillai Riddell, R.R. (2003). Social influences, culture, and ethnicity. In P.J. McGrath and G.A. Finley (Eds.), Pediatric pain: Biological and social context: Progress in pain research and management Volume 26 (pp.159-182). Seattle: IASP Press. 
Cregin, R., Rappaport, A.S., Montagnino, G., Sabogal, G., Moreau, H., \& Abularrage, J.L. (2008). Improving pain management for pediatric patients undergoing nonurgent painful procedures. American Journal of Health-System Pharmacy, 65, 723-727.

Crellin, D., Sullivan, T.P., Babl, F.E., O’Sullivan, R., \& Hutchinson, A. (2007). Analysis of the validation of existing behavioral pain and distress scales for use in the procedural setting. Pediatric Anesthesia, 17, 720-733.

Crocker, P.J., Higginbotham, E., King, B.T., Taylor, D., \& Milling, T.J. (2011). Comprehensive pain management protocol reduces children's memory of pain at discharge from the pediatric ED. American Journal of Emergency Medicine, (in press corrected proof) Retrived from: http://O-

$\underline{\text { www.sciencedirect.com.aupac.lib.athabascau.ca/science/article/pii/s0735675711002695 }}$

Crowley, M.A., Storer, A., Heaton, K., Naccarato, M.K., Proehl, J.A., Moretz, J.D., \& Li, S. (2011). Emergency nursing resource: Needle-related procedural pain in pediatric patients in the emergency department. Journal of Emergency Nursing, 37(3), 246251.

Cummings, E.A., Reid, G.J., Finley, G.A., McGrath, P.J., \& Ritchie, J.A. (1996). Prevalence and source of pain in pediatric inpatients. Pain, 68, 25-31.

Czarnecki, M.L., Turner, H.N., Collins, P.M., Doellman, D., Wrona, S., \& Reynolds, J. (2011). Procedural pain management: A position statement with clinical practice recommendations. Pain Management Nursing, 12(2), 95-111.

Dahlquist, L.M., Pendley, J.S., Power, T.G., Landthrip, D.S., Jones, C.L., \& Steuber, C.P. (2001). Adult command structure and children's distress during the anticipatory phase of invasive cancer procedures. Children's Health Care, 30(2), 151-167. 
Dalton, J., Carlson, J., Blau, W., Lindley, C., Greer, S.M., \& Youngblood, R. (2001). Documentation of pain assessment and treatment: How are we doing? Pain Management Nursing, 2(2), 54-64.

D’Arcy, Y.M. (2007). Pain management: Evidence-based tools and techniques for nursing professionals. Marblehead, MA: HCPro, Inc.

Demir, A. (2007a). Nurses' use of physical restraints in four Turkish hospitals. Journal of Nursing Scholarship, 39(1), 38-45.

Demir, A. (2007b). The use of physical restraints on children: Practices and attitudes of paediatric nurses in Turkey. International Nursing Review, 54, 367-374.

Denzin, N.K., \& Lincoln, Y.S. (2003). Strategies of qualitative inquiry (2 ${ }^{\text {nd }}$ ed.). Thousand Oaks: Sage Publications.

Dorfman, D.H., \& Kastner, B. (2004). The use of restraint for pediatric psychiatric patients in the emergency departments. Pediatric Emergency Care, 20(3), 151-156.

Dorfman, D.H., \& Mehta, S.D. (2006). Restraint use for psychiatric patients in the pediatric emergency department. Pediatric Emergency Care, 22(1), 7-12.

Drendel, A.L., Brousseau, D.C., \& Gorelick, M.H. (2006). Pain assessment for pediatric patients in the emergency department. Pediatrics, 117(5), 1511-1518.

Duignan, M., \& Dunn, V. (2008). Barriers to pain management in emergency departments. Emergency Nurse, 15(9), 30-34.

Duignan, M., \& Dunn, V. (2009). Perceived barriers to pain management. Emergency Nurse, 16(9), 31-35.

Ellis, J.A., Sharp, D., Newhook, K., \& Cohen, J. (2004). Selling comfort: A survey of interventions for needle procedures in a pediatric hospital. Pain Management 
Nursing, 5(4), 144-152.

Ellis, J.A., McCleary, L., Blouin, R., Dube, K., Rowley, B., MacNeil, M., \& Cooke, C. (2007). Implementing best practice pain management in a pediatric hospital. JSPN, 12(4), 264-277.

Ely, B. (2001). Pediatric nurses' pain management practice: Barriers to change. Pediatric Nursing, 27(5), 473-480.

Emergency Nurses Association. (2004). Procedural preparation and sedation. In Emergency Nurses Association, Emergency nursing pediatric course provider manual ( $3^{\text {rd }}$ ed., pp. 223-235). Des Plaines, IL: Emergency Nurses Association. Emergency Nurses Association. (2010). Position statement: Pediatric procedural pain management. Retrieved from http://www.ena.org/SiteCollectionDocuments/PositionStatements/PediatricProceduralPai nManagement.pdf

Emergency Nurses Association. (2011). ANA recognizes emergency nursing as specialty practice. Retrieved from https://www.ena.org/media/PressReleases/Pages/ANARecognizes.aspx

Emerson, R.M., Fretz, R. I., \& Shaw, L.L. (1995). Wrtiting ethnographic fieldnotes. Chicago: The University of Chicago Press.

Fanurik, D., Koh, J.L., \& Schmitz, M.L. (2000). Distration techniques combined with EMLA: Effects on IV insertion pain and distress in children. Children's Health Care, 29(2), 87-101.

Fetterman, D.M. (1998). Ethnography step by step $\left(2^{\text {nd }}\right.$ ed.): Applied social research methods series volume 17. Thousand Oaks: Sage Publications. 
Fisher, B.J., \& Peterson, C. (1993). She won't be dancing much anyway: A study of surgeons, surgical nurses, and elderly patients. Qualitative Health Research, 3(2), $165-183$.

Foster, R.L. (1991). The effect of unit culture on nurses' management of children's pain. Journal of Pain and Symptom Management, 6(3), 202.

Francke, A.L., Lemmens, A.B.T., Abu-Saad, H.H., \& Grypdonck, M. (1997). Nurses' perceptions of factors influencing the use of a pain program. Journal of Pain and Symptom Management, 14(5), 300-310.

Frank, N.C., Blount, R.L., Smith, A.J., Manimala, M.R., \& Martin, J.K. (1995). Parent and staff behavior, previous child medical experience, and maternal anxiety as they relate to child procedural distress and coping. Journal of Pediatric Psychology, 20(3), 277-289.

Fry, M., Holdgate, A., Baird, L., Silk, J., \& Ahern, M. (1999). An emergency department's analysis of pain management patterns. AENJ, 2(1), 31-36.

Garg, S., Narsinghani, U., Bhutta, A.T., Rovnaghi, C.R., \& Anand, K.J.S. (2003). Longterm effects of neonatal pain: The animal literature. In P.J. McGrath, and G.A. Finley (Eds.), Pediatric pain: Biological and social context: Progress in pain research and management Volume 26 (pp. 1-22). Seattle: IASP Press.

Garra, G., Singer, A.J., Taira, B.R., Chohan, J., Cardoz, H., Chisena, E., \& Thode, H.C. (2009). Validation of the Wong-Baker FACES pain rating scale in pediatric emergency department patients. Academic Emergency Medicine, 17(1), 50-54.

Gausche-Hill, M., Schmitz, C., \& Lewis, R.J. (2007). Pediatric preparedness of US emergency departments: A 2003 survey. Pediatrics, 120(6), 1229-1237. 
Gelfand, K.M., \& Dahlquist, L.M. (2003). An examination of the relation between child distress and mother and nurse verbal responses during pediatric oncology procedures. Children's Health Care, 32(4), 257-272.

Gordon, D.B., Pellino, T.A., Miaskowski, C., McNeill, J.A., Paice, J.A., Laferriere, D., \& Bookbinder, M. (2002). A 10-year review of quality improvement monitoring in pain management: Recommendations for standardized outcome measures. Pain Management Nursing, 3(4), 116-130.

Gourde, J., \& Damian, F.J. (2012). ED fracture pain management in children. Journal of Emergency Nursing, 38(1), 91-97.

Graham, P., \& Hardy, M. (2004). The immobilization and restraint of paediatric patients during plain film radiographic examinations. Radiography, 10, 23-31.

Grant, K., \& Crouch, R. (2011). Who should nurse children requiring emergency care? International Emergency Nursing, 19, 209-211.

Greipp, M.E. (1992). Undermedication for pain: An ethical model. Advances in Nursing Science, 15(1), 44-53.

Guba, E., \& Lincoln, Y. (1994). Competing paradigms in qualitative research. In N.Denzin and Y. Lincoln (Eds.), Handbook of qualitative research (pp. 105-117). Thousand Oaks, CA: Sage Publications.

Guru, V., \& Dubinsky, I. (2000). The patient vs. caregiver perception of acute pain in the emergency department. The Journal of Emergency Medicine, 18(1), 7-12.

Hamdy, R.C. (2001). The decade of pain control and research. Southern Medical Journal, 94(8), 753-754.

Hamers, J.P.H., Abu-Saad, H.H., Halfens, R.J.G., \& Schumacher, J.N.M. (1994). Factors 
influencing nurses' pain assessment and interventions in children. Journal of Advanced Nursing, 20, 853-860.

Hammersley, M., \& Atkinson, P. (1983). Ethnography principles in practice. New York: Tavistock Publications.

Hemmelgarn, A.L., Glisson, C., \& Dukes, D. (2001). Emergency room culture and the emotional support component of family-centered care. Children's Health Care, 30(2), 93-110.

Hennes, H., Kim, M.K., \& Pirrallo, R.G. (2005). Prehospital pain management: A comparison of providers' perceptions and practices. Prehospital Emergency Care, $9(1), 32-39$.

Hermann, C., Hohmeister, J., Demirakca, S., Zohsel, K., \& Flor, H. (2006). Long-term alteration of pain sensitivity in school-aged children with early pain experiences. Pain, 125(3), 278-285.

Herr, K., Coyne, P.J., Key, T., Manworren, R., McCaffery, M., Merkel, S., Pelosi-Kelly, J., \& Wild, L. (2006). Pain assessment in the nonverbal patient: Position statement with clinical practice recommendations. Pain Management Nursing, 7(2), 44-52.

Herr, J., Coyne, P.J., McCaffery, M., Manworren, R., \& Merkel, S. (2011). Pain assessment in the patient unable to self-report: Position statement with clinical practice recommendations. Pain Management Nursing, 12(4), 230- 250.

Hester, N.O. (1995). Integrating pain assessment and management into the care of children with cancer. In D.B. McGuire, C.H. Yarbo, \& B.R. Ferrell, Cancer Pain Management (2 ${ }^{\text {nd }}$ Ed., pp. 231-271). Boston: Jones and Bartlett Publishers.

Hine, K. (2007). The use of physical restraint in critical care. Nursing in Critical Care, 
12(1), 6-11.

Howell, D., Butler, L., Vincent, L., Watt-Watson, J., \& Stearns, N. (2000). Influencing nurses' knowledge, attitudes, and practice in cancer pain management. Cancer Nursing an International Journal for Cancer Care, 23(1), 55-63.

Hunt, A. (2006). Pain: Assessment. In A. Goldman, R. Hain, and S. Liben (Eds.), Oxford textbook of palliative care for children (pp.281-303). Oxford, England: Oxford University Press.

Hull, K., \& Clarke, D. (2010). Restraining children for clinical procedures: A review of the issues. British Journal of Nursing, 19(6), 346-350.

Huth, M.M. \& Moore, S.M. (1998). Prescriptive theory of acute pain management in infants and children. JSPN, 3(1), 23-32.

Hwang, U., Richardson, L.D., Sonuyi, T.O., \& Morrison, R.S. (2006). The effect of emergency department crowding on the management in older adults with hip fracture. JAGS, 54, 270-275.

Hwang, U., Richardson, L., Livote, E., Harris, B., Spencer, N., \& Morrison, R.S. (2008). Emergency department crowding and decreased quality of pain care. Academic Emergency Medicine, 15(12), 1248-1255.

IBM Software Package for Social Sciences (SPSS) (Version 20.0) [Computer software]. Idell, C.S., Grant, M., \& Kirk, C. (2007). Alignment of pain reassessment practices and national comprehensive cancer network guidelines. Oncology Nursing Forum, 34(3), $661-671$.

International Association for the Study of Pain. (1979). Pain terms: A list with definitions andnotes on usage: Recommended by the International Association for 
the Study of Pain Subcommittee on Taxonomy. Pain, 6, 249.

Jacob, E., \& Puntillo, K.A. (1999). Pain in hospitalized children: Pediatric nurses' beliefs and practices. Journal of Pediatric Nursing, 14(6), 379-391.

Jacox, A., Ferrell, B., Heidrich, G., Hester, N., \& Miaskowski, C. (1992). A guide for the nation: Managing acute pain. American Journal of Nursing, 92(5), 49-55.

Jadav, M.A.R., Lloyd, G., McLauchlan, C., \& Hayes, C. (2009). Routine pain scoring does not improve analgesia provision for children in the emergency department. Emergency Medicine Journal, 26, 695-697.

Jay, S.M. (1988). Invasive medical procedures: Psychological intervention and assessment. In D.K. Routh (Ed.), Handbook of Pediatric Psychology (pp. 401-425). New York, N.Y.: The Guilford Press.

Jeffery, K. (2002). Therapeutic restraint of children: It must always be justified. Paediatric Nursing, 14(9), 20-22.

Jeffery, K. (2010). Supportive holding or restraint: Terminology and practice. Paediatric Nursing, 22(6), 24-28.

Johnson, S.H. (2005). The social, professional, and legal framework for the problem of pain management in emergency medicine. The Journal of Law, Medicine, \& Ethics, 33(4), 741-760.

Johnston, C.C., Gagnon, A.J., Fullerton, L., Common, C., Ladores, M., \& Forlini, S. (1998). One-week survey of pain intensity on admission and discharge from the emergency department: A pilot study. The Journal of Emergency Medicine, 16(3), $377-382$.

Johnston, C.C., Bournaki, M.C., Gagnon, A.J., Pepler, C.J., \& Bourgault, P. (2005). Self- 
reported pain intensity and associated distress in children aged 4-18 years on admission, discharge, and one-week follow up to emergency department. Pediatric Emergency Care, 21(5), 342-346.

Joint Commission on Accreditation of Healthcare Organizations (JCAHO). (1999). Standards for pain assessment and treatment. Comprehensive accreditation manual for ambulatory care, behavioral healthcare, health care networks, home care, hospitals, and long term care. Oakbrook, IL: JCAHO.

Joint Commission on Accreditation of Health Care Organizations. (2000).

Comprehensive accreditation manual for hospitals. Oakbrook, IL: Joint Commission Resources; 2000. TX: 7.5.3

Jones, G.E., \& Machen, I. (2003). Pre-hospital pain management: The paramedics’ perspective. Accident and Emergency Nursing, 11, 166-172.

Juhl, G.A., and Conners, G.P. (2005). Emergency physicians' practices and attitudes regarding procedural anaesthesia for nasogastric tube insertion. Emergency Medicine Journal, 22, 243-245.

Kaasalainen, S., Coker, E., Dolovich, L., Papaioannou, A., Hadjistavropoulos, R., Emili, A., \& Ploeg, J. (2007). Pain management decision making among long term care physicians and nurses. Western Journal of Nursing Research, 29(5), 561-580.

Kalisch, B.J. (2006). Missed nursing care: A qualitative study. Journal of Nursing Care Quality, 21(4), 306-313.

Kaplan, C.P., Sison, C., \& Platt, S.L. (2008). Does a pain scale improve pain assessment in the pediatric emergency department? Pediatric Emergency Care, 24(9), 605- 608. Kelly, A.M., Powell, C.V., \& Williams, A. (2002). Parent visual analogue scale ratings of 
children's pain do not reliably reflect pain reported by child. Pediatric Emergency Care, 18 (3), 159-162.

Kennedy, R.M., \& Luhmann, J.D. (1999). The “ouchless emergency department” getting closer: Advances in decreasing distress during painful procedures in the emergency department. Pediatric Clinics of North America, 46(6), 1215-1247.

Kennedy, S., \& Mohr, W. (2001). A prolegomenon on restraint of children: Implicating constitutional rights. American Journal of Orthopsychiatry, 71, 26-37.

Kim, M.K., Galustyan, S., Sato, T.T., Bergholte, J., \& Hennes, H.M. (2003). Analgesia for children with acute abdominal pain: A survey of pediatric emergency physicians and pediatric surgeons. Pediatrics, 112(5), 1122-1126.

Kissoon, N., McGrath, P.A., \& Glebe, D. (1990). Children's responses to painful procedures in the emergency department (ED). Pain, 41 (S1), PS7.

Kleiber, C., Jennissen, C., McCarthy, A.M., \& Ansley, T. (2011). Evidence-based pediatric pain management in emergency departments of a rural state. The Journal of Pain, 12(8), 900-910.

Kluckhohn, F.R., \& Strodbeck, F.L. (1973). Variations in value orientations. Westport, Connecticut: Greenwood Press.

Kneafsey, R. (2000). The effect of occupational socialization on nurses' patient handing practices. Journal of Clinical Nursing, 9, 585-593.

Koch, T. (2006). Establishing rigour in qualitative research: The decision trail. Journal of Advanced Nursing, 53(1), 91-103.

Koch, T., \& Harrington, A. (1998). Reconceptualizing rigour: The case for reflexivity. Journal of Advanced Nursing, 28(4), 882-890. 
Kolcaba, K. (2003). Comfort theory and practice: A vision for holistic health care and research. New York, NY: Springer Publishing Company, Inc

Kontio, R., Valimakki, M., Putkonen., H., Kuosmanen, L. Scott, A., \& Joffe, G. (2010). Patient restrictions: Are there ethical alternatives to seclusion and restraint? Nursing Ethics, 17(1), 65-76.

Kortesluoma, R.L., \& Nikkonen, M. (2004). 'I had this horrible pain': The sources and causes of pain experiences in 4 to 11 - year-old hospitalized children. Journal of Child Health Care, 8(3), 210-231.

Kotzer, A.M. (2000). Factors predicting postoperative pain in children and adolescents following spine fusion. Issues in Comprehensive Pediatric Nursing, 23, 83-102.

Krauss, B. (2001). Management of acute pain and anxiety in children undergoing procedures in the emergency department. Pediatric Emergency Care, 17(2), 115-122.

Krebel, M.S., Clayton, C., \& Graham, C. (1996). Child life programs in the pediatric emergency department. Pediatric Emergency Care, 12(1), 13-15.

Kumar, N. (2007). WHO normative guidelines on pain management report of a delphi study to determine the need for guidelines and to identify the number and topics of guidelines that should be developed by WHO. Retrived March 12, 2010 from http://www.who.int/medicines/areas/quality_safety/delphi_study_pain_guidelines.pdf

Lambrenos, K., \& McArthur, E. (2003). Introducing a clinical holding policy. Paediatric Nursing, 15(4), 30-33.

Lassetter, J.H. (2006). The effectiveness of complementary therapies on the pain experience of hospitalized children. Journal of Holistic Nursing, 24(3), 196-208.

Leininger, M.M. (1970). Nursing and anthropology: Two worlds to blend. New York: 
John Wiley \& Sons.

Leininger, M. (1995). Transcultural nursing concepts, theories, research and practices ( $2^{\text {nd }}$ ed.). New York: McGraw-Hill.

Leininger, M.M., \& McFarland, M.R. (2006). Culture care diversity and universality a worldwide nursing theory ( $2^{\text {nd }}$ ed.). Boston: Jones and Bartlett Publishers, Inc.

Lewis, M., Taylor, K., \& Parks, J. (2009). Crisis prevention management: A program to reduce the use of seclusion and restraint in inpatient mental health setting. Issues in Mental Health Nursing, 30(3), 159-164.

Lincoln, Y., \& Guba, E. (1985). Naturalistic inquiry. Newbury Park, CA: Sage Publications.

Liossi, C. (2002). Procedure-related cancer pain in children. United Kingdom: Radcliffe Medical Press.

Lipson, J.G. (1991). The use of self in ethnographic research. In J.M. Morse (Ed.), Qualitative nursing research: A contemporary dialogue (Rev. ed.). Newbury Park: Sage Publications.

Locke, R., Stefano, M., Koster, A., Taylor, B., \& Greenspan, J. (2011). Optimizing patient/caregiver satisfaction through quality of communication in the pediatric emergency department. Pediatric Emergency Care, 27(11), 1016-1021.

Lofland, J., Snow, D., Anderson, L., \& Lofland, L.H. (2006). Analyzing social settings: A guide to qualitative observation and analysis ( $4^{\text {th }}$ ed.). Belmont, CA: Wadsworth/ Thomson Learning.

Lowenthal, B. (1978). Piaget's preoperational stage of development and applications for special preschoolers. In R. Weizmann, R. Brown, P.J. Levinson, and P.A. Taylor 
(Eds.), Piagetian Theory and its Implications for the Helping Professions Volume 1 (pp. 308-314). Los Angeles, California: University of Southern California. Ludwig-Beymer, P. (2008). Transcultural aspects of pain. In M.M. Andrews and J.S. Boyle, Trancultural Concepts in Nursing Care (5 ${ }^{\text {th }}$ ed., pp.329-354). Philadelphia: Wolters Kluwer Health Lippincott Williams and Wilkins.

Maciocia, P.M., Strachan, E.M., Akram, A.R., Hendrie, R.E., Kelly, D.N., Kemp, A., McLukie, A.M., Smith, L.M., \& Beattie, T.F. (2003). Pain assessment in the paediatric emergency department: Whose view counts? European Journal of Emergency Medicine, 10(4), 264-267.

Mackenzie, A.E. (1994). Evaluating ethnography: Considerations for analysis. Journal of Advanced Nursing, 19(4), 774-781.

Mackintosh, C. (2006). Caring: The socialization of pre-registration student nurses: A longitudinal qualitative descriptive study. International Journal of Nursing Studies, $43,953-962$.

MacLean, S., Obispo, J., \& Young, K.D. (2007). The gap between pediatric emergency department procedural pain management treatments available and actual practice. Pediatric Emergency Care, 23(2), 87-93.

Magaret, N.D., Clark, T.A., Warden, C.R., Magnusson, A.R., \& Hedges, J.R. (2002).

Patient satisfaction in the emergency department- A survey of pediatric patients and their parents. Academic Emergency Medicine, 9(12), 1379-1387.

Manimala, M.R., Blount, R.L., \& Cohen, L.L. (2000). The effects of parental reassurance versus distraction on child distress and coping during immunizations. Children's Health Care, 29(3), 161-177. 
Manne, S.L., Bakeman, R., Jacobsen, P.B., Gorfinkle, K., Bernstein, D., \& Redd, W.H. (1992). Adult-child interaction during invasive medical procedures. Health Psychology, 11(4), 241-249.

Manworren, R.C.B. (2000). Pediatric nurses' knowledge and attitudes survey regarding pain. Pediatric Nursing, 26(6), 610-614.

Manworren, R.C.B. (2001). Development and testing of the Pediatric Nurses’ Knowledge and Attitudes Survey Regarding pain. Pediatric Nursing, 27(2), 151-158.

Manworren, R.C.B., \& Hynan, L.S. (2003). Clinical validation of FLACC: Preverbal patient pain scale. Pediatric Nursing, 29(2), 140-146.

Margolius, F.R., Hudson, K.A., \& Michel, Y. (1995). Beliefs and perceptions about children in pain: A survey. Pediatric Nursing, 21(2), 111-115.

Matthews, E., \& Malcolm, C. (2007). Nurses' knowledge and attitudes in pain management practices. British Journal of Nursing, 16(3), 174-179.

Matza, D. (1969). Becoming deviant. Englewood, NJ: Prentice Hall.

May, S.L., Johnston, C.C., Choinière, M., Fortin, C., Kudirka, D., Murray, L., \& Chalut, D. (2009). Pain management practices in a pediatric emergency room (PAMPER) study. Pediatric Emergency Care, 25(8), 498-503.

McCaffery, M., \& Ferrell, B.R. (1997). Nurses’ knowledge of pain assessment and management: How much progress have we made? Journal of Pain and Symptom Management, 14(3), 175-188.

McCaffery, M., Ferrell, B.R., \& Pasero, C. (2000). Nurses’ personal opinions about patients' pain and their effect on recorded assessments and titration of opioid doses. Pain Management Nursing, 1(3), 79-87. 
McCaffery, M. (2002). What is the role of nondrug methods in the nursing care of patients with acute pain? Pain Management Nursing, 3(3), 77-80.

McCarthy, A.M., Kleiber, C., Hanrahan, K., Zimmerman, M.B., Westhus, N., \& Allen, S. (2010). Impact of parent-provided distraction on child responses to an IV insertion. Children's Health Care, 39, 125-141.

McCloskey, J.C., \& Bulechek, G.M. (Eds.). (1996). Iowa intervention project: Nursing intervention classification (NIC), (2 ${ }^{\text {nd }}$ ed.). St Louis: Mosby Year Book.

McConahay, T., Bryson, M., \& Bulloch, B. (2006). Defining mild, moderate, and severe pain by using the color analogue scale with children presenting to a pediatric emergency department. Academic Emergency Medicine, 13, 341-344.

McErlean, M., Bartfield, J.M., Karunakar, T.A., Whitman, M.C.W., \& Turley, D.M. (2003). Midazolam syrup as a premedication to reduce the discomfort associated with pediatric intravenous catheter insertion. Journal of Pediatrics, 142, 429-430.

McGlone, R.G., Ranasinghe, S., \& Durham, S. (1998). An alternative to "brutacaine": A comparison of low dose intramuscular ketamine with intranasal midazolam in children before suturing. Journal of Accident Emergency Medicine, 15, 231-236.

Meadows, K. (2003). So you want to do research 1: An overview of the research process. British Journal of Community Nursing, 8(8), 369-375.

Meehan, D.A., McRae, M.E., Rourke, D.A., Eisenburg, C., \& Imperial, F.A. (1995). Analgesic administration, pain intensity, and patient satisfaction in cardiac surgical patients. American Journal of Critical Care, 4(6), 435-442.

Melhuish, S. \& Payne, H. (2006). Nurses' attitudes to pain management during routine venepuncture in young children. Paediatric Nursing, 18(2), 20-23. 
Melzack, R. (2001). Pain and the neuromatrix in the brain. Journal of Dental Education, 65(12), 1378-1382.

Meunier-Sham, J., \& Ryan, K. (2003). Reduing pediatric pain during ED procedures with a nurse-driven protocol: An urban pediatric emergency department's experience. Journal of Emergency Nursing, 29(2), 127-132.

Miner, J., Biros, M.H., Trainor, A., Hubbard, D., \& Beltram, M. (2006). Patient and physician perceptions as risk factors for oligoanalgesia: A prospective observational study of the relief of pain in the emergency department. Academic Emergency Medicine, 13(2), 140-146.

Mohr, W.K., Petti, T.A., \& Mohr, B.D. (2003). Adverse effects associated with physical restraint. Canadian Journal of Psychiatry, 48(5), 330-337.

Mooney, M. (2007). Professional socialization: The key to survival as a newly qualified nurse. International Journal of Nursing Practice, 13, 75-80.

Morse, J.M. (Ed.). (1991). Qualitative nursing research: A contemporary dialogue (Rev. ed.). Newbury Park, CA: Sage Publications.

Munhall, P.L. (2007). Nursing research: A qualitative perspective $\left(4^{\text {th }}\right.$ ed.). Sudbury, MA: Jones and Bartlett Publishers.

Naber, S.J., Halstead, L.K., Broome, M.E., \& Rehwaldt, M. (1995). Communication and control: Parent, child, and health care professional interactions during painful procedures. Issues in Comprehensive Pediatric Nursing, 18, 79-90.

Nawar, E.W., Niska, R.W., \& Xu, J. (2007, June). National hospital ambulatory medical care survey: 2005 emergency department summary. Advance Data from Vital \& Health Statistics Centers for Disease Control, 386, 1-32. 
Nelson, D.S., Hoagland, J.R., Kunkel, N.C. (2000). Costs of sedation using oral midazolam: Money, time, and parental attitudes. Pediatric Emergency Care, 16(2), 80-84.

New Jersey Hospital Association. (2010). 'What will happen to my hospital?' It's a critical time for hospitals and their patients. Retrived from http://www.njha.com/advocacy/pdf/hospital_closures_next.pdf

Nyström, M., Dahlberg, K., \& Carlsson, G. (2003). Non-caring encounters at a n emergency care unit- a life-world hermeneutic analysis of an efficiency-driven organization. International Journal of Nursing Studies, 40, 761-769.

Nyström, M., Nyden, K., \& Petersson, M. (2003). Being a non-urgent patient in an emergency care unit-a strive to maintain personal integrity. Accident and Emergency Nursing, 11, 22-26.

O’Donnell, J., Ferguson, L.P., \& Beattie, T.F. (2002). Use of analgesia in a paediatric accident and emergency department following limb trauma. European Journal of Emergency Medicine, 9(1), 5-8.

Olive, P. (2003). The holistic nursing care of patients with minor injuries attending the A\&E department. Accident and Emergency Nursing, 11, 27-32.

Pack-Mabien, A., Labbe, E., Herbert, D., \& Haynes Jr. J. (2001). Nurses' attitudes and practices in sickle cell pain management. Applied Nursing Research, 14(4), 187-192.

Pagnamenta, R., \& Benger, J.R. (2008). Factors influencing parent satisfaction in a children's emergency department: Prospective questionnaire- based study. Emergency Medicine Journal, 25, 417-419.

Pasek, T.A., Thomas, D., Khimji, I., Schmitt, C., Spence, A., \& Hanni, R. (2007). 
Implementation of a nurse-driven topical analgesic protocol: Two steps forward, one step back. Pediatric Pain Letter, 9(3). Retrived from http://childpain.org/ppl/issues/V9n3_2007/v9n3_pasek.shtml

Pate, J.T., Blount, R.L., Cohen, L.L., \& Smith, A.J. (1996). Childhood medical experience and temperament as predictors of adult functioning in medical situations. Children's Health Care, 25, 281-296.

Pearch, J. (2005). Restraining children for clinical procedures. Paediatric Nursing, 17(9), 36-38.

Phillips, D.M., \& Joint Commission on Accreditation of Healthcare Organizations. (2000). JCAHO pain management standards unveiled. Journal of the American Medical Association (JAMA), 284(4), 428-429.

Philpin, S.M. (1999). The impact of 'Project 2000' educational reforms on the occupational socialization of nurses: An exploratory study. Journal of Advanced Nursing, 29(6), 1326-1331.

Pines, J.M., \& Hollander, J.E. (2008). Emergency department crowding is associated with poor care for patients with severe pain. Annals of Emergency Medicine, 51(1), $1-5$.

Pines, J.M., Shofer, F.S., Isserman, J.A., Abbuhl, S.B., \& Mills, A.M. (2010). The effect of emergency department crowding on analgesia in patients with back pain in two hospitals. Academic Emergency Medicine, 17(3), 276-283.

Pokela, M.L. (1994). Pain relief can reduce hypoxemia in distressed neonates during routine treatment procedures. Pediatrics, 93(3), 379-383.

Pölkki, T., Laukkala, H., Vehviläinen-Julkunen, K., \& Pietilä, A.M. (2003). Factors 
influencing nurses' use of nonpharmacological pain alleviation methods in paediatric patients. Scandinavian Journal of Caring Science, 17, 373-383.

Pole, C., \& Morrison, M. (2003). Ethnography for education. England: Open University Press.

Polit, D.F., \& Beck, C.T. (2008). Nursing research: Generating and assessing evidence for nursing practice $\left(8^{\text {th }}\right.$ ed.). Philadelphia: Wolter Kluwer/ Lippincott Williams and Wilkins.

Polomano, R.C., Dunwoody, C.J., Krenzischek, D.A., \& Rathmell, J.P. (2008).

Perspective on pain management in the $21^{\text {st }}$ century. Pain Management Nursing, 9(1), S3-S10.

Porter, G., \& Chapman, R. (2004, September). Assessment and management of pain in children in the emergency department. Abstracts $1^{\text {st }}$ National Emergency Nursing Conference, Adelaide.

Potter, P., Deshields, T., Kuhrik, M. (2010). Delegation practices between registered nurses and nursing assistive personnel. Journal of Nursing Management, 18, 157165.

Probst, B.D., Lyons, E., Leonard, D., \& Esposito, T.J. (2005). Factors affecting emergency department assessment and management of pain in children. Pediatric Emergency Care, 21(5), 298-305.

Puls-McColl, P.J., Holden, J.E., \& Buschmann, M.T. (2001). Pain management: An assessment of surgical nurses' knowledge. MedSurg Nursing, 10(4), 185-191.

Puntillo, K., Neighbor, M., O’Neil, N., \& Nixon, R. (2003). Accuracy of emergency nurses in assessment of patients' pain. Pain Management Nursing, 4(4), 171-175. 
Rajasagaram, U., Taylor, D.M., Braitberg, G., Pearsell, J.P., \& Capp, B.A. (2009).

Paediatric pain assessment: Differences between triage nurse, child, and parent. Journal of Paediatrics and Child Health, 45, 199-203.

Ramponi, D. (2009). Reducing pain in pediatric procedures in the emergency department. Journal of Emergency Nursing, 35(4), 379-382.

Rasmussen, J.K., Frederiksen, J.A., Hallonsten, A., \& Poulsen, S. (2005). Danish dentists' knowledge, attitudes, and management of procedural dental pain in children: Association with demographic characteristics, structural factors, perceived stress during the administration of local analgesia and their tolerance towards pain. International Journal of Paediatric Dentistry, 15, 159-168.

Read, J.V. (1994). Perceptions of nurses and physicians regarding pain management of pediatric emergency room patients. Pediatric Nursing, 20(3), 314-318.

Reynolds, D. (2001). Parents sue facility and staff for boy's restraint death. Inclusion Daily Express. Retrived from http://www.inclusiondaily.com/news/institutions/ia/iowa.htm

Riva, P., Rusconi, P., Montali, L., \& Cherubini, P. (2011). The influence of anchoring on pain judgement. Journal of Pain and Symptom Management, 42(2), 265-275.

Robertson, M.H.B., \& Boyle, J.S. (1984). Ethnography: Contributions to nursing research. Journal of Advanced Nursing, 9, 43-49.

Robinson, S., \& Collier, J. (1997). Holding children still for procedures. Paediatric Nursing, 9(4), 12-14.

Rogers, T.L., \& Ostrow, C.L. (2004). The use of EMLA cream to decrease venipuncture pain in children. Journal of Pediatric Nursing: Nursing Care of Children and 
Families, 19(1), 33-39.

Rømsing, J., Møller-Sonnergaard, J., Hertel, S., \& Rasmussen, M. (1996). Postoperative pain in children: Comparison between ratings of children and nurses. Journal of Pain and Symptom Management, 11(1), 42-46.

Roper, J.M., \& Shapira, J. (2000).Ethnography in nursing research. Thousand Oaks, CA: Sage Publications.

Royal Australasian College of Physicians. (2006). Management of procedure-related pain in children and adolescents. Journal of Paediatrics and Child Health, 42, S1-S29.

Rupp, T., \& Delaney, K.A. (2004). Inadequate analgesia in emergency medicine. Annals of Emergency Medicine, 43, 494-503.

Russo, T.A. (2010). Factors affecting the process of clinical decision-making in pediatric pain management by emergency department nurses (Doctoral dissertation). Available from ProQuest Dissertations and Theses database. (UMI No. 3424489)

Ryan, F., Coughlan, M., \& Cronin, P. (2007). Step-by-step guide to critiquing research. Part 2: Qualitative research. British Journal of Nursing, 16(12), 738-744.

Saarnio, R., \& Isola, A. (2009). Use of physical restraint in institutional elderly care in Finland: Perspectives of patients and their family members. Research in Gerontological Nursing, 2(4), 276-286.

Salanterä, S. (1999). Finnish nurses' attitudes to pain in children. Journal of Advanced Nursing, 29(3), 727-736.

Saldaña, J. (2009). The coding manual for qualitative researchers. Thousand Oaks, CA: Sage Publications.

Sandelowski, M., \& Barroso, J. (2002). Reading qualitative studies. International Journal 
of Qualitative Methods, 1(1), Retrived from http://www.ualberta.ca/ ijqm

Santervas, Y.F., Cotanda, C.P., Carretero, L.M., Garcia, V.L., Sainz de la Maza, V.T., \& Cubells, C.L. (2010). Impact of a program to improve pain management in an emergency department. European Journal of Emergency Medicine, 17, 110-112.

Schafheutle, E.I., Cantrill, J.A., \& Noyce, P.R. (2001). Why is pain management suboptimal on surgical wards? Journal of Advanced Nursing, 33(6), 728-737.

Schechter, N.L. (1989). The undertreatment of pain in children: An overview. In N.L. Schechter (Ed.), Acute Pain in Children: The Pediatric Clinics of North America, 36(4), 781-794. Philadelphia: W.B. Saunders Company.

Schechter, N.L., Berde, C.B., \& Yaster, M. (Eds.). (2003). Pain in infants, children, and adolescents ( $2^{\text {nd }}$ ed.). Philadelphia, PA: Lippincott Williams and Wilkins.

Schechter, N.L. (2008). From the ouchless place to comfort central: The evolution of a concept. Pediatrics, 122 (S3), S154-S160.

Schein, E.H. (1992). Organizational culture and leadership ( $2^{\text {nd }}$ ed.). San Francisco: Jossey-Bass Publishers.

Schmidt, K., Holiday, D., Kleiber, C., Peterson, M., \& Phearman, L. (1994). Implementation of the AHCPR pain guidelines for children. Journal of Nursing Care Quarterly, 8(3), 68-74.

Schmidt, I. \& Svarstad, B. (2002). Nurse-physician communication and quality of drug use in Swedish nursing homes. Social Science Medicine, 54, 1767-1777.

Schultz, A.A., Strout, T.D., Jordan, P., \& Worthing, B. (2002). Safety, tolerability, and efficacy of iontophoresis with lidocaine for dermal anesthesia in ED pediatric patients. Journal of Emergency Nursing, 28(4), 289-296. 
Seidel, J.V. (1998). Qualitative data analysis. Retrived from www.qualisresearch.com (originally published as Qualitative data analysis, in The Ethnograph v5.0: A users guide, Appendix E, 1998, Colorado Springs, Colorado: Qualis Research.

Selekman, J. \& Snyder, B. (1996). Uses of and alternatives to restraints in pediatric settings. AACN Clinical Issues, 7(4), 603-610.

Selekman, J., \& Snyder, B. (1997). Institutional policies on the use of physical restraints on children. Pediatric Nursing, 23(5), 531-537.

Shavit, I., Kofman, M., Leder, M., Hod, T., \& Kozer, E. (2009). Observational pain assessment versus self-report in paediatric triage. Emergency Medicine Journal, 25(9), 552-555.

Shimahara, N. (1984). Anthroethnography: A methodological consideration. Journal of Thought, 19(2), 61-74

Siegel, L.J. (1988). Dental treatment. In D.K. Routh (Ed.), Handbook of pediatric psychology (pp. 448-459). New York: Guilford Press.

Simpson, K., Kautzman, L., \& Dodd, S. (2002). The effects of a pain management education program on the knowledge level and attitudes of clinical staff. Pain Management Nursing, 3(3), 87-93.

Singer, A.J., Richman, P.B., Kowalska, A., \& Thode, H.C. (1999). Comparison of patient and practitioner assessments of pain from commonly performed emergency department procedures. Annals of Emergency Medicine, 33(6), 652-658.

Singer, A.J., Gulla, J., \& Thode, H.C. (2002). Parents and practitioners are poor judges of young children's pain severity. Academic Emergency Medicine, 9(6), 609-612. 
Sinha, M., Christopher, N.C., Fenn, R., \& Reeves, L. (2006). Evaluation of nonpharmacological methods of pain and anxiety management for laceration repair in the pediatric emergency department. Pediatrics, 117(4), 1162-1168.

Slifer, K.J., Hankinson, J.C., Zettler, M.A., Frutchey, R.A., Hendricks, M.C., Ward, C.M., \& Reesman, J. (2011). Distraction, exposure therapy, counterconditiong, and topical anesthetic for acute pain management during needle sticks in children with intellectual and developmental disabilities. Clinical Pediatrics, 50 (8), 688- 697.

Smith, M., \& Feied, C. (1999). The emergency department as a complex system. The George Washington University School of Medicine and Health Sciences: Mark Smith \& Craig Feied. Retrived from http://necsi.org/projects/yaneer/emergencydeptcx.pdf

Spradley, J.P. \& McCurdy D.W. (1972). The cultural experience: Ethnography in complex society. Chicago: Science Research Associates, Inc.

Spradley, J.P. (1980). Participant observation. New York: Holt, Rinehart, and Winston. Stevenson, M.D., Bivins, C.M., O’Brien, K, \& Gonzalez del Rey, J.A. (2005). Child life intervention during angiocatheter insertion in the pediatric emergency department. Pediatric Emergency Care, 21(11), 712-718.

Stewart, B., Lancaster, G., Lawson, J., Williams, K., \& Daly, J. (2004). Validation of the Alder Hey triage pain score. Archives of Disease in Childhood, 89, 625-630.

Strout, T.D., \& Baumann, M.R. (2011). Reliability and validity of the modified preverbal, early verbal pediatric pain scale in emergency department pediatric patients. International Emergency Nursing, 19, 178- 185.

Stumbo, N.J. (2002). Implication of pain management for therapeutic recreation services. 
Annual in Therapeutic Recreation, 11(31), 106-107, 110.

Sweet, S.D., \& McGrath, P.J. (1998). Relative importance of mothers' versus medical staffs' behavior in the prediction of infant immunization pain behavior. Journal of Pediatric Psychology, 23(4), 249-256.

Tanabe, P. (1996). Pain management practices in the emergency department (Doctoral dissertation) Available from ProQuest Dissertations and Theses database. (UMI No. 9713341)

Tanabe, P., \& Buschmann, M. (1999). A prospective study of ED pain management practices and the patient's perspective. Journal of Emergency Nursing, 25(3), 171177.

Tanabe, P., \& Buschmann, M. (2000). Emergency nurses' knowledge of pain management principles. Journal of Emergency Nursing, 26(4), 299-305.

Taylor, C. (1997). Problem solving in clinical nursing practice. Journal of Advanced Nursing, 26, 329-336.

Terndrup, T.E. (1996). Pediatric pain control. Annals of Emergency Medicine, 27(4), 466470.

Testad, I., Aasland, A.M., \& Aarsland, D. (2005). The effect of staff training on the use of restraint in dementia: A single-blind randomized controlled trial. International Journal of Geriatric Psychiatry, 20(6), 587-590.

Tobin, G.A., \& Begley, C.M. (2004). Methodological rigour within a qualitative framework. Journal of Advanced Nursing, 48(4), 388-396.

Todd, K.H., Ducharme, J., Choiniere, M., Crandall, C.S., Fosnocht, D.E., Homel, P., \& Tanabe, P. (2007). Pain in the emergency department: Results of the Pain and 
Emergency Medicine Initiative (PEMI) multicenter study. The Journal of Pain, 8(6), 460-466.

Tomlinson, D. (2004). Physical restraint during procedures: Issues and implications for practice. Journal of Pediatric Oncology Nursing, 21(5), 258-263.

Tse, M.M., \& Chan, B.S.H. (2004). Knowledge and attitudes in pain management: Hong Kong Nurses' perspective. Journal of Pain and Palliative Care Pharmacotherapy, $18(1), 47-58$.

Twycross, A. (1999). Pain management: A nursing priority. Journal of Child Health Care, 3(3), 19-25.

Twycross, A. (2002). Managing pain in children: An observational study. Journal of Research in Nursing, 7(3), 164-178.

Twycross, A. (2007a). Children's nurses' post-operative pain management practices: An observational study. International Journal of Nursing Studies, 44, 869-881.

Twycross, A. (2007b). What is the impact of theoretical knowledge on children's nurses' post-operative pain management practices? An exploratory study. Nurse Education Today, 27, 697-707.

Twycross, A. (2008). Does the perceived importance of a pain management task affect the quality of children's nurses' post-operative pain management practices? Journal of Clinical Nursing, 17, 3205-3216.

Uman, L.S., Chamber, C.T., McGrath, P.J., \& Kisely, S.R. (2006). Psychological interventions for needle-related pain and distress in children and adolescents. Cochrance Database of Systematic Reviews Online, 18(4): CD005179, 1-94. Valler-Jones, T., \& Shinnick, A. (2005). Holding children for invasive procedures: 
Preparing student nurses. Paediatric Nursing, 17(5), 20-22.

Van Niekerk, L.M., \& Martin, F. (2003). The impact of nurse-physician relationship on barriers encountered by nurses during pain management. Pain Management Nursing, 4(1), 3-10.

Vessey, J.A., Carlson, K.L., \& McGill, J. (1994). Use of distraction with children during acute pain experience. Nursing Research, 43(6), 369-372.

Villarruel, A. (1995). Cultural perspective of pain. In M. Leininger, Transcultural Nursing: Concepts, Theories, Research \& Practices (pp. 263-277). New York: McGraw-Hill, Inc.

Vincent, C.V.H. (2005). Nurses' knowledge, attitudes, and practices regarding children's pain. $M C N, 30(3), 177-183$.

Visina, C.E., Chen, J., Gerthoffer, T.D., Biggs, R., \& Ting, D. (2003). Community hospital physician and nurses attitudes about pain management. Journal of Pain and Palliative Care Pharmacotherapy, 17(2), 51-62.

Walker, A.C., Tan, L., \& George, S. (1995). Impact of culture on pain management: An Australian nursing perspective. Holistic Nursing Practice, 9(2), 48-57.

Walsh, M., \& Dolan, B. (1999). Emergency nurses and their perceptions of caring. Emergency Nurse, 7(4), 24-31.

Walsh, B.M., \& Bartfield, J.M. (2006). Survey of parental willingness to pay and willingness to stay for painless intravenous catheter placement. Pediatric Emergency Care, 22(11), 699-703.

Walters, L., Prideaux, D., Worley, P., Greenhill, J., \& Rolfe, H. (2009). What do general practitioners do differently when consulting with a medical student? Medical 
Education, 43, 268-273.

Wilson, V.J., McCormack, B.G., \& Ives, G. (2005). Understanding the workplace culture of a special care nursery. Journal of Advanced Nursing, 50(1), 27-38.

Winskill, R., \& Andrews, D. (2008). Minimizing the ouch- A strategy to minimize pain, fear and anxiety in children presenting to the emergency department. Australasian Emergency Nursing Journal, 11, 184-188.

Wolcott, H.F. (2008). Ethnography a way of seeing. Lanham, MD: AltaMira Press.

Wolf, J., Evans, B., Hewitson, H., \& Perez, P. (2003). An ethnography of the ER: A closer look at information flows, organizational methodologies, work culture, and the role of the unit secretary. Retrived from http://brynevans.com/papers/AnEthnography-of-the-ER.pdf

Wong, E.M.L., Chan, H.M.S., Rainer, T.H., \& Ying, C.S. (2007). The effect of a triage pain management protocol for minor musculoskeletal injury patients in a Hong Kong emergency department. Australasian Emergency Nursing Journal, 10, 64-72.

Woodgate, R., \& Kristjanson, L.J. (1996a). A young child's pain: How parents and nurses 'take care'. International Journal of Nursing Studies, 33(3), 271-284.

Woodgate, R., \& Kristjanson, L.J. (1996b). “Getting better from my hurts”: Toward a model of the young child's pain experience. Journal of Pediatric Nursing, 11(4), $233-242$.

Young, K.D. (2005). Pediatric procedural pain. Annals of Emergency Medicine, 45(2), 160-171.

Young, J.L., Horton, F.M., \& Davidhizar, R. (2006). Nursing attitudes and beliefs in pain assessment and management. Journal of Advanced Nursing, 53(4), 412-421. 
Young, T., Griffin, E., Phillips, E., \& Stanley, E. (2010). Music as distraction in a pediatric emergency department. Journal of Emergency Nursing, 36(5), 472-473.

Zanolin, M.E., Visentin, M., Trentin, L., Saiani, L., Brugnolli,A., \& Grassi, M. (2007). A questionnaire to evaluate the knowledge and attitudes of health care providers on pain. Journal of Pain and Symptom Management, 33(6), 727-736.

Zelter, L.K., Jay, S.M., \& Fisher, D.M. (1989). The management of pain associated with pediatric procedures. Pediatric Clinics of North America, 36(4), 941- 964.

Zempsky, W. T., Cravero, J.P., Committee on Pediatric Emergency Medicine, Section on Anesthesiology \& Pain Medicine. (2004). Relief of pain and anxiety in pediatric patients in emergency medical systems. Pediatrics, 114 (5), 1348-1356. 
Appendix A

\section{ROBERT WOOD JOHNSON}

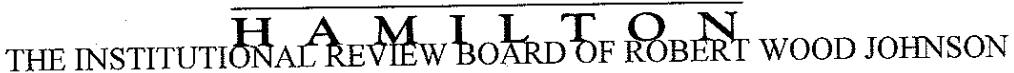 HOSPITAL HAMILTON}

\section{INSTITUTIONAL REVIEW BOARD NOTICE OF APPROVAL}

PRINCIPAL INVESTIGATOR: JoAnn F. Cummings,MSN

TITLE: An Ethnography of the Culture of Pain in a Non-Pediatric Emergency Department SPONSOR/ PROTOCOL NUMBER: College of Nursing Rutgers BIN 326

STUDY STATUS: Open

TYPE OF REVIEW: Full

TYPE OF APPROVAL: New

CONSENT: Modified Version 03/14/2011,Para.5 deleted

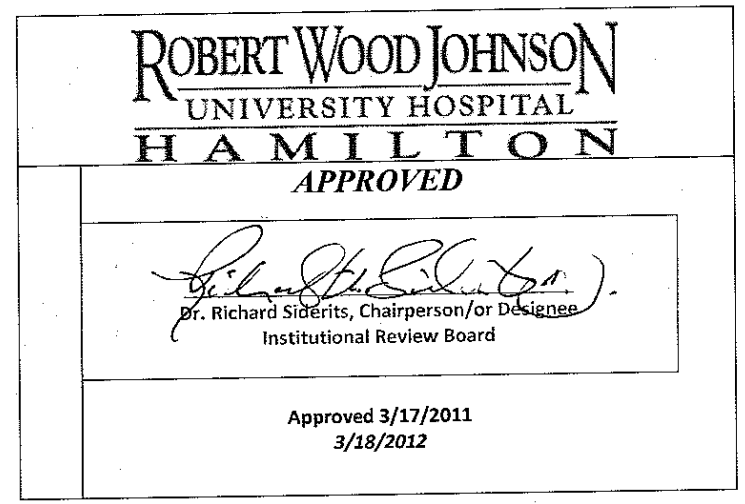

Committee Members

\begin{tabular}{|c|c|c|c|}
\hline Barbara Bromley Wịlliams & Ted Taylor,MDIV,BCC & Monica Olaff & Elizabeth Whetzel, RN, BS,CEN \\
\hline Erondu Ngozii,PharmD & Joyce Schwarz,RN,MPA & Alex Kardos & Lisa Tufkankjian,MD \\
\hline Marc Levine,MD & Ladawna Leeth & Elizabeth Rosvold, MD & \\
\hline Sue Hermida, RN,MSN,NP & Michael Gordon, MD & Jan Stout & \\
\hline
\end{tabular}




\section{Appendix A \\ RUTGERS UNIVERSITY \\ Office of Research and Sponsored Programs \\ ASB III, 3 Rutgers Plaza, Cook Campus \\ New Brunswick, NJ 08901}

March 18, 2011

JoAnn F. Cummings

79 Bates Rd

Jackson NJ 08527

Dear JoAnn Cummings:

(Initial / Amendment / Continuation / Continuation w/ Amendment)
P.I. Name: Cummings

Protocol \#: 11-237M

Protocol Title: "An Ethnography of the Culture of Pain in a Non-Pediatric Emergency Department"

This is to advise you that the above-referenced study has been presented to the Institutional Review Board for the Protection of Human Subjects in Research, and the following action was taken subject to the conditions and explanations provided below:
Approval Date:
$1 / 25 / 2011$
Expiration Date:
$1 / 24 / 2012$
Expedited Category(s):
$5,6,7$
Approved \# of Subject(s):
Open

This approval is based on the assumption that the materials you submitted to the Office of Research and Sponsored Programs (ORSP) contain a complete and accurate description of the ways in which human subjects are involved in your research. The following conditions apply:

- This Approval-The research will be conducted according to the most recent version of the protocol that was submitted. This approval is valid ONLY for the dates listed above;

- Reporting-ORSP must be immediately informed of any injuries to subjects that occur and/or problems that arise, in the course of your research;

- Modifications-Any proposed changes MUST be submitted to the IRB as an amendment for review and approval prior to implementation;

- Consent Form(s)-Each person who signs a consent document will be given a copy of that document, if you are using such documents in your research. The Principal Investigator must retain all signed documents for at least three years after the conclusion of the research;

- Continuing Review-You should receive a courtesy e-mail renewal notice for a Request for Continuing Review before the expiration of this project's approval. However, it is your responsibility to ensure that an application for continuing review has been submitted to the IRB for review and approval prior to the expiration date to extend the approval period;

Additional Notes:

\section{Expedited Approval per 45 CFR 46.110}

\section{Failure to comply with these conditions will result in withdrawal of this approval.}

Please note that the IRB has the authority to observe, or have a third party observe, the consent process or the research itself. The Federal-wide Assurance (FWA) number for the Rutgers University IRB is FWA00003913; this number may be requested on funding applications or by collaborators.

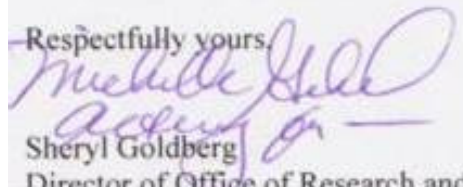

Director of Offide of Research and Sponsored Programs

gibel@grants.rutgers.edu 


\title{
Appendix A
}

\author{
RUTGERS UNIVERSITY \\ Office of Research and Sponsored Programs \\ ASB III, 3 Rutgers Plaza, Cook Campus \\ New Brunswick, NJ 08901
}

March 29, 2011

P.I. Name: Cummings

JoAnn F. Cummings

79 Bates Rd

Jackson NJ 08527

Dear JoAnn Cummings:

Protocol \#: 11-237M

( Initial / Amendment / Continuation / Continuation w/ Amendment )

Protocol Title: "An Ethnography of the Culture of Pain in a Non-Pediatric Emergency Department"

This is to advise you that the above-referenced study has been presented to the Institutional Review Board for the Protection of Human Subjects in Research, and the following action was taken subject to the conditions and explanations provided below:

Amendment to Approval Date: 3/25/2011 Expiration Date: 1/24/2012 Expedited Category(s): 5,6,7

This approval is based on the assumption that the materials you submitted to the Office of Research and Sponsored Programs (ORSP) contain a complete and accurate description of the ways in which human subjects are involved in your research. The following conditions apply:

- This Approval-The research will be conducted according to the most recent version of the protocol that was submitted. This approval is valid ONLY for the dates listed above;

- $\quad$ Reporting-ORSP must be immediately informed of any injuries to subjects that occur and/or problems that arise, in the course of your research;

- Modifications-Any proposed changes MUST be submitted to the IRB as an amendment for review and approval prior to implementation;

- Consent Form(s)-Each person who signs a consent document will be given a copy of that document, if you are using such documents in your research. The Principal Investigator must retain all signed documents for at least three years after the conclusion of the research;

- Continuing Review-You should receive a courtesy e-mail renewal notice for a Request for Continuing Review before the expiration of this project's approval. However, it is your responsibility to ensure that an application for continuing review has been submitted to the IRB for review and approval prior to the expiration date to extend the approval period;

Additional Notes: $\quad$ Administratively Expedited Amendment Approval per 45 CFR 46.110(b)(2) on 3/25/11 for Revised Informed Consent Form to Remove Header Information \& \& Deleted Information

Failure to comply with these conditions will result in withdrawal of this approval.

Please note that the IRB has the authority to observe, or have a third party observe, the consent process or the research itself. The Federal-wide Assurance (FWA) number for the Rutgers University IRB is FWA00003913; this number may be requested on funding applications or by collaborators.

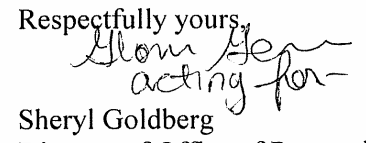

Director of Office of Research and Sponsored Programs

gibel@grants.rutgers.edu

cc: Elise L. Lev 


\author{
Appendix A \\ RUTGERS UNIVERSITY \\ Office of Research and Sponsored Programs \\ ASB III, 3 Rutgers Plaza, Cook Campus \\ New Brunswick, NJ 08901
}

January 24,2012

JoAnn F. Cummings

79 Bates Rd

Jackson NJ 08527

Dear JoAnn Cummings:
P.I. Name: Cummings Protocol \#: 11-237Mx

(Initial / Amendment / Continuation / Continuation w/ Amendment )

Protocol Title: "An Ethnography of the Culture of Pain in a Non-Pediatric Emergency Department"

This is to advise you that the above-referenced study has been presented to the Institutional Review Board for the Protection of Human Subjects in Research, and the following action was taken subject to the conditions and explanations provided below:

$\begin{array}{lllll}\text { Approval Date: } & \text { 11/28/2011 } & \text { Expiration Date: } & 11 / 27 / 2012 & \text { Expedited Category: } 8 \mathrm{c} \\ \text { Approved \# of Subject(s): } & \text { Open } & \text { Currently Enrolled: } & 84 & \end{array}$

This approval is based on the assumption that the materials you submitted to the Office of Research and Sponsored Programs (ORSP) contain a complete and accurate description of the ways in which human subjects are involved in your research. The following conditions apply:

- This Approval-The research will be conducted according to the most recent version of the protocol that was submitted. This approval is valid ONLY for the dates listed above;

- Reporting-ORSP must be immediately informed of any injuries to subjects that occur and/or problems that arise, in the course of your research;

- Modifications-Any proposed changes MUST be submitted to the IRB as an amendment for review and approval prior to implementation;

- Consent Form(s)-Each person who signs a consent document will be given a copy of that document, if you are using such documents in your research. The Principal Investigator must retain all signed documents for at least three years after the conclusion of the research;

- Continuing Review-You should receive a courtesy e-mail renewal notice for a Request for Continuing Review before the expiration of this project's approval. However, it is your responsibility to ensure that an application for continuing review has been submitted to the IRB for review and approval prior to the expiration date to extend the approval period;

Additional Notes: $\quad$ - Continuation with Amendment Expedited Approval per 45 CFR 46.110(b)(2) for New Funding: Minority Biomedical Research Support Program via NIH

IRB Approval has been provided for data analysis only. PI is to contact the IRB prior to the recruitment of additional subjects or further interactions/interventions with subjects.

Failure to comply with these conditions will result in withdrawal of this approval.

Please note that the IRB has the authority to observe, or have a third party observe, the consent process or the research itself. The Federal-wide Assurance (FWA) number for the Rutgers University IRB is FWA00003913; this number may be requested on funding applications or by collaborators.

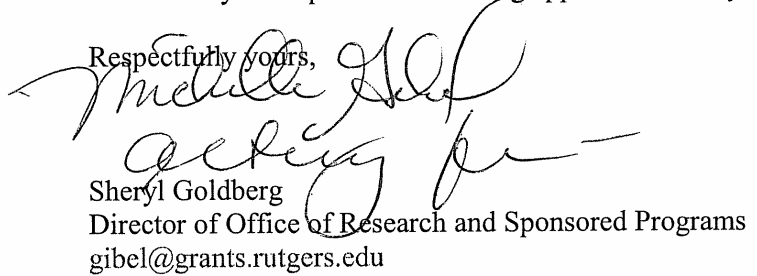

cc: Elise L. Lev 
Appendix B

Research Study Demographics

Jo Ann Cummings, Principal Researcher

Participant's Job Title

Are you a parent? YES NO

Participant's Age

Gender M F

Ethnic Origin

Years of Work Experience

Years of Pediatric Experience 


\section{Appendix C \\ Semi-Structured Interview Guide An Ethnography of the Culture of Pain in a Non-Pediatric Emergency Department}

1. Describe your role in the management of pediatric procedural pain?

2. Describe your main goals in treating procedural pain in the pediatric child.

3. The gold standard for measuring pain is asking the patient, but how do you determine a pediatric patient's intensity of pain before, during, and after procedures in a pre-operational child?

4. How do you make your decision about giving pharmacological treatments?

5. How do you make your decision about giving non-pharmacological treatments?

6. How does the frequency or timing of administration affect your decision about providing interventions? Are there certain times that work better or worse than others?

7. When you treat pain, how do you measure effectiveness? Do you feel that a pediatric patient should be in a pain free state? Why or Why not?

8. What do you identify as a problem in providing the pediatric patient in the emergency department with optimal pain relief?

9. As a healthcare provider in the emergency department you see and treat urgent and life-threatening situations, where does pediatric procedural pain rank on your list of competing priorities?

10. What do you believe is the general attitude about treating pediatric procedural pain in the emergency department?

11. What non-pharmacological and/or pharmacological actions are more commonly utilized by the staff when a pediatric patient requires a procedure?

12. What do you think is the value of non-pharmacological action(s) for pediatric procedural pain in the emergency department setting? How realistic is this type of treatment method?

13. What ethical or professional conflicts do you experience in managing the pediatric patient's procedural pain? 


\section{Appendix D}

\section{Informed Consent Form}

\section{An Ethnography of the Culture of Pain in a Non-Pediatric Emergency Department}

You are invited to participate in a research study that is being conducted by Jo Ann F. Cummings, who is a doctoral student in the Nursing Department at Rutgers University. The purpose of this research is to describe the unit culture of the non-pediatric emergency department, explore how healthcare providers evaluate and treat pediatric procedural pain and explore attitudes of healthcare providers towards treating pediatric procedural pain.

Participation in this study will involve: a brief demographic questionnaire, observations of nurses, physicians, and other health care providers in the assessment and management of pediatric procedural pain. During observations of these interactions notes will be made by the researcher. The researcher may ask to interview you with regard to the interactions that have been observed. A face to face semi-structured interview will be conducted which will last approximately 90 minutes. The interview will be audio taped with your permission and a copy of the transcript given to you to review and comment.

This research is confidential. The research records will include some information about you and this information will be stored in such a manner that some linkage between your identity and the response in the research exists. Some of the information collected about you includes your occupation, age, and gender. Please note that your name will be known to the researcher, but pseudonyms will be assigned to each individual. The researcher will keep this information confidential by limiting individual's access to the research data and keeping it in a secure location. Data will be stored securely in a key coded locked file cabinet and restricted access computer.

The research team and the Institutional Review Board at Rutgers University are the only parties that will be allowed to see the data, except as may be required by law. If a report of this study is published, or the results are presented at a professional conference, only group results will be stated. All study data will be kept for a length of time no greater than three years.

You have been told that the benefits of taking part in this study may be helpful to nurses and other healthcare providers' understanding how pediatric procedural pain is managed and perceived by healthcare providers in a non-pediatric emergency department unit. However, you may receive no direct benefit from taking part in this study.

Participation in this study is voluntary. You may choose not to participate, and you may withdraw at any time during the study procedures without penalty to you. In addition, you may choose not to answer any questions with which you are not comfortable.

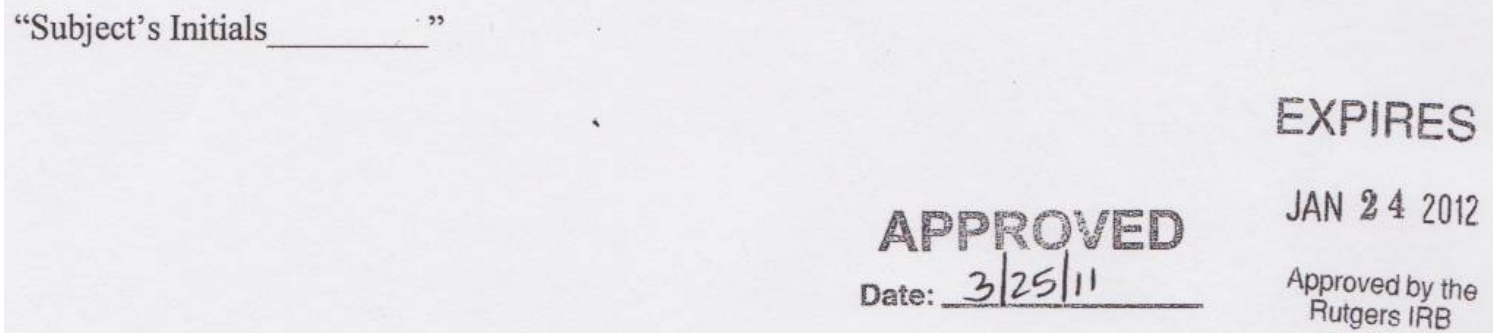




\section{Appendix D}

If you have any questions about the study or study procedures, you may contact myself, Jo Ann F. Cummings at 79 Bates Road Jackson, New Jersey 08527 or by phone (732) 363-1339 or (609) 503-2468 or email cummingsJP2003@yahoo.com

If you have any questions about your rights as a research subject, you may contact the IRB Administrator at Rutgers University at:

Rutgers University, the State University of New Jersey

Institutional Review Board for the Protection of Human Subjects

Office of Research and Sponsored Programs

3 Rutgers Plaza

New Brunswick, NJ 08901-8559

Tel: $732-932-0150$ ext. 2104

Email: humansubjects@orsp.rutgers.edu

You will be given a copy of this consent form for your records.

Sign below if you agree to participate in this research study:

\section{Subject (Print)}

Subject Signature Date

Principal Investigator (Print) Jo Ann F. Cummings, RN, MS, BCEN, PNP-C

Principal Investigator Signature Date 


\section{Appendix E}

\section{Informed Consent Form}

An Ethnography of the Culture of Pain in a Non-Pediatric Emergency Department

You are invited to participate in a research study that is being conducted by Jo Ann F.

Cummings, who is a doctoral student in the Nursing Department at Rutgers University. The purpose of this research is to describe the unit culture of the non-pediatric emergency department, explore how healthcare providers evaluate and treat pediatric procedural pain and explore attitudes of healthcare providers towards treating pediatric procedural pain.

Participation in this study will involve: observations of nurses, physicians, and other health care providers in the assessment and management of your child's procedural pain. During observations of these interactions notes will be made by the researcher. The researcher will have access to your child's medical record. With your permission, information collected from the chart will include documentation of procedural pain intensity scores, documentation about the effectiveness of pain-relieving interventions, documentation relating to the advice provided by the health care provider regarding procedural pain.

This research is confidential. The research records will include some information about your child such as age, gender, and procedure performed. This information will be stored in such a manner that no linkage between your child's identity and the response in the research exists. Please note that your name will be known to the researcher, but pseudonyms will be assigned to each individual. The researcher will keep this information confidential by limiting individual's access to the research data and keeping it in a secure location. Data will be stored securely in a key coded locked file cabinet and restricted access computer.

The research team and the Institutional Review Board at Rutgers University are the only parties that will be allowed to see the data, except as may be required by law. If a report of this study is published, or the results are presented at a professional conference, only group results will be stated. All study data will be kept for a length of time no greater than three years.

You have been told that the benefits of taking part in this study may be helpful to nurses and other healthcare providers' understanding how pediatric procedural pain is managed and perceived by healthcare providers in a non-pediatric emergency department unit. However, you may receive no direct benefit from taking part in this study.

Participation in this study is voluntary. You may choose not to participate, and you may withdraw at any time during the study procedures without penalty to you. In addition, you may choose not to answer any questions with which you are not comfortable.

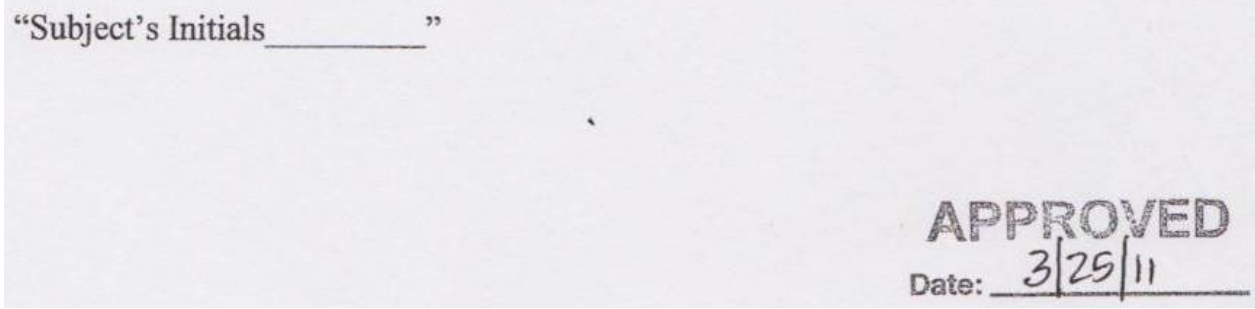

EXPIRES

JAN 242012 Date: $3\left[25111 \quad \begin{array}{c}\text { Approved by the } \\ \text { Rutgers IRB }\end{array}\right.$ 


\section{Appendix E}

If you have any questions about the study or study procedures, you may contact myself, Jo Ann F. Cummings at 79 Bates Road Jackson, New Jersey 08527 or by phone (732) 363-1339 or (609) 503-2468 or email cummingsJP2003@yahoo.com

If you have any questions about your rights as a research subject, you may contact the IRB Administrator at Rutgers University at:

Rutgers University, the State University of New Jersey

Institutional Review Board for the Protection of Human Subjects

Office of Research and Sponsored Programs

3 Rutgers Plaza

New Brunswick, NJ 08901-8559

Tel: $732-932-0150$ ext. 2104

Email: humansubjects@orsp.rutgers.edu

You will be given a copy of this consent form for your records.

Sign below if you agree to participate in this research study:

\section{Subject (Print)}

Subject Signature Date

\section{Principal Investigator (Print) Jo Ann F. Cummings, RN, MS, BCEN, PNP-C}

Principal Investigator Signature

Date 


\section{Appendix F}

Table 1: General Age Demographics in the Emergency Department

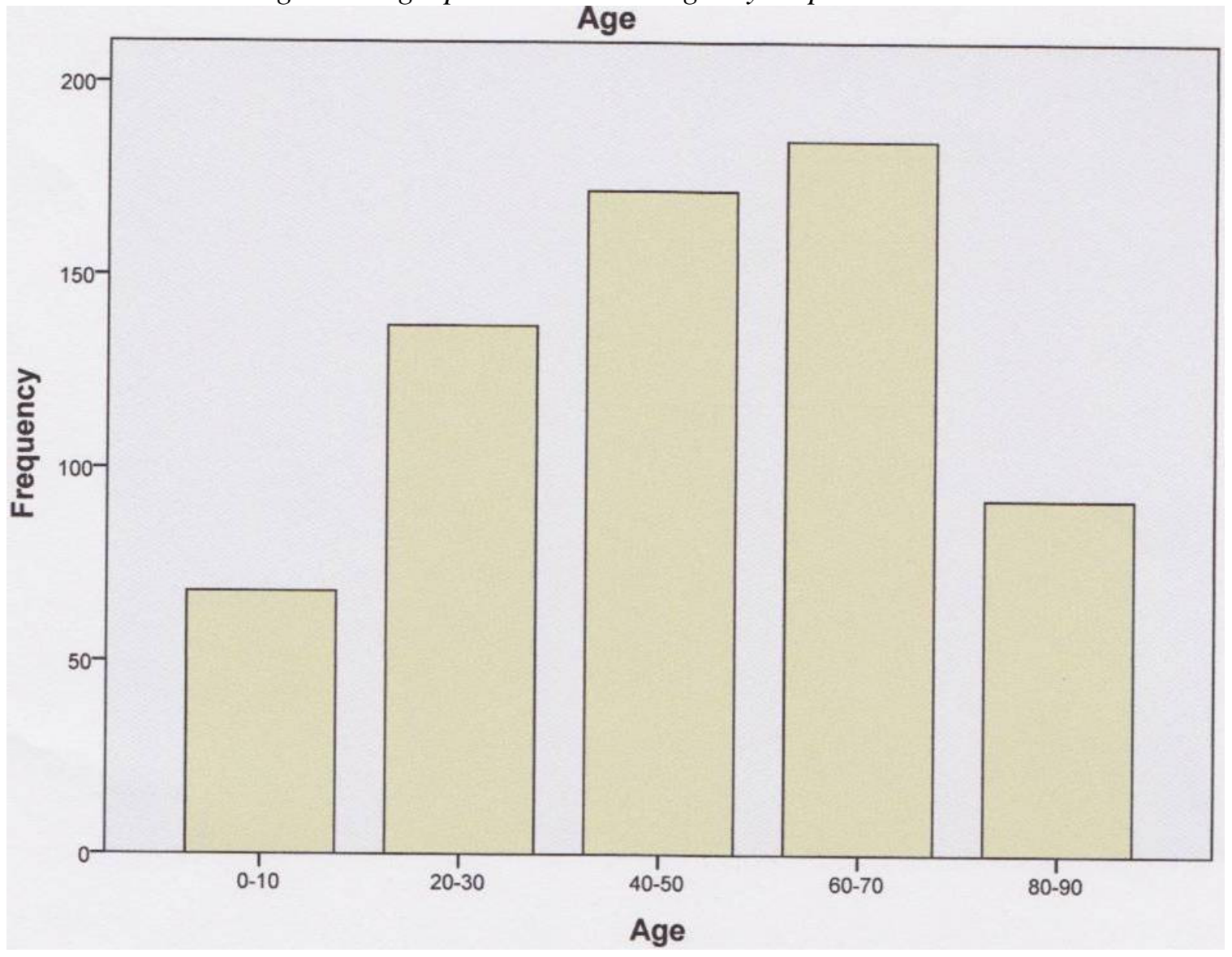




\section{Appendix G \\ Code Case Study}

APA=Addressing Patient Anxiety: The healthcare provider directs responses to the child's uneasiness of mind from illness, injury, or procedure.

$\mathrm{AP}=\mathrm{Apologetic:}$ a verbal expression offering regret, sorrow or empathy by the child caregiver or healthcare provider before, during, or after the procedure.

AS=Assessing The Individual: healthcare provider inquires as to the child's ability to handle the procedure.

$\mathrm{C}=$ Condescension: The act of patronizingly superior behavior or attitude

$\mathrm{CH}=$ Chatting: discussion of topics not related to current visit ( Walters, Prideaux, Worley, Greenhill, \& Rolfe, 2009)

$\mathrm{CL}=$ Control: the care provider allows the child to dominate or command the situation by providing choices.

$\mathrm{CR}=$ criticism: the act of passing judgment as to the merits of the child's behavior during a procedure.

$\mathrm{D}=$ Directional: to give direction to the parent or caregiver to perform a specific activity.

DI=Dismissive: The lack of interest or rejection to a child's verbalizations of pain, discomfort, or fear by the child care giver or healthcare provider.

DS= Demonstration Self: the healthcare provider performs the activity on themselves prior to the patient.

$\mathrm{G}=$ Positive reinforcement: the act of praise or reward to increase the rate of expected (good) behavior.

HT=History Taking: when the healthcare provider inquires about or the patient or parent of a child describes and details related to the chief complaint or prior illness (Walters, Prideaux, Worley, Greenhill, \& Rolfe, 2009).

I= Checking-In: behaviors used by care providers to make sure that patients are comfortable during a procedure (Abbe et al., 2007).

$\mathrm{K}=$ Education: presenting information regarding procedure, health, medical related task

L= Lie: a false statement. 


\section{Appendix G}

$\mathrm{M}=$ Medication: the use of pharmacology to assist in the alleviation of pain or anxiety of the child.

$\mathrm{NE}=$ Negotiation: the healthcare provider uses mutual discussion of the terms as to if and how a procedure is to take place.

NVD= Non-Visual Distraction: the use of non-visual tools (singing, counting, conversation) to re-direct the focus and attention of the child away from the current situation.

$\mathrm{P}=$ Procedure: any therapeutic or diagnostic procedure performed on the child.

$\mathrm{PC}=$ Physical comfort: basic interventions used to minimize pain and/or prevent the exacerbation of pain (ie, sling, ice pack).

$\mathrm{PP}=$ Parental Presence: the parents are present with their children when they undergo procedures.

PPI=Procedural Preparatory Information involves the depiction of the steps of the procedure, without descriptions of sensations (Zelter, Jay, \& Fisher, 1989)

PT=Play Therapy: The healthcare provider provides the child time to examine and play with medical/nursing equipment with the intent to relieve tension related to the current procedure or situation.

PN=Planning Treatment: The healthcare provider prescribes a medication, diagnostic, or treatment plan to be followed other than behavior change (Walters, Prideaux, Worley, Greenhill, \& Rolfe, 2009).

$\mathrm{Q}=$ Quick: performing an activity with speed and efficiency to completeness.

$\mathrm{R}=$ Restraint: to physically hold and stabilize the child in a position with or without the use of a specific device (ie drawer sheet, pillow case, or papoose). Can be either persons, equipment, or both.

$\mathrm{S}=$ Child Directed Speech: Speech patterns used by parents or caregivers when communicating with young children (i.e., simplified vocabulary, melodic pitch, change of intonation high in pitch.)

SPI = Sensory Preparatory Information: Sensory information provides a description of sensations (noises,smells, physical sensations) that a patient will experience during various points in the procedure (Zelter, Jay, \& Fisher, 1989).

$\mathrm{VD}=$ Visual Distraction: the use visual aids to re-direct the focus and attention of the child away from the current situation (ie favorite toy, I spy games, cartoons). 


\begin{abstract}
Appendix G
$\mathrm{V}=$ Advocacy: speaking on behalf of or in support of the child

$\mathrm{W}=$ Rewards: use of desirable incentive to increase (good) behavior.

$\mathrm{Y}=$ Playful Interaction: the health care provider is pleasantly humorous, jesting, or fun during the examination and/or care of the child.

$\mathrm{Z}=$ Reassurance: verbal or non-verbal (ie., the use of touch) responses by the caregiver to boost or promote psychological comfort in the child.
\end{abstract}

- $\quad \mathrm{E}=$ Eye contact: caregiver positions themselves to the eye-level of the child in order to build trust.

- $\mathrm{F}=$ Positioning: To position the child in a therapeutic manner that facilitates comfort during the procedure.

- $\mathrm{N}=$ Nickname: a descriptive name added or replacing the proper name of the child to show affection, or familiarity.

- $\mathrm{TO}=$ Touch: use of touch as a way to console the child during the procedure. 


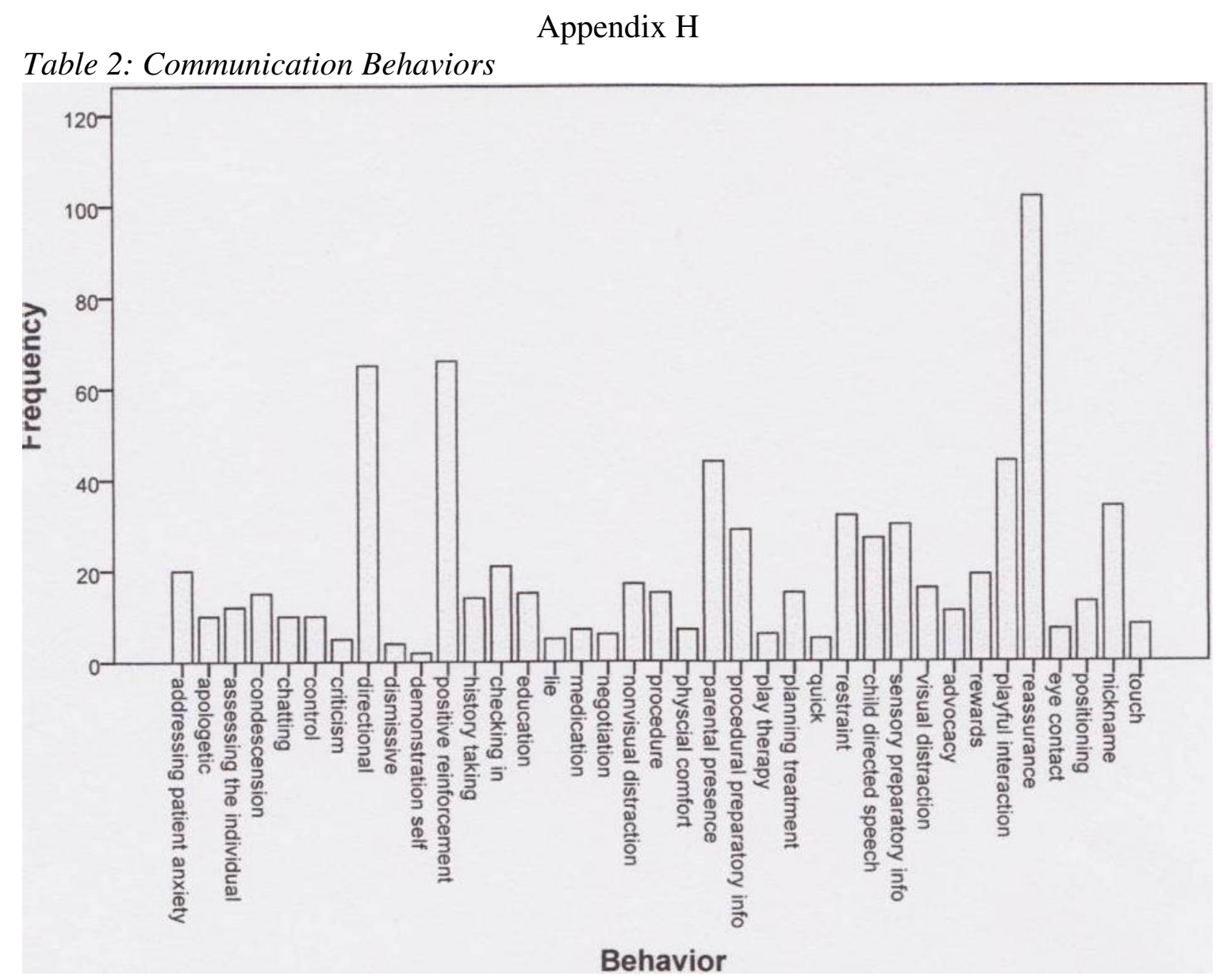


Appendix I

Table 3: Demographic Data Key Informants

\begin{tabular}{|c|c|c|c|c|c|c|}
\hline Key Informant & Ginelle & Maura & Kate Lyn & Lizza & Graham & Lain \\
\hline Position & Nurse & Nurse & Nurse & Nurse & Technician & Nurse \\
\hline $\begin{array}{l}\text { Parent: } \\
Y=\text { yes } \\
\mathrm{N}=\text { no } \\
\mathrm{NR}=\text { No response }\end{array}$ & $\mathrm{Y}$ & $\mathrm{Y}$ & $\mathrm{N}$ & $\mathrm{Y}$ & $\mathrm{N}$ & $\mathrm{Y}$ \\
\hline Age & 55 & 55 & 43 & 52 & 31 & 45 \\
\hline $\begin{array}{l}\text { Gender: } \\
\mathrm{F}=\text { female } \\
\mathrm{M}=\text { male }\end{array}$ & F & $\mathrm{F}$ & $\mathrm{F}$ & $\mathrm{F}$ & M & $\mathrm{F}$ \\
\hline Ethnicity & Hispanic & American & American & American & American & Hispanic \\
\hline $\begin{array}{l}\text { Years working } \\
\text { With children }\end{array}$ & 30 & 34 & 22 & 15 & 2 & 22 \\
\hline Years in profession & 12 & 34 & 22 & 30 & 2 & 22 \\
\hline
\end{tabular}




\section{Appendix $\mathbf{J}$ \\ Semi-Structured Interview Guide An Ethnography of the Culture of Pain in a Non-Pediatric Emergency Department}

\section{General Background}

1. How long have you been a nurse?

2. What areas have you worked prior to or other than the emergency department?

3 . Tell me about your experiences as a nurse working with children in pain.

4. Does having your children of your own affect how you approach and manage a young child's pain?

5. How comfortable are you with performing invasive procedures on young children?

6 . How do think knowledge base affects procedural pain management in pediatric patients?

\section{Role}

7. What are the more common experiences you've had with pediatric procedural pain in the emergency department setting?

8. When I say pediatric procedural pain, what are the things that come to your mind?

9. Who performs the more common procedures blood work, IV's on young children? Sutures?

10. Describe your role in the management of pediatric procedural pain?

11. How much control do you have over treating pediatric procedural pain?

\section{Evaluation}

12. From your experience, describe for me how you know a young pediatric patient has pain?

13. What scales, if any, are used to assess pain in young pediatric patients? How reliable do you believe the scale is? Do you believe it is just pain? Why? What do you believe is a better indicator of a young child's pain? Do you use the scales to measure procedural pain? If not, when do you use the scales?

14. The gold standard for measuring pain is asking the patient, but how do you determine a pediatric patient's intensity of pain before, during, and after procedures in a pre-operational child (in a child who does not understand the scales)?

15. What resources, if any, are available to treat pediatric procedural pain?

\section{Value}

16. What is your goal when managing procedural pain in young pediatric patients? 
17. How important is a young pediatric patient's comfort during a procedure? What do you do? Why?

18. Do you believe that the amount of pain experienced by a young child during a procedure has any negative long term effects?

19. Describe your main goals in treating procedural pain in the pediatric child.

20. How do you make your decision about giving pharmacological treatments? Do you use nurse driven protocols for managing pediatric procedural pain? If not, do you think nurse driven protocols would be appropriate for managing pediatric procedural pain?

21. What non-pharmacological interventions, if any, do you use more commonly when treating procedural pain in a young pediatric patient?

22. What value is non-pharmacological interventions for the treatment of pediatric procedural pain in a young pediatric patient?

23. How do you decide what approach to take with a young child for a procedure?

24. Does the frequency or timing of an intervention affect your decision about providing interventions? Are there certain times that work better or worse than others?

25. Do you feel that a pediatric patient should be in a pain free state? Why or Why not?

26. What would you identify as a problem in providing the pediatric patient in the emergency department with optimal pain relief?

\section{Issues and Concerns}

27. How much of a priority is pain control in young children when you have to perform an invasive procedure in the emergency department?

28. Do you believe you are providing the best nursing care when managing pediatric procedural pain in young children?

29. What do you believe is the general attitude about treating pediatric procedural pain in the emergency department?

30. What non-pharmacological and/or pharmacological actions are more commonly utilized by the staff when a pediatric patient requires a procedure?

31. What ethical or professional conflicts have you experienced in managing the pediatric patient's procedural pain?

32. What concerns do you have about providing pain management for pediatric procedures in the emergency department?

33. Are there any other issues that come to mind when you think about procedural pain management in the young pediatric patient? 
Appendix K

\section{AUDIO ADDENDUM TO CONSENT FORM}

You have already agreed to participate in a research study entitiled: An Ethnography of the Culture of Pain in the Non-Pediatric Emergency Department conducted by Jo Ann F. Cummings. I am asking for your permission to allow audiotape (sound) as part of that research study. You do not have to agree to be recorded in order to participate in the main part of the study.

The recording(s) will be used for analysis by the principal researcher. The recordings will include your occupation, gender, age, and work experiences. The recording(s) will be stored in a locked file cabinet and linked with a code to subjects' identity; and will be retained for no greater than three years when the recording(s) will be destroyed.

Your signature on this form grants the investigator named about permission to record you as described about during participation in the above-referenced study. The investigator will not use the recording(s) for any other reason than that/those stated in the consent form without your written permission.

Subject (Print)

Subject Signature Date

Prinicipal Investigator Jo Ann F. Cummings, RN, MS, BCEN, PNP-C

Principal Investigator Signature

Date 


\section{MEMO: ED PAIN POLICY}

\section{Appendix L}

PURPOSE: Provision of pain treatment protocols for the adult and pediatric non-urgent patient.

SCOPE: All Emergency Department physician and nursing staff

TEXT: Non-pharmacologic protocol

- Cold elevation for musculoskeletal and soft tissue injuries $<72$ hours old

- Immobilization of suspected fractures in position of comfort using slings and splinting

- Cleansing and dressing

- Distractions as adjunct to pain interventions such as relaxation techniques, reading materials, games, or television

Non-urgent pain protocol for adults (i.e. toothache, earache, contusions, lacerations, minor burns, musculoskeletal pain)

- Obtain vital signs and allergies

- Administer Motrin $400 \mathrm{mg}$ po if not taken within 4 hours prior to arrival (PTA) and not contraindicated (see below)

- If Motrin taken less than 4 hours PTA and pain level greater than 4 administer Percocet (oxycodone hydrochloride) $5 \mathrm{mg} / 325 \mathrm{mg}$ one tablet po

- Repeat pain assessment and vital signs one hour post Percocet administration

- Should a patient choose to leave, after receiving pain medication, but before provider examination, all efforts should be made to involve the provider and consideration should be given to completion of an "Against Medical Advice" form

- Patient must have a designated driver if given Percocet.

Non-urgent pain protocol for pediatric patients

- Obtain vital signs and allergies

- Administer Tylenol (acetaminophen) $15 \mathrm{mg} / \mathrm{kg}$ po if not taken within 4 hours PTA

- If Tylenol taken less than 4 hours PTA and pain level greater than 4 , administer Motrin $10 \mathrm{mg} / \mathrm{kg}$ po

- If obvious skeletal deformity,

1. children ages 3-6 years old administer Tylenol with codeine elixir $5 \mathrm{ml}$ po

2. children ages 7-10 years old administer Tylenol with codeine elixir $10 \mathrm{ml}$ po

- Repeat pain assessment and vitals signs after one hour

- If pain level greater than 4, one hour after Tylenol or Tylenol with Codeine administration, consult physician

CONTRAINDICATIONS TO ANALGESICS

- Allergy or sensitivity to the medication 
- Liver or kidney conditions/ failure

- Hemodynamic instability

- Chest pain or abdominal pain

- Multisystem trauma

- Pregnancy; consult physician

- Gastric disease

- Coumadin (warfarin)

- Altered level of consciousness

- Intoxication or suspected substance abuse (acute)

- Lung disease with poor oxygenation/aeration 


\section{MEMO: ED PAIN POLICY (PISCES)}

SUBJECT: Extremity injury protocols (PISCES APPROACH)

PURPOSE: To promote timely pain management and comfort measure of Emergency

Department patients with extremity injuries by initiating a diagnostic and therapeutic plan by the RN.

POLICY: The RN may implement a standing order for $\mathrm{x}$-rays and pain medication for the simple extremity injury. This may include soft tissue injuries, fractures, and strains/sprains.

\section{OBJECTIVES:}

- To promote a timely evaluation of the Emergency Department patients presenting with a simple extremity injuries

- To rapidly address the need for pain medication and comfort measures

- To insure the highest quality of care to the extremity injured patient

\section{UTILIZATION:}

- Communicate to all appropriate personnel

- Serve as a reference guide for the appropriate application of the policy by the RN who has been educated on the procedure.

\section{PROCEDURE:}

- Patients presenting to triage with the complaint of an extremity injury will be triaged as per policy.

- Pain assessment should be completed and pain medication offered*

- Documentation should include: subjective complaints (i.e. numbness, tingling), objective assessment Pallor, Pulse distal to injury, Paralysis, Paresthesia (sensation to light touch or pinprick)

*Inform physician immediately if assessment indicates neurovascular compromise, crush injury, or suspected foreign body.

Pregnancy status should be ascertained and urine HCG completed as needed

- Immobilize and apply ice to the injured part

- Remove jewelry from injured extremity and give to family

- Sling should be applied for upper body extremity injuries

- Comfort/compassion should be assressed

- Elevate the injured part, if possible

- Place extremity in position of comfort

- Show concern and empathy

Expedite radiographic evaluation

- Order should be completed by triage nurse

- Patient taken to x-ray by MST or radiology technician 
- Patient should be returned to treatment area and advised to wait for provider evaluation

\section{RESPONSIBLE PERSONS}

- Emergency Department RN for accurate assessment of the patient, initiation of pain medication and appropriate $\mathrm{x}$-ray request

- Emergency Department provider for assuring the quality of care given to patients presenting with extremity injuries

\section{GUIDELINES FOR PAIN MANAGEMENT}

- Either of the following medications may be given based upon patient preference, review of medication allergies and past medical history

Ibuprofen:

Dose: $10 \mathrm{mg} / \mathrm{kg}$ up to $400 \mathrm{mg}$

Contraindications: ASA/NSAID induced asthma, history of GI bleed, caution if elderly, CHF, ACE inhibitors, anticoagulants

Acetaminophen:

Dose: $15 \mathrm{mg} / \mathrm{kg}$ infants and children; $650 \mathrm{mg}$ for adults

Contraindications: Allergy to Tylenol. Caution if impaired liver function, chronic ETOH usage, impaired renal function, G6PD deficiency or PKU 
MEMO: Triage assessment protocol

\section{SUBJECT: EMERGENCY DEPARTMENT TRIAGE AND ASSESSMENT}

Purpose: Provision of guidelines for initial assessments and acuity determinations on all arriving patients so that:

1. medical attention and treatment are given first to those with the most serious illnesses and injuries, and

2. presenting patients are matched with appropriate resources.

Scope: All providers and nursing staff.

Policy Statement: Every arriving patient will receive a timely brief assessment by an experienced registered nurse. The assessment will be focused on chief compliant and presenting subjective and objective findings to determine priorities of care. Nurse triage does not constitute medical screening examination. Neither triage nor registration will delay medical screening examination or treatment when there is a qualified medical provider available.

Procedure:

1. Triage will occur with the first RN-patient interaction.

2. Patients arriving by ambulance as well as those arriving ambulatory will be triaged by an $\mathrm{RN}$ in conjunction with quick registration.

3. Registration will take place at bedside or in triage area with consideration of patient acuity and bed availability. Registration will not delay medical screening or any emergent necessary treatment.

4. A rapid, focused triage assessment will take place soon after patient arrival and will include:

A. Subjective and objective findings

B. History limited to its relevance to the immediate episode

C. ESI (Emergency Severity Index) triage acuity level (1-5)

D. If a question arises regarding acuity level, the triage nurse will consult the E.D. Physician/Provider.

5. When a bed or treatment space is available for any arriving patient, the patient will be taken directly to that bed/space without delay. Triage may take place before or after provider evaluation depending on department volume and patient flow.

6. Regardless of department volume and available treatment space. Level 1 patients will be taken directly to the treatment area for physician evaluation and treatment.

7. Rapid triage will be done on levels 2-5 patients and as deemed necessary, emergent patients will be taken directly to the treatment area for provider evaluation and treatment.

8. Pulse, respirations and pulse oximetry will be taken during triage on all patients. Blood pressure will be taken dependent on presentation as necessary.

9. During the hours of 11a-9p a medical screening exam will be performed on patients in the waiting area by a mid-level provider in order of the direction of a collaborating physician in the ED. 
10. Standing orders may be initiated by the triage RN according to policy ES 555 .

11. Patients will not be triaged out or otherwise referred or diverted to a physician's office or any other healthcare setting without medical screening examinations and stabilization of emergency medical conditions.

12. The Triage nurse will maintain communication with the Charge Nurse/ED Coordinator with regards to bed/treatment space availability.

13. Initiation of approved standing order sets will not delay triage of subsequent arriving patients but will be handed off to an accepting RN.

14. The Triage Nurse will document and report the Charge Nurse and/or Primary Nurse assuming the care of any patient on whom standing orders or protocols have been initiated during triage.

15. The Triage Nurse, Charge Nurse, ED coordinator, or Service Representative will update patients in the waiting room concerning delays and wait times.

16. Patients who must be placed in the waiting room will be reassessed by the Triage Nurse based on needs and acuities. Reassessments will be documented.

17. In the rare instance that PICIS is unavailable, the nurse will document using Triage Form 55555 and enter the information into the computer when PICIS is again available. 
MEMO: Hospital to open Pediatric Emergency Department

The Trentonian

June 17, 2011 06:37 PM EST

For a child to go to an emergency department in many respects is a scary situation and so by providing this softer environment we will be able to deliver the care as well as be able to a have them feel that they're in a much friendlier environment. It also allows us to clinically integrate better with our friends at RWJ in New Brunswick a with the BMSCH a so will be able to provide a seamless level of care for children as they continue to move forward.

Rainy weather is an indication of providing a clean new slate for the development of this new pediatric services wing that we are creating here there will be 8 new patient rooms for pediatric patients as well as 8 new additional fast track rooms. The total project is a $\$ 5$ million project and we will be breaking ground and begin to implement the project by the end of October the beginning of November.

I: And is this to service the increase demand for this a....

$\mathrm{R}$ : There is extraordinary increased demand for this service as we have looked at things and we are certainly cognizant of that as we have done our analysis and what have you so we feel that we'll be able to do this and provide a great deal more of accessibility as well collaboration with the RWJ in NB at the BMSCH

I: Does this increase mean there's going to be an increase of physicians?

R: Yes there will be specifically we will be increasing the number of physicians in the emergency department but they will be specifically pediatric physicians as well as an increase in the nursing staff all of whom will have been trained in pediatric medicine so that will be of a great benefit to the community, when you add up the additional staff its about 20 new people who will come on board.

\section{Memo:}

My thoughts on this impromptu interview with the newspaper reporter: The CEO conveyed his support for the new project but pointed out the difference of pediatric environment to be "this softer and friendlier environment". What the CEO describes as softer and friendlier is an important concept to the psychosocial care of developing children, meeting the physical and psychosocial demands of developing early humans. The care of children is more than just slightly different with smaller equipment. Young children are not smaller versions of developed adults. 


\section{Appendix M}

MEMO: Thoughts first day

$03 / 27 / 2011$

$14: 30$

The emergency department walk-in started out very slow with no people in the waiting area. The time frame from nothing to everything went quickly, people started to walk in at a regular rate. The staff appeared to change their pace when patients complaining of chest pain presented themselves, or patients who did not appear well from the outer triage nurse's visible assessment upon entering the waiting area. During these times the registrar, the outer triage nurse, and the nurse in the private conference room simultaneously "worked" on the patient and moved the person through the process at a quick pace assessing, registering, and working up the patient within minutes of arrival.

It was very rare that I noticed a young child in the emergency department waiting area. The first child I documented seeing was when I first arrived at 10:59 am. The child appeared to be a pre-teen waiting to be seen. The next child I documented seeing was the pre-school aged little girl leaving with her father at 13:18 PM. I have been told by the nurse educator that children tend to come in more in the evening time especially during the week. The nurse educator recommended that I should start out observing around the peak times when pediatric patients come into the emergency department to make the most of my observations.

I am not sure how the triage system is structured at this emergency department there appears to be many steps in the process. My questions:

What goes on in the private conference room?

What goes on in the triage booth? How do they decide who goes into the booth? Does everyone? If not, what happens to the people once they are assessed by the outer triage nurse?

I rarely saw a child in the 2.5 hours of observation. I think that I will start to schedule my observation times based on when the nurse educator believes the children are most likely to utilize the emergency department services. 
MEMO: Observation Easter Sunday "Guilt" and the research process $04 / 24 / 2011$

$23: 30$

I knew that I was going into the hospital today; I was hoping that with some schools on Spring Break and returning to school on Monday and after a beautiful day that children would have flooded the emergency department with all types of injuries. The weather has been labile cold or rainy or both and today was one of the nicest hot days we have had. For my own children who are 5 and 7 years of age even leaving after a day of being together was a bit upsetting. I prepared their Easter baskets with my husband the night before, and I made a big Easter meal for lunch. The kids played outside while I cleaned the house and slept for the evening's events. I usually host a family meal, but I wanted to conserve my energy for field work. My children start to prepare for bed at $6 \mathrm{PM}$ and are in bed by 7 PM but when I was preparing to leave for the hospital my daughter started to cry. "Why do you have to go? Please don't!" I handed my emotional daughter to my husband, but he said, "I'm not going to comfort her for something so silly. I don't want to reinforce this behavior." I tried to console her, but I also had to get ready I said, "I will be right back. You will go to sleep, and when you wake up I will be home. I always kiss you when I get home after I have cleaned up. It will be okay." I don't know if it helped, but as I was leaving my brother and his wife showed up, and that offered and needed distraction. I said good night and left. I found out later that even with my husband, brother, and sister-in-law here she was crying. My sister-in law found my daughter red faced and teary eyed when she asked her what was wrong she told her, "I miss mommy."

Even coming home at night is a well thought out plan before I leave I have to move all my bath and facial products to the outside hallway sometimes I get home before 2am or $1 \mathrm{am}$ it depends on the evening and how many children are seen toward the closing hours of prompt care. When I come home I move about quietly, I quietly shut the door, I turn down all the lights, and I go straight to the hallway bathroom and start to clean up. If I am lucky, no one wakes up and I slide into bed unnoticed.

I was a little put off by the fact that my computer code did not work that meant I had to ask one of the staff to look at their notes each time a child came in, and that is cumbersome. Plus if the staff are busy I will not interrupt their work for my gains.

Today was steady stream of patients. It appears that a majority of the pediatric patients come into the prompt care area. Nurse Ja is helpful when I came in she said there is a 3 year old with peanut allergy and wheezing but there was a 2 year old coming in with right leg pain. It is difficult to split myself when there are pediatric patients coming in from different areas of the emergency department. I have to be able to follow the patients throughout their treatment. Not all of the nurses or physicians in the emergency department have consented to let me observe.

I think that having read that last email I don't want to approach anyone of the ED staff at least not tonight. I felt guarded I didn't want to talk just write and keep to myself. It didn't help that my IBEX login and password did not work. I had to ask Nurse Ja if I 
could look at her charting, and that was only good for one or two times during the slower or "low volume" periods.

Dr G. is nice she asked me about what my study was for I shared with her it is for my dissertation she asked me what I wanted to prove and I said nothing I'm not here to prove just to observe how it is I shared with her the statistics $30 \%$ of children go to generalized ED's not specialized but no documentation on what happens and how those providers treat children. She shared with me how she used to take her kids to St P. and how it was so hard to find the ED.

I don't understand why they are so dependent or quick to delegate responsibility to the tech when they could do it. Why did the nurse delegate the splint to the tech. Why didn't she do it? It would have been good times to bond with the family reassess the patient condition. I didn't see the nurse check the splint on the leg to make sure it was right. I understand teaching and doing but the tech was inundated with requests.

The people seem more cordial moving in and out saying hi or cracking smiles instead of just stares.

It kind of bothered me when the emergency department director saw I was here and didn't make any attempt to introduce or say hello to me. I have to say I have been staying away. I have a feeling she doesn't want me here maybe it's a turf issue not getting her permission and being sprung with the IRB approval of the study. The ED director is a petite woman maybe 50's. She looks very worn, wrinkled skin, Caucasian, deep voice almost like a smoker, stringy frizzy hair blonde long. She's wearing heels so when she walks you can hear her coming. She looks worn I don't know another word to better describe her.

Dr. G offered me some of the Easter food the staff brought but I cordially declined. I would like to think it is a step toward acceptance but Dr G is different she's very professional and motherly. The PA asked about how many patients or how many hours I thought that was an interesting question. I explained that when I leave and expand the notes then everything is coded the I look through the coding to see if the data is saturated until then I keep coming back.

Note:

I'm starting to see patterns and I would like to compare findings against interviews with staff I will start to schedule interviews more regularly. 


\section{MEMO: GENERAL COMMENTS}

$05 / 07 / 2011$

20:05

A few days ago I decided to keep my distance from the emergency department. My last day here I had to manage 4 children at the same time, going in and out of the rooms while treatments were being provided, a majority of that time I listened to the children crying and fighting. I think that the last 2 pediatric cases with the children were the most difficult the screaming, the fighting. For me, it feels unnatural to stand there and do nothing. It goes against my own maternal instincts and my professional duty as a nurse. It was stressful and frustrating. It is distressing; it is the worst feeling of helplessness on my part. I need to take the time to step back from the field for just a moment to keep from being burned out. So I took time to step away. I also think the staff started asking many questions of me, my background, and who I was as an individual. I don't want to get comfortable; I don't want to lose that critical analytical edge. I needed to be away from here.

Today, the downtime of observing versus the excitement of seeing a potential candidate: when I sit looking for patient on the board I look to see how long they've been here, I check to see for what the nurse rated the child. A 4-year old is in the emergency department, but has been in there for over a hour and a half. From the hallway I could see a child in a bed, resting a curled up, I thought the activity with all the blood draws and IV sticks was all done and it was more of a waiting game. The pain for this child was 5 using the numeric scale $0-10$. I thought this was interesting that the pain rating of children's varies dependent upon the nurse or is it the child? Could a 4 year old truly identify pain, understand the concept of rating pain using the numeric rating scale? Does every nurse have their preference of a scale? Do they stick to using one scale every time they chart? Whose pain intensity rating is this, the nurses, the parents, the patients?

On days that there are low volumes patients are brought back and greeted by the tech and the nurse simultaneously. Usually the tech is the first to take a temperature. The temperature tympanic was removed from the front triage area because of its questionable readings; this was noted in the staff meeting minutes from the March write- up. 


\section{MEMO: REMARKS}

$09 / 05 / 2011$

In healthcare we don not have the luxury to cherry pick our patients that means that each individual who enters through our front door is accepted and treated. We accept them with their varied conditions, social situation, genetic predispositions, and psychopersonality variability.

So why is it that healthcare provider are so quick to talk about the variability of children during painful procedures. During some informal conversations with the emergency department staff discussion of children and procedures elicited this common response, "some are so good, but some are so bad. It is so variable and dependent on the child". One physician was so bold to state, "When you are done with you circuitous analysis you will find that it is child dependent".

So, why the one size fits all care? Why not tailor care to that child?

I have found that an underlying shared basic assumption is that these healthcare providers work in an EMERGENCY setting where timing of treatment and maintaining the flow of patients is key to an emergency department's basic function. Accountability and focus of priorities in this institution is set highly on patient satisfaction, but how many pre-verbal and pre-operational children are sent patient satisfaction surveys. Keeping this principle in mind then HCP tend to gear their care toward how the parent viewed the visit, NOT the child. In this case, if parents viewed being seen in a quick manner as the most important thing then that is what is addressed.

The one size fits all approach means that regardless of the patient each patient is treated or managed for procedures in a similar manner. ED HCP "negotiate with parents to accommodate them to the ED's needs be efficient, get it done safely, and move on to the next patient.

I believe that Culture is the main theme, the sub-culture of the emergency department learning from them how they care for children during procedures.

\section{Culture is the main theme}

Desensitization:

Frequency of procedure

\&/or distance less likely to address

Underlying assumptions

Resilient

Bounce back
Doesn't need to be addressed: erroneous procedure, downplay

$\underline{\text { Tenuous Relationships }}$

$\underline{\text { Fragmented Care }}$ 
Schein discusses how organizational culture is set by those in leadership positions who drive the basic values, beliefs, and actions of those involved with the service.

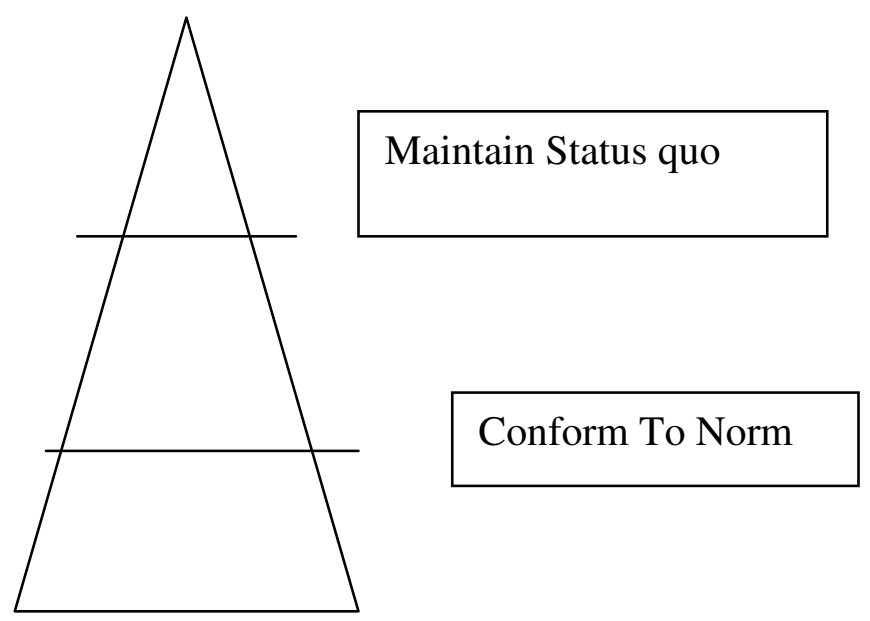

\section{$\underline{\text { ED Values }}$}

- Maintain flow Avoid overcrowding Avoid Backing up

- Timely treatment

- Prioritize Identify and treat those of greatest need

- Patient satisfaction (number one uncompromising commitment toward patient satisfaction. A philosophy that touches every aspect of the hospital)

They work to maintain the ED's most basic function.

How things are documented are influenced by distal factors??

How things are performed are influenced by both distal and proximal??

Regardless of an individual's beliefs the values of the ED took precedence in shaping the practice of the HCP.

Questions of interest, why not invest?

- Human resource issue

- Financial issue

Look at healthcare management books on ED organization.

Existing models 
HCP Characteristics + Setting

First level prescriptive theory I/C
System Variables: Tanabe

$$
\text { Treatmemt }
$$

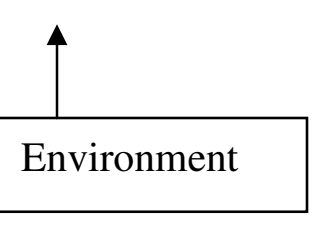

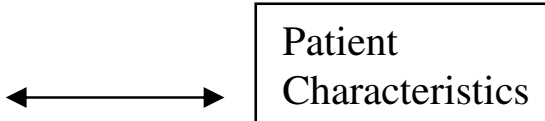

Attitude/Belief

\section{4}

Characteristics

Cultural Negotiation and Accomodation working with families to manage pediatric procedural pain.

ED Negotiate

Family Accommodate

Potential result of ED basic underlying values?

Leininger 


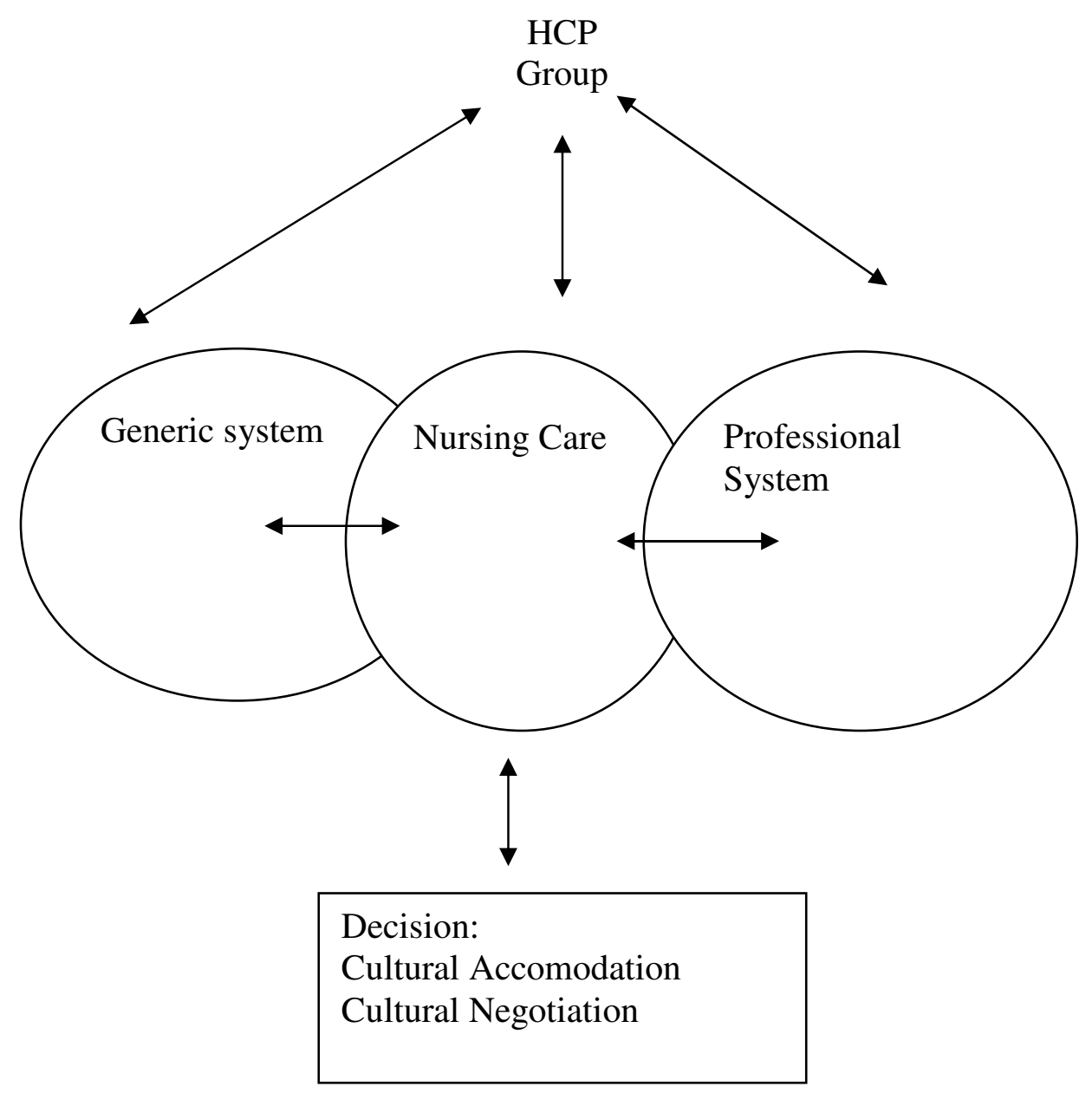

What is the end result? The end result cultural negotiation-Accomodation

Systems approach to bigger picture

Culture Care Action Based Decision

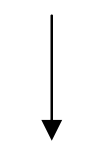

Comfort Care theory for interventions

What about hierarchy? Status power relationships

If I refer to the triangle at the top represents the leadership, ED medical director (legitimate power, leadership, defines culture), Nurses and ancillary staff ( powerlessness, conformity, subordination)

The lower the level the greater the powerlessness and subordination.

Power and compliance $=$ social influence peers request 
Power and conformity= majority vs. individual

510 Fiske Conceptual Definition: Social Influence: Broadly encompasses any changes in beliefs, attitude, or behavior that result from interpersonal interaction.

Social influence typically refers to interpersonal processes that change other people's behavior.

Fiske specific definition social influence: how the thoughts, feelings, and behaviors of individuals are influenced by the actual, imagined, or implied presence of others.

Operational Definition

Compliance as social influence form a peer's request (526) social influence $=$ compliance

Final thoughts

Cultural Negotiation Accomodation

Social Influence

Compliance

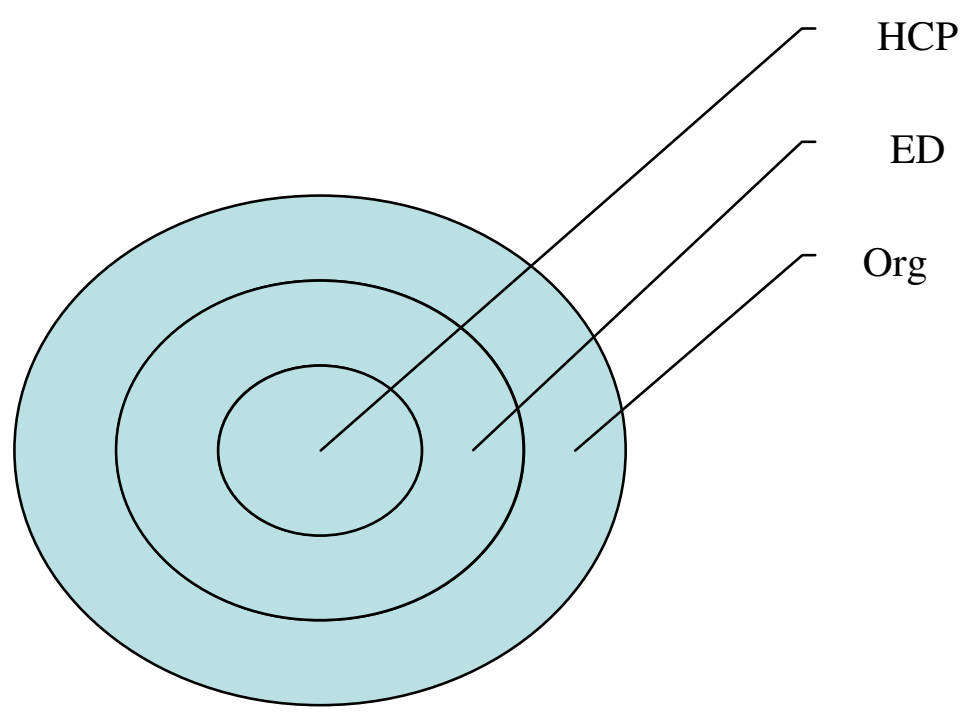


Appendix N

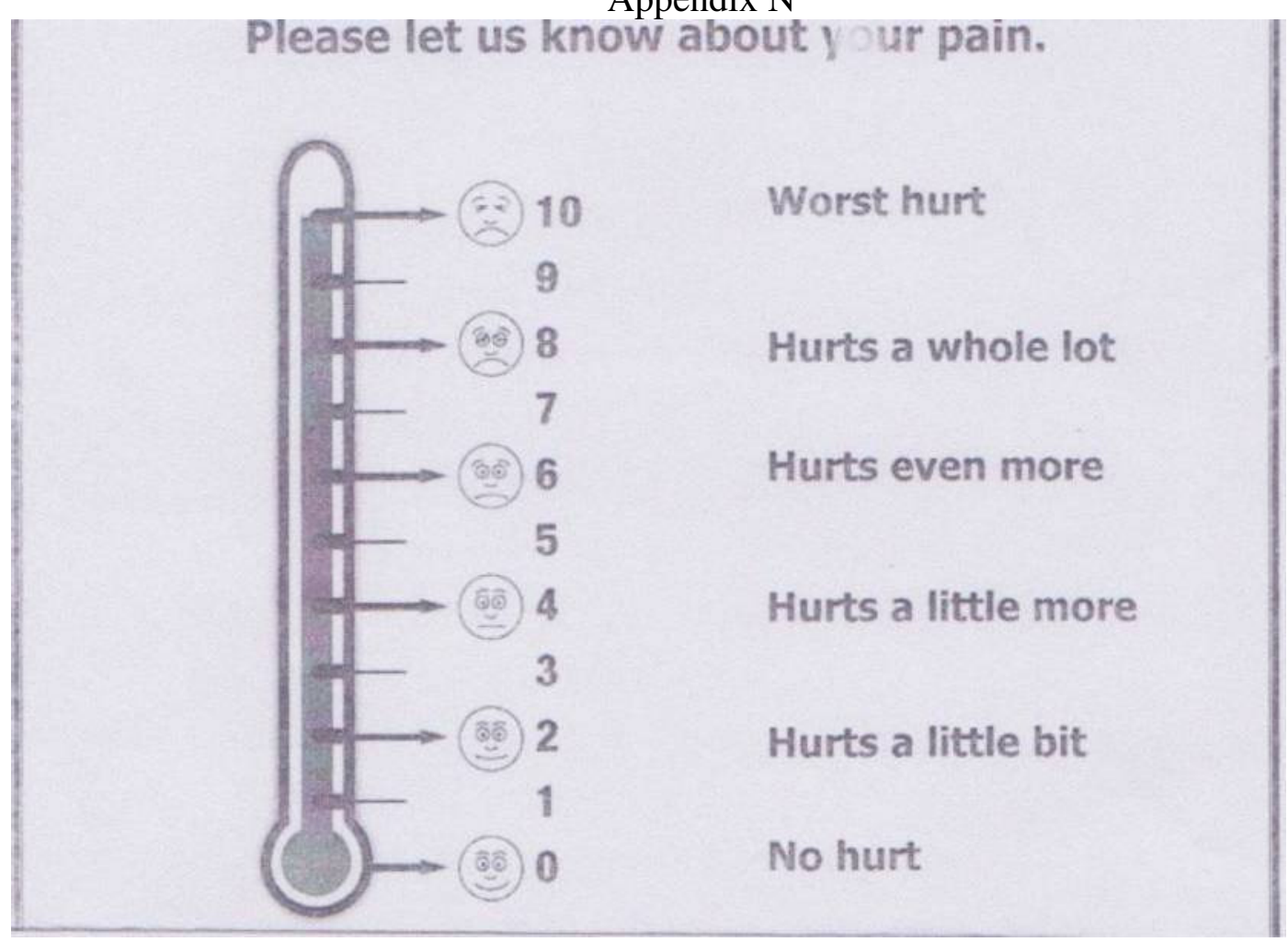




\section{Appendix $\mathrm{O}$}

Restraint Policy

A restraint is defined as any manual method of physically restricting a person's freedom of movement, or normal access to his or her body, material, or equipment, attached or adjacent to the patient's body that he/she cannot easily remove.

Devices that have the effect of restricting a patient's freedom of movement and cannot be easily removed by the patient is considered to be a restraint.

Restraints shall be used after all practical alternatives have been employed. Restraints shall be used based on observed behaviors to either support medical-surgical healing or to protect the patient from injury to self or others.Every attempt should be made to notify family/significant other and include them in the care plan decision. restraints may not be used for discipline or punish a patient or for convenience of staff.

Emergency restraints may be initiated under the direction of the registered nurse to directly support medical healing. The physician shall be notified and an appropriate order shall be otained within one hour. Orders must be time limited and may not exceed 24 hours. the type of restraint and reason shall be specified in the physician's orders. Each episode of restraint use is documented in the medical record. Document restrant occurrences on the restraint flow sheet found in the when ordering restraints for a behavioral health reason, a face to face evaluation must be completed by a physician within 1 hour of the initiation of the restraint. 


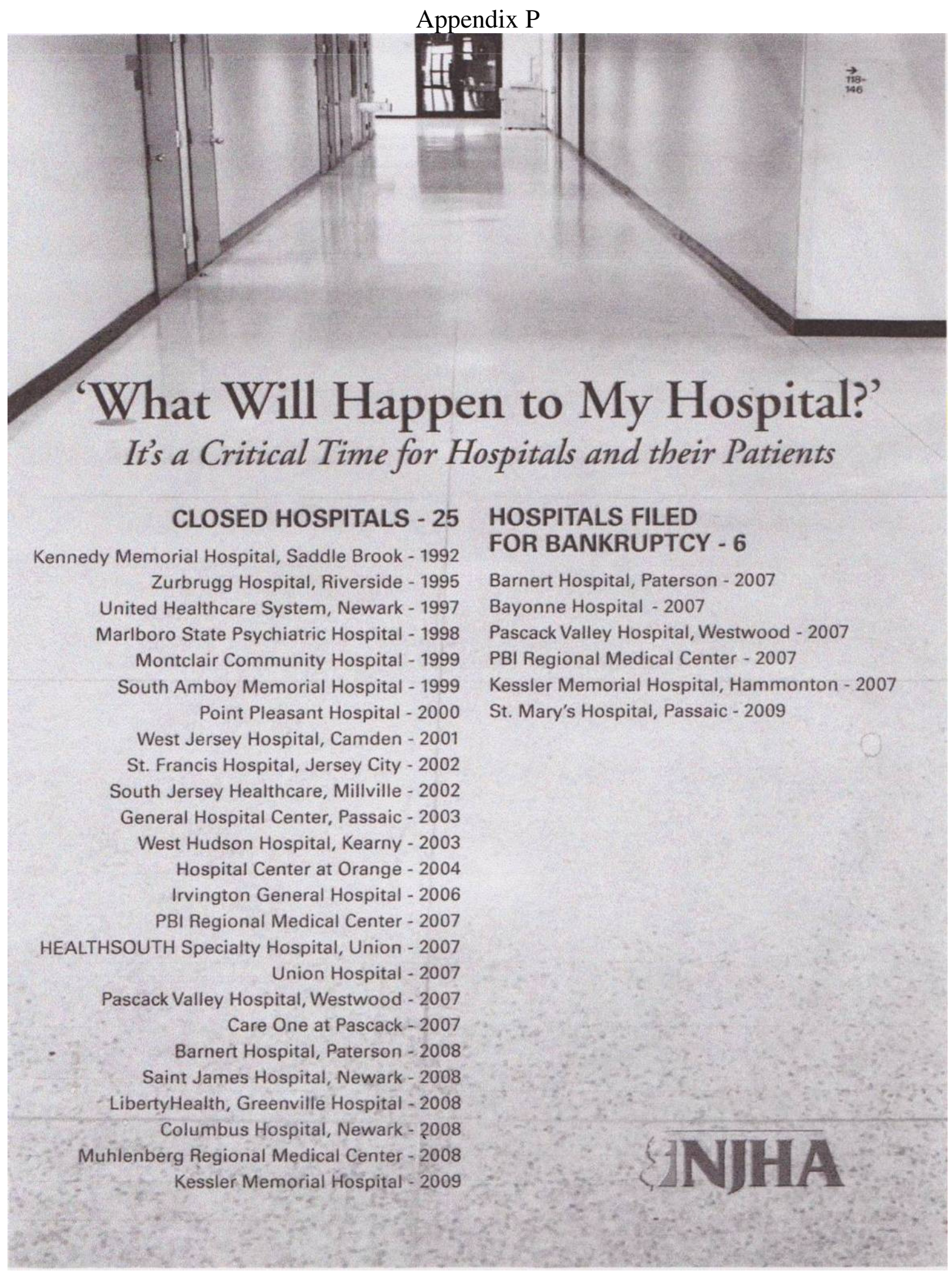




\section{Appendix Q \\ SUBJECT: Management of the Patient with Pain}

Purpose: To assure comprehensive assessment, reassessment, and management of pain through various pain management techniques that are consistent with the scope of care, treatment, services, and patient condition.

To respect and support the patient's right to pain management.

Scope: This policy applies to inpatients, ED patients, the Family Health Center, outpatients undergoing (a) treatment of physical/ occupational therapy or (b) an invasive/interventional procedure.

\section{Text: A. Patient Rights}

The hospital recognizes and supports the adequate management of pain as a patient right. The goal of pain management is to improve the quality of life of patients experiencing pain by providing relief for those in acute and chronic pain through assessment, reassessment, and pain management techniques.

Upon admission, the registration clerk will provide the patient a statement of Patient Rights. The patient will be informed of their rights as they relate to pain management in this document.

\section{B. Assessment}

1) Pain Levels Definition: The Likert, FLACC, Wong-Baker, NIPS pain assessment tools will be utilized as appropriate to the patient's condition to yield a numeric pain score. The pain score will used to determine the level of pain as follows:

Mild Pain- pain score of 1,2,3

Moderate Pain- pain score of 4,5,6

Severe Pain-pain score of 7,8,9,10

The pain level will be utilized in determining administration of pharmaceutical treatment when orders are present based on level of pain.

2) Pain Scales: Fellowship uses methods to assess pain that are consistent with the patient's age, condition and ability to understand which include the Likert numeric scale, FLACC, Wong-Baker, and NIPS scale.

\section{3) Inpatient}

Upon admission to the hospital, nursing staff will assess for the presence of pain. When pain is present, the admission assessment will include: the location/site of pain, pain intensity, pain duration/frequency, characteristics, aggravating factors, alleviating factors and intervention. 
4) Outpatient

Upon admission to the outpatient area, the presence of pain will be assessed. When pain is present the admission assessment will include the location/site of pain, pain intensity, pain duration/frequency, characteristics, aggravating factors, alleviating factors and intervention.

C) Re-Assessment

1. Inpatient: Reassessment of pain will be conducted at least every shift and before and after pain medication administration in a time frame appropriate to the medication and route. Reassessment includes: location/site of pain, pain intensity, acceptable comfort level, description of pain, interventions, response to interventions, and additional interventions, Additional information related to comfort can be documented on the Interdisciplinary Progress Notes.

2. Outpatient: Reassessment of pain will be conducted as appropriate to the reason the patient is presenting for care or services and before and after pain medication administration in a time frame appropriate to the medication and route. Re-assessment will include at least pain intensity, and response to interventions.

D) Plan of Care

1. Inpatient: Upon assessment, if "pain" is identified as a problem, pain will be incorporated in the patient's Interdisciplinary Plan of Care in accordance with policy (NUR-A-5555-Z). Plan of care will include the patient/family when possible and consider alternative treatment modalities, spiritual/cultural, and life cycle needs of the patient as appropriate. When indicated, the Licensed Independent Practitioner will be notified of alterations in pain or inadequate pain relief from ordered interventions.

2. Outpatient: Healthcare providers will consider any treatments or procedures for their potential to cause pain and plan care appropriately. Plan of care will include the patient/family when possible and consider alternative treatment modalities, spiritual/cultural and life cycle needs of the patient as appropriate. When indicated, the Licensed Independent Practitioner will be notified of alterations in pain or inadequate pain relief from ordered interventions.

\section{E). Patient/Family Education}

Based on the patient's condition and assessed needs, the education and training provided to the patient includes discussion of pain, the risk for pain, the importance of effective pain management, the pain assessment process, and methods for pain management.

F) Discharge Planning

Upon discharge, a discussion with the patient/family on relieving interventions used for pain such as medications and alternate modalities should take place. 


\section{G) Staff Education}

Information regarding the hospital pain management program is provided during departmental orientation for specific disciplines. Ongoing education based on staff needs, physician, patient and family feedback, performance improvement initiatives or policy and procedure changes will be provided for staff as needed. 


\author{
Curriculum Vitae \\ Jo Ann F. Cummings \\ Lakewood, New Jersey \\ Born June 07
}

Education

1992 Diploma Monsignor Donovan High School, Toms River, NJ

1996 Bachelor of Science- Rutgers, the State University of New Jersey CON

1999 Master of Science- Rutgers University Graduate School Newark, NJ

2006 Post Master Certificate Education Rutgers University CON, NJ

2006 Certificate Distance Education Thomas Edison State College, NJ

2012 Doctor of Philosophy in Nursing Research Rutgers Graduate School Newark, NJ

Employment

2011 Graduate Assistant Rutgers University MBRS Program Grant \# R25 60825-06, NJ

2006-Present RN Educator Meridian Health System, NJ

2002- 2004 Injury Prevention Program Coordinator RWJUH, NJ

2001-2002 Advanced Practice Nurse/Clinical Nurse Specialist LMHS, FL

2000-2001 Pediatric Nurse Practitioner Private Practice, NJ

1999-2001 Clinical Instructor Rutgers University College of Nursing, NJ

1999-2001 Registered Nurse PICU/ED NEI, NJ

1996-2001 Registered Nurse Emergency Services KMC, NJ

Cummings, J.F. (2006, July 24). Being Green: A case of pediatric narrow complex tachycardia. Advance for Nurses, 8(16), 31 